\title{
Algoritmos assíncronos \\ de iteração de política para \\ processos de decisão markovianos \\ com probabilidades intervalares
}

Willy Arthur Silva Reis

\author{
DISSERTAÇÃO APRESENTADA \\ $\mathrm{AO}$ \\ Instituto De MatemÁticA E EstatísticA \\ DA \\ Universidade DE SÃo PAUlo \\ PARA \\ OBTENÇÃO DO TÍTULO \\ DE \\ Mestre em CiênCIAS \\ Programa: Ciência da Computação \\ Orientadora: Profa. Dra. Karina Valdivia Delgado
}

Durante o desenvolvimento deste trabalho o autor recebeu auxílio financeiro do CNPq

São Paulo, agosto de 2019 


\section{Algoritmos assíncronos \\ de iteração de política para \\ processos de decisão markovianos \\ com probabilidades intervalares}

Esta versão da dissertação contém as correções e alterações sugeridas pela Comissão Julgadora durante a defesa da versão original do trabalho, realizada em 02/08/2019. Uma cópia da versão original está disponível no

Instituto de Matemática e Estatística da Universidade de São Paulo.

Comissão Julgadora:

- Prof $^{\mathrm{a}}$. Dr ${ }^{\mathrm{a}}$. Karina Valdivia Delgado (orientadora) - EACH-USP

- Prof ${ }^{a}$. Dr ${ }^{\mathrm{a}}$. Anna Helena Reali Costa - POLI-USP

- Prof. Dr. Reinaldo Augusto Costa Bianchi - FEI 


\section{Agradecimentos}

À Professora Karina pela orientação, pelas oportunidades de realização deste trabalho e de monitorias, pelos ensinamentos, por toda a ajuda durante estes anos e pela paciência.

À professora Leliane pelas dicas valiosas e pelo trabalho conjunto nos meus primeiros artigos publicados.

Ao professor Valdinei pelo trabalho conjunto e pelas ideias valiosas para pesquisas futuras.

Aos professores do IME, da EACH e da POLI que contribuíram com minha formação neste mestrado.

Agradeço aos meus familiares, sempre se importando comigo e com o meu trabalho, e principalmente aos meus pais, Maria e José Geraldo, por todo apoio durante este processo, e ao meu irmão Luiz.

Agradeço à Rubia, minha companheira, pelo amor e pelo incentivo em trilhar este caminho.

Agradeço aos meus amigos do LIAMF, em especial a Erika, o Felipe, o Ignasi, a Julissa, o Milton, a Paula, a Viviane, e ao Daniel, não só irmão de orientação como amigo de muitos gostos em comum.

Agradeço aos meus amigos do futebol do IME, por compartilhar o tempo e o gosto nessa atividade tão essencial.

Este trabalho não teria sido possível sem o apoio do CNPq (processo número 134336/2016-0). 


\section{Resumo}

Um Processo de Decisão Markoviano (MDP) pode ser usado para modelar problemas de decisão sequencial. No entanto, podem existir limitações na obtenção de probabilidades para modelagem da transição de estados ou falta de confiabilidade nas informações existentes sobre estas probabilidades. Um modelo menos restritivo e que pode resolver este problema é o Processo de Decisão Markoviano com Probabilidades Intervalares (BMDP), que permite a representação imprecisa das probabilidades de transição de estados e raciocínio sobre uma solução robusta. Para resolver BMDPs de horizonte infinito, existem os algoritmos síncronos de Iteração de Valor Intervalar e Iteração de Política Robusto, que são ineficientes quando o tamanho do espaço de estados é grande. Neste trabalho são propostos algoritmos assíncronos de Iteração de Política baseados no particionamento do espaço de estados em subconjuntos aleatórios (Robust Asynchronous Policy Iteration - RAPI) ou em componentes fortemente conexos (Robust Topological Policy Iteration - RTPI). Também são propostas formas de inicializar a função valor e a política dos algoritmos, de forma a melhorar a convergência destes. $\mathrm{O}$ desempenho dos algoritmos propostos é avaliado em comparação com o algoritmo de Iteração de Política Robusto para BMDPs para domínios de planejamento existentes e um novo domínio proposto. Os resultados dos experimentos realizados mostram que (i) quanto mais estruturado é o domínio, melhor é o desempenho do algoritmo RTPI; (ii) o uso de computação paralela no algoritmo RAPI possui um pequeno ganho computacional em relação à sua versão sequencial; e (iii) uma boa inicialização da função valor e política pode impactar positivamente o tempo de convergência dos algoritmos.

Palavras-chave: Planejamento Probabilístico, Processos de Decisão Markovianos com Probabilidades Imprecisas, Iteração de Política Assíncrono. 


\section{Abstract}

A Markov Decision Process (MDP) can be used to model sequential decision problems. However, there may be limitations in obtaining probabilities for state transition modeling or lack of reliability in existing information on these probabilities. A less restrictive model that can solve this problem is the Bounded-parameter Markov Decision Process (BMDP), which allows the imprecise representation of the transition probabilities and reasoning about a robust solution. To solve infinite horizon BMDPs, there are synchronous algorithms such as Interval Value Iteration and Robust Policy Iteration, which are inefficient for large state spaces. In this work, we propose new asynchronous Policy Iteration algorithms based on state space partitioning in random subsets (Robust Asynchronous Policy Iteration - RAPI) or in strongly connected components (Robust Topological Policy Iteration - RTPI). We also propose ways to initialize the value function and policy of the algorithms, in order to improve their convergence. The performance of the proposed algorithms is evaluated in comparison with the Robust Policy Iteration algorithm for BMDPs for existing planning domains and a proposed new domain. The results of the experiments show that (i) the more structured the domain, the better is the performance of the RTPI algorithm; (ii) the use of parallel computing in the RAPI algorithm has a small computational gain compared to its sequential version; and (iii) a good initialization of the value function and policy can positively impact the convergence time of the algorithms.

Keywords: Probabilistic Planning, Markov Decision Processes with Imprecise Probabilities, Asynchronous Policy Iteration. 


\section{Sumário}

$\begin{array}{lc}\text { Lista de Abreviaturas } & \text { ix }\end{array}$

Lista de Símbolos $\quad$ xi

Lista de Figuras $\quad$ XV

1 Introdução $\quad 1$

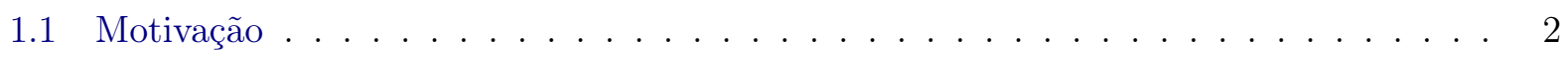

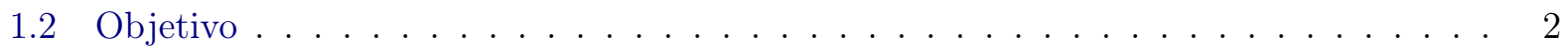

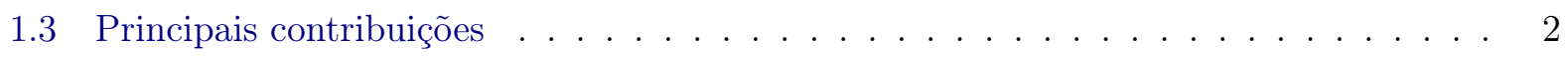

1.4 Organização . . . . . . . . . . . . . . . . . . . . . . . 2

2 Processos de Decisão Markovianos $\quad 3$

2.1 Definição de MDP . . . . . . . . . . . . . . . . . . . . . . . . . . 3

2.2 Algoritmos para MDPs . . . . . . . . . . . . . . . . . . . . 6

2.2.1 Algoritmos síncronos baseados em programação dinâmica . . . . . . . . . 6

2.2.2 Algoritmos assíncronos baseados em programação dinâmica . . . . . . . . . . 7

2.2 .3 Programação linear . . . . . . . . . . . . . . . . . . . . 10

2.3 MDP Fatorado . . . . . . . . . . . . . . . . . . . . . . . . 11

3 Processos de Decisão Markovianos com Probabilidades Imprecisas 13

3.1 MDP-IP: Definição . . . . . . . . . . . . . . . . . . . . . . . 13

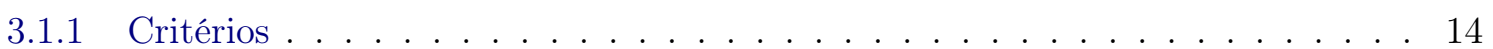

3.1 .2 Critério Robusto . . . . . . . . . . . . . . . . 15

3.2 Algoritmos . . . . . . . . . . . . . . . . . . . . 16

3.2 .1 Iteração de Política para MDP-IP . . . . . . . . . . . . . . . . 16

3.2 .2 Iteração de Valor para MDP-IP . . . . . . . . . . . . . . . . . 16

3.2 .3 Programação matemática . . . . . . . . . . . . . . . . . . 17

3.3 MDP-IP Fatorado . . . . . . . . . . . . . . . . . . . . . . . 17

3.4 Outros modelos - Subclasses de MDP-IP . . . . . . . . . . . . . . . . 18

4 Processos de Decisão Markovianos com Probabilidades Intervalares 21

4.1 BMDP: Definição . . . . . . . . . . . . . . . . . . . . . . 21

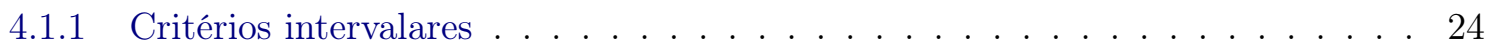

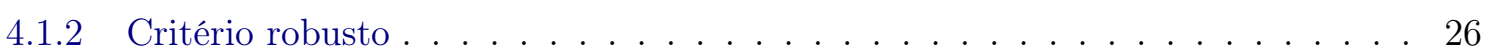

4.2 Algoritmos . . . . . . . . . . . . . . . . . . . 26 
4.2 .1 Cálculo da função Q para BMDPs . . . . . . . . . . . . . . . . 26

4.2 .2 Iteração de Valor Intervalar ～. . . . . . . . . . . . . . . . . . . . 27

4.2 .3 Iteração de Política Robusto . . . . . . . . . . . . . . . . . . . . . . . . 28

5 Programação assíncrona para BMDPs $\quad 31$

5.1 Operadores de atualização conservativos robustos . . . . . . . . . . . . . . . . . 31

5.2 Política Inicial e Função Valor Inicial . . . . . . . . . . . . . . . . . . . . . . . . 33

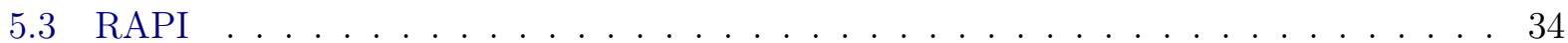

5.4 Iteração de Política Topológico Robusto . . . . . . . . . . . . . . . . . . . . 37

5.4.1 Componentes fortemente conexos de um BMDP . . . . . . . . . . . . . . . . 37

5.4 .2 Ordem de atualização ótima . . . . . . . . . . . . . . . . . . 37

5.4 .3 Algoritmo RTPI . . . . . . . . . . . . . . . . . . . 37

6 Resultados Experimentais $\quad 39$

6.1 Domínios de planejamento . . . . . . . . . . . . . . . . . . . . . 39

6.2 RAPI-sequential vs RAPI-parallel . . . . . . . . . . . . . . . . . . . 41

6.3 Comparando RPI, RAPI* e RTPI . . . . . . . . . . . . . . . . . . . . . . 43

7 Trabalhos relacionados $\quad 47$

7.1 Outros horizontes para BMDPs . . . . . . . . . . . . . . . . . 47

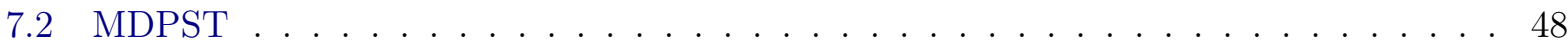

7.3 Outros modelos com probabilidades imprecisas e incerteza . . . . . . . . . . . . 48

7.3.1 Cadeias de Markov Imprecisas . . . . . . . . . . . . . . . . . . . . . . . 48

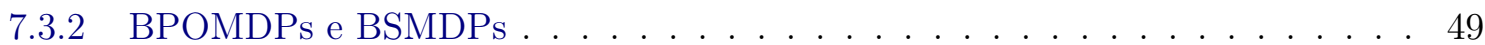

7.3 .3 MDPs Possibilísticos . . . . . . . . . . . . . . . . . . . . . . 49

8 Considerações Finais $\quad 51$

8.1 Resumo das contribuições . . . . . . . . . . . . . . . . . . . . . . 51

8.2 Publicações geradas . . . . . . . . . . . . . . . . . . . . 52

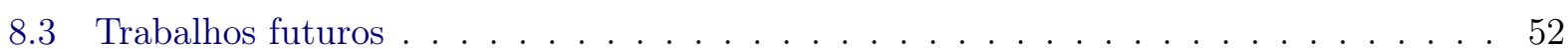

Referências Bibliográficas $\quad 53$ 


\title{
Lista de Abreviaturas
}

\author{
ADD Diagrama de Decisão Algébrico (Algebraic Decision Diagram) \\ API Iteração de Política Assíncrono (Asynchronous Policy Iteration) \\ BMDP Processo de Decisão Markoviano com Probabilidades Intervalares (Bounded- \\ Parameter Markov Decision Process) \\ BPOMDP Processo de Decisão Markoviano Parcialmente Observável com Probabilidades \\ Intervalares (Bounded-Parameter Partially Observable Markov Decision Process) \\ BSMDP Processo de Decisão Semi Markoviano com Probabilidades Intervalares (Bounded- \\ Parameter Semi Markov Decision Process) \\ BSSP BMDP de Caminho Estocástico Mínimo (Bounded-Parameter Stochastic-Shortest \\ Path) \\ CPT Tabela de Probabilidade Condicional (Conditional Probability Table) \\ DAG Grafo Acíclico Dirigido (Directed Acyclic Graph) \\ DBN Redes Bayesianas Dinâmica (Dynamic Bayesian Network) \\ DCN Rede Credal Dinâmica (Dynamic Credal Network) \\ DET Planejamento Determinístico (Deterministic Planning) \\ GMDP Processo de Decisão Markoviano Direcionado a Meta (Goal-Directed Markov De- \\ cision Process) \\ IVI Iteração de Valor Intervalar (Interval Value Iteration) \\ $\mathrm{IVI}_{\text {opt }} \quad$ Iteração de Valor Intervalar Otimista (Optimistic Interval Value Iteration) \\ $\mathrm{IVI}_{\text {pes }} \quad$ Iteração de Valor Intervalar Pessimista (Pessimistic Interval Value Iteration) \\ MDP Processo de Decisão Markoviano (Markov Decision Process) \\ MDP-IP Processo de Decisão Markoviano com Probabilidades Imprecisas (Markov Decision \\ Process with Imprecise Probabilities) \\ MDPST Processo de Decisão Markoviano com Transição Valorada por Conjuntos (Marko- \\ vian Decision Process with Set-valued Transition) \\ MPI Iteração de Política Modificado (Modified Policy Iteration) \\ NON-DET Planejamento não Determinístico (Non-Deterministic Planning) \\ PADD Diagrama de Decisão Algébrico Parametrizado (Parametrized Algebraic Decision \\ Diagram) \\ PI Iteração de Política (Policy Iteration) \\ RAPI Iteração de Política Assíncrono Robusto (Robust Asynchronous Policy Iteration) \\ RPI Iteração de Política Robusto (Robust Policy Iteration) \\ RTPI Iteração de Política Topológico Robusto (Robust Topological Policy Iteration) \\ SCC Componente Fortemente Conexo (Strongly Connected Component)
}


SPST Problema do Caminho Estocástico Mínimo com Transição Valorada por Conjuntos (Stochastic Shortest Path with Set-valued Transition)

SPUDD Planejamento Estocástico Utilizando Diagramas de Decisão (Stochatic Planning Using Decision Diagrams)

SSP Caminho Estocástico Mais Curto (Stochastic Shortest Path)

SSP-MDP Caminho Estocástico Mais Curto (Stochastic Shortest Problem Markov decision Process)

TVI Iteração de Valor Topológico (Topological Value Iteration)

VI Iteração de Valor (Value Iteration) 


\section{Lista de Símbolos}

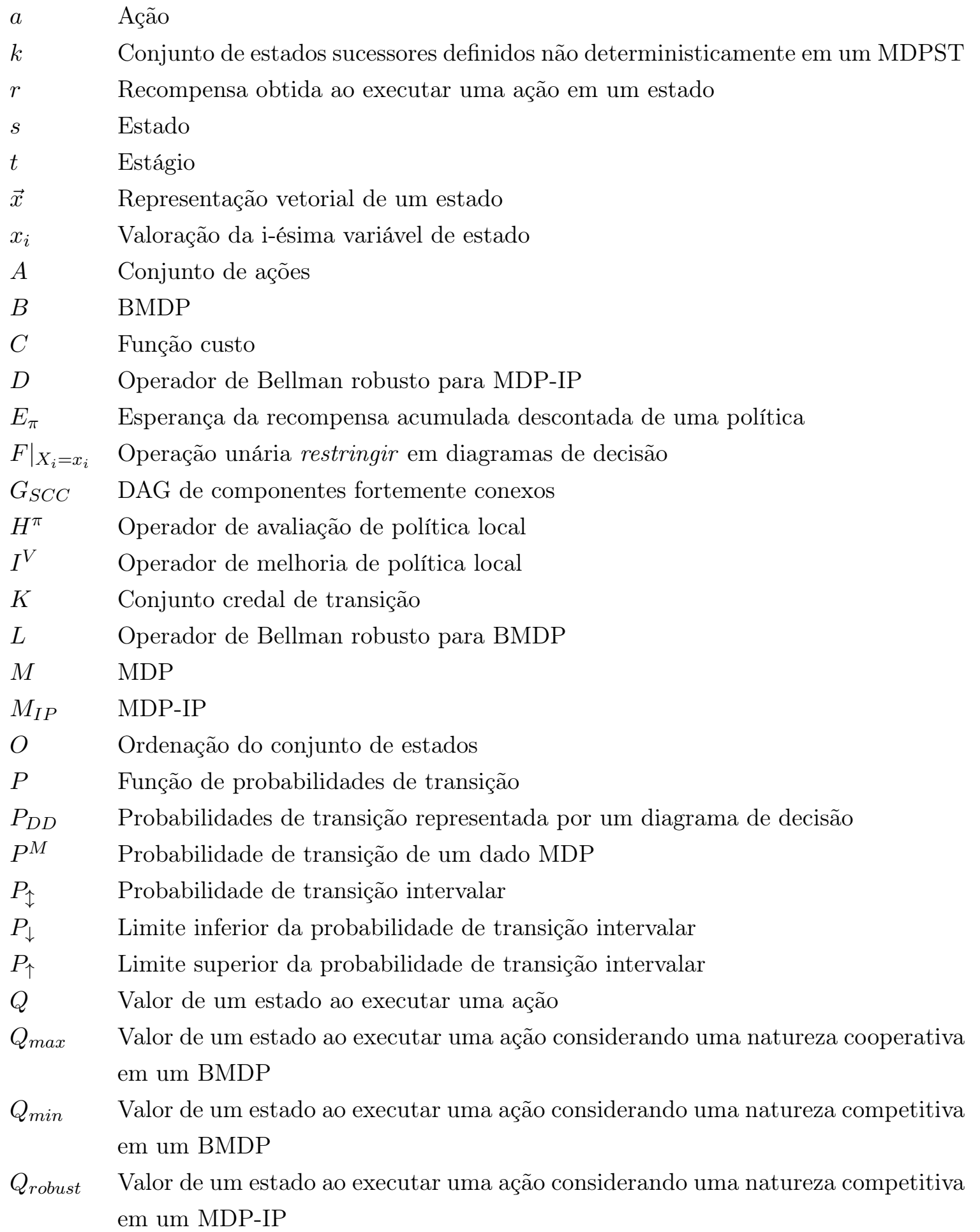


$Q^{*} \quad$ Valor ótimo de um estado ao executar uma ação

$R \quad$ Função recompensa

$R_{D D} \quad$ Função recompensa representada por um diagrama de decisão

$R_{\uparrow} \quad$ Função recompensa intervalar

$R_{\downarrow} \quad$ Limite inferior da função recompensa intervalar

$R_{\uparrow} \quad$ Limite superior da função recompensa intervalar

$S \quad$ Conjunto de estados

$S_{i} \quad$ Subconjunto do conjunto de estados

$T \quad$ Operador de Bellman para MDP

$T_{\text {opt }} \quad$ Operador de Bellman otimista para BMDP

$T_{\text {pes }} \quad$ Operador de Bellman pessimista para BMDP

$V \quad$ Função valor

$V^{0} \quad$ Função valor inicial

$V^{t} \quad$ Função valor no estágio t

$V^{\pi} \quad$ Função valor de uma política

$V^{\pi, M} \quad$ Função valor de uma política para um dado MDP

$V^{*} \quad$ Função valor ótima

$\underline{V} \quad$ Limite inferior da função valor

$\bar{V} \quad$ Limite superior da função valor

$V_{D D} \quad$ Função valor representada por um diagrama de decisão

$V_{\uparrow} \quad$ Função valor intervalar

$V_{\downarrow} \quad$ Limite inferior da função valor intervalar

$V_{\uparrow} \quad$ Limite superior da função valor intervalar

$V_{\uparrow}^{\text {opt }} \quad$ Função valor ótima otimista

$V_{\uparrow}^{\text {pes }} \quad$ Função valor ótima pessimista

$V_{\uparrow}^{\pi} \quad$ Função valor intervalar de uma da política

$V_{\downarrow}^{\pi} \quad$ Limite inferior da função valor intervalar de uma da política

$V_{\uparrow}^{\pi} \quad$ Limite superior da função valor intervalar de uma da política

$X \quad$ Conjunto de variáveis de estado

$X_{M_{\uparrow}} \quad$ Conjunto de MDPs de maximização de ordem

$X_{i} \quad$ i-ésima variável de estado

$Z \quad$ Partição do conjunto de estados

$\alpha \quad$ Número de estados por subconjunto

$\gamma \quad$ Fator de desconto

$\epsilon \quad$ Erro máximo

$\pi \quad$ Política

$\pi^{*} \quad$ Política ótima

$\pi^{0} \quad$ Política inicial

$\pi^{t} \quad$ Política no estágio t

$\rho^{V_{\uparrow}^{t}} \quad$ Conjunto de ações que produzem o valor máximo do limite superior

$\sigma_{\downarrow}^{V_{\downarrow}^{t}} \quad$ Conjunto de ações que produzem o valor máximo do limite inferior

$\sum_{x_{i}} F \quad$ Operação de marginalização em diagramas de decisão

$\varphi \quad$ Conjunto de restrições lineares 
$\leq_{o p t} \quad$ Comparador de intervalos utilizando o critério otimista

$\leq_{\text {pes }} \quad$ Comparador de intervalos utilizando o critério pessimista

$\ominus \quad$ Operação subtração para diagramas de decisão

$\oplus \quad$ Operação adição para diagramas de decisão

$\otimes \quad$ Operação multiplicação para diagramas de decisão 


\section{Lista de Figuras}

2.1 Exemplo do espaço de estados, ações e transições de um MDP. . . . . . . . . . . . . 4

2.2 Contraexemplo de Williams e Baird [WB93] . . . . . . . . . . . . . . . . . 8

3.1 Exemplo de conjunto credal representado pela região cinza [DSdB11a] . . . . . . . . . 14

3.2 Exemplo de MDP-IP. a) Espaço de estados, ações e transições de um MDP-IP. b) Conjunto de restrições lineares $\varphi_{i}$ referentes aos conjuntos credais de transição. . . 15

3.3 Relações entre diferentes modelos usados em planejamento probabilístico. . . . . . . 19

4.1 Exemplo do espaço de estados de um BMDP. As probabilidade de transição são rotuladas com intervalos, por exemplo, $[0.2,0.5]$ (o intervalo $[1.0,1.0]$ indica uma transição determinística). . . . . . . . . . . . . . . . . . . . . . . . . . . . . . . . . 22

4.2 Exemplo de MDP de maximização de ordem. . . . . . . . . . . . . . . . . 23

4.3 Exemplo de BMDP e sua função valor intervalar para uma política trivial [GLD00]. 24

5.1 Duas formas de particionamento do algoritmo de Iteração de Política Assíncrono. . . 32

5.2 BMDP da Figura 4.1 particionado de formas diferentes. (a) Particionamento aleatório; (b) Particionamento topológico. . . . . . . . . . . . . . . . . . . 35

6.1 Exemplo de um problema BMDP no domínio Layered com 2 camadas e 4 SCCs. . . 40

6.2 Exemplo de uma instância do domínio Grid World com 90 estados. . . . . . . . . . . 40

6.3 Exemplo de uma instância do domínio Race Track com 1239 estados. . . . . . . . . . . 41

6.4 Tempo de convergência para 4 instâncias do domínio Layered com 10000, 20000, 30000 e 40000 estados, variando o número de subconjuntos. Linhas verticais indicam o desvio padrão. . . . . . . . . . . . . . . . . . . . . . . . . . 42

6.5 Tempo de convergência para 4 instâncias do domínio Grid World com 9000, 18000, 27000 e 36000 estados, variando o número de subconjuntos. Linhas verticais indicam o desvio padrão. . . . . . . . . . . . . . . . . . . . . 43

6.6 Tempo de convergência para 4 instâncias do domínio Race Track com 1239, 5348, 11491 e 25832 estados, variando o número de subconjuntos. Linhas verticais indicam o desvio padrão. . . . . . . . . . . . . . . . . . . . . . . . 44 44

6.7 Tempo de convergência para 3 instâncias do domínio Tratamento Médico com 289, 1729 e 7201 estados, variando o número de subconjuntos. Linhas verticais indicam o

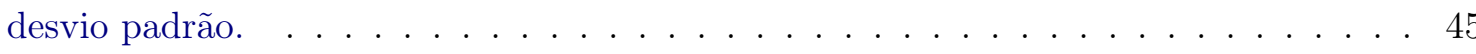


6.8 Tempo de convergência de RPI, RAPI* e RTPI em 4 domínios. (a) Domínio Layered variando o número de camadas; (b) Domínio Layered variando o número de estado e $n_{l}=10$; (c) Domínio Grid World variando o número de estados; (d) Domínio Race Track variando o número de estados; e (e) Domínio Médico variando o número de

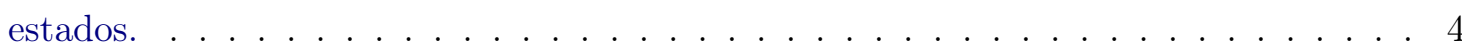




\section{Capítulo 1}

\section{Introdução}

$\mathrm{Na}$ área de planejamento probabilístico e aprendizado por reforço, os problemas geralmente são modelados como Processos de Decisão Markovianos (MDPs). Um MDP modela a interação de um agente com o ambiente. Em cada passo, uma ação é escolhida em um estado, produzindo uma recompensa e levando o sistema a um novo estado de acordo com os efeitos probabilísticos da ação escolhida. O propósito do agente é encontrar uma política que maximiza a recompensa acumulada esperada sobre um ambiente considerando um horizonte finito, indefinido ou infinito.

Algoritmos clássicos para resolver MDPs são os algoritmos de Iteração de Política (PI) e Iteração de Valor (VI) [Put94b]. O algoritmo PI aplica duas operações de maneira iterativa para todo o conjunto de estados: avaliação de política e melhoria de política, que correspondem a atualização da função valor e atualização da política, respectivamente. Embora PI garanta o retorno de uma política ótima, este processo pode levar a atualizações desnecessárias e algumas vezes apresentar problemas de escalabilidade, não conseguindo resolver de forma eficiente problemas com um número grande de estados. Uma possibilidade é utilizar algoritmos de PI assíncronos, que podem atualizar a política e a função valor de um subconjunto do espaço de estados de forma independente dos demais estados, a fim de reduzir o número total de operações. Nestes algoritmos, uma atualização da política e função valor para um subconjunto dos estados é chamada de meta-atualização. Um exemplo de PI assíncrono é o algoritmo proposto em [BY10b], que particiona o espaço de estados $S$ em subconjuntos $S_{i}$ e executa cada partição independentemente, além de permitir o uso de computação paralela.

Outra dificuldade encontrada para modelagem de MDPs refere-se a limitações na obtenção de probabilidades precisas para representação da transição de estados ou da falta de confiabilidade nas informações existentes. Nestes casos, pode ser necessário trabalhar com probabilidades imprecisas.

Exemplo 1. Sistema de navegação [DdBDS16] Em um sistema de navegação automático, as probabilidades de alcançar um determinado local após um movimento pode mudar dependendo de fatores do ambiente (tais como o tempo e condições da pista), e isso pode tornar a navegação mais difícil e sujeita a falhas. É difícil modelar todas estas mudanças precisamente uma vez que elas podem incluir muitas dependências externas. Em vista disso, poderia ser melhor ter uma política ótima robusta que considere conjuntos de probabilidades factíveis.

Entre as extensões de MDPs que consideram conjuntos de probabilidade estão: (i) os Processos de Decisão Markovianos com Probabilidades Imprecisas (Markov Decision Process with Imprecise transition Probabilities - MDP-IPs) [SL70] e (ii) os Processos de Decisão Markovianos com Probabilidades Intervalares (Bounded Parameter Markov Decision Process - BMDPs) [GLD00], que é um tipo especial de MDP-IP em que a função de probabilidades de transição é definida por um intervalo fechado de números reais. O critério de otimização geralmente utilizado em BMDPs é o critério maximin, que maximiza a recompensa considerando o pior cenário do modelo. Uma solução que utiliza tal critério é chamada de solução robusta. Este critério para BMDPs utiliza os limites do intervalo para avaliar a política de maneira gulosa, definindo as probabilidades precisas do modelo, ao invés de resolver uma otimização mais complexa necessária quando se resolve MDP-IPs em geral [GLD00, SL70, WED94]. 


\subsection{Motivação}

Givan et al. (2000) propuseram o algoritmo de Iteração de Valor Intervalar, um algoritmo síncrono para resolver BMDPs. Além deste algoritmo, existem algoritmos para a resolução de BMDPs de maneira mais eficiente, tais como LAO*[CSYL06], LRTDP Robusto [BA05], B²RTDP Robusto [FDdB14] e ILAO* Robusto [MDNdB16]. No entanto, estes algoritmos são destinados a problemas de horizonte indeterminado.

Bertsekas e Yu (2010) propuseram o algoritmo Asynchronous (Robust) Policy Iteration que pode ser utilizado para resolver uma variedade de problemas, entre eles: MDPs, MDPs direcionados a meta (Goal-Directed MDP - GMDP) e MDP-IPs. Este algoritmo assíncrono usa um sistema multiprocessador para executar meta-atualizações em cada subconjunto $S_{i}$ do conjunto de estados $S$. Para um MDP ou MDP-IP de horizonte infinito, Bertsekas e Yu (2010) provaram que o algoritmo converge sob as seguintes condições: (i) a atualização da política e função valor deve ser conservativa para garantir que a função valor de um estado $s \in S_{i}$ nunca decresça; (ii) a função valor deve ser monotônica; (iii) a função valor inicial deve estar entre um limite inferior e superior; (iv) o número de atualizações deve ser infinito para cada subconjunto $S_{i}$ do espaço de estados; e (v) a informação desatualizada sobre cada subconjunto $S_{i}$ eventualmente será limpada do sistema multiprocessador. Embora o trabalho de Bertsekas e Yu [BY10b] forneça resultados teóricos importantes, não há avaliação empírica dos algoritmos e não foram propostas formas de particionamento a serem utilizadas nos algoritmos.

\subsection{Objetivo}

O principal objetivo deste trabalho de mestrado é propor e avaliar algoritmos assíncronos eficientes em termos de tempo de execução baseados em iteração de política para resolução de BMDPs de horizonte infinito (usando a estratégia gulosa proposta por Givan et al. [GLD00]) com diferentes partições sobre o conjunto de estados.

\subsection{Principais contribuições}

As principais contribuições deste trabalho incluem:

- Proposição dos algoritmos Robust Asynchronous Policy Iteration (RAPI) e Robust Topological Policy Iteration (RTPI), versões assíncronas do algoritmo de Iteração de Política para BMDPs, baseados no particionamento do conjunto de estados.

- Análise do comportamento dos algoritmos propostos e do uso de programação paralela para resolução de BMDPs em domínios de planejamentos conhecidos e um novo domínio proposto.

\subsection{Organização}

A sequência deste trabalho está organizada da seguinte forma: nos capítulos 2, 3 e 4 são abordados respectivamente os modelos MDP, MDP-IP e BMDP, bem como algoritmos para resolvê-los. No capítulo 5 são descritos os algoritmos propostos, bem como a especificação dos operadores, heurísticas e provas de convergência. No capítulo 6 são apresentados os domínios de planejamento utilizados, assim como os experimentos realizados. Finalmente, no capítulos 7 são apresentados os trabalhos correlatos e no capítulo 8 as considerações finais são discutidas. 


\section{Capítulo 2}

\section{Processos de Decisão Markovianos}

Na área de Planejamento em IA, quando a transição de estados envolve efeitos probabilísticos, os problemas geralmente podem ser modelados utilizando um Processo de Decisão Markoviano (Markov Decision Process - MDP). Um MDP fornece um arcabouço matemático para representação e resolução de problemas de tomada decisão sequencial sob incerteza em ambientes completamente observáveis [Put94a].

Neste capítulo, são definidos MDPs em duas formulações principais, os MDPs enumerativos e os MDPs fatorados, bem como algoritmos para resolvê-los. Neste trabalho, o foco será dado aos MDPs em sua forma enumerativa.

\subsection{Definição de MDP}

Definição 1. Processo de Decisão Markoviano. Um MDP [Put94b] é definido formalmente como uma tupla $M=\langle S, A, P, R, \gamma\rangle$, em que:

- $S$ é um conjunto finito discreto de estados observáveis, chamado de espaço de estados;

- $A$ é um conjunto finito de ações que podem ser executadas pelo agente;

- $P\left(s^{\prime} \mid s, a\right)$ é a função de transição que retorna a probabilidade de alcançar o estado $s^{\prime}$ partindo do estado $s$ quando o agente aplica a ação $a$;

- $R(s, a)$ é a função de recompensa que associa um valor real para cada par estado-ação; e

- $\gamma$ é o fator de desconto, definido por $0 \leq \gamma<1$.

Um MDP pode ser representado por um grafo, em que os estados são representados pelos vértices e as transições ao aplicar as ações são representadas pelas arestas. A Figura 2.1 mostra um exemplo de um MDP, em que $S=\left\{s_{0}, \ldots, s_{15}\right\}, A=\left\{a_{1}, a_{2}\right\}$ e os valores das probabilidades $P\left(s^{\prime} \mid s, a\right)$ são mostrados nas arestas e a recompensa para todo par estado-ação é 1. As ações não mostradas em um estado indicam a não aplicabilidade delas nesse estado.

Um MDP descreve a evolução de um sistema representado pela interação de um agente decisor com o ambiente por sucessivos estágios ou passos de decisão. A cada estágio, o agente executa uma ação em um estado, produzindo uma recompensa imediata e um estado futuro definido estocasticamente. Este processo estocástico é considerado markoviano quando as funções de probabilidade de transição e recompensa dependem apenas do estado atual do sistema e da ação selecionada pelo agente nesse estado [Put94a, MK12a]. A interação do agente com o ambiente é denominada execução do $M D P$, e o resultado gerado pela execução é denominado histórico de execução.

Definição 2. Histórico de Execução. Um histórico de execução de um MDP de acordo com o estágio $t \in \mathbb{N}$ é uma sequência $\left(\left(s^{0}, a^{0}\right), \ldots,\left(s^{t-1}, a^{t-1}\right), s^{t}\right)$ de pares de estados visitados e ação escolhida no estágio $0 \leq t^{\prime} \leq t-1$, mais o estado visitado no estágio $t$. A cada par $\left(s^{t^{\prime}}, a^{t^{\prime}}\right)$ é produzida uma recompensa $r^{t^{\prime}}$. 


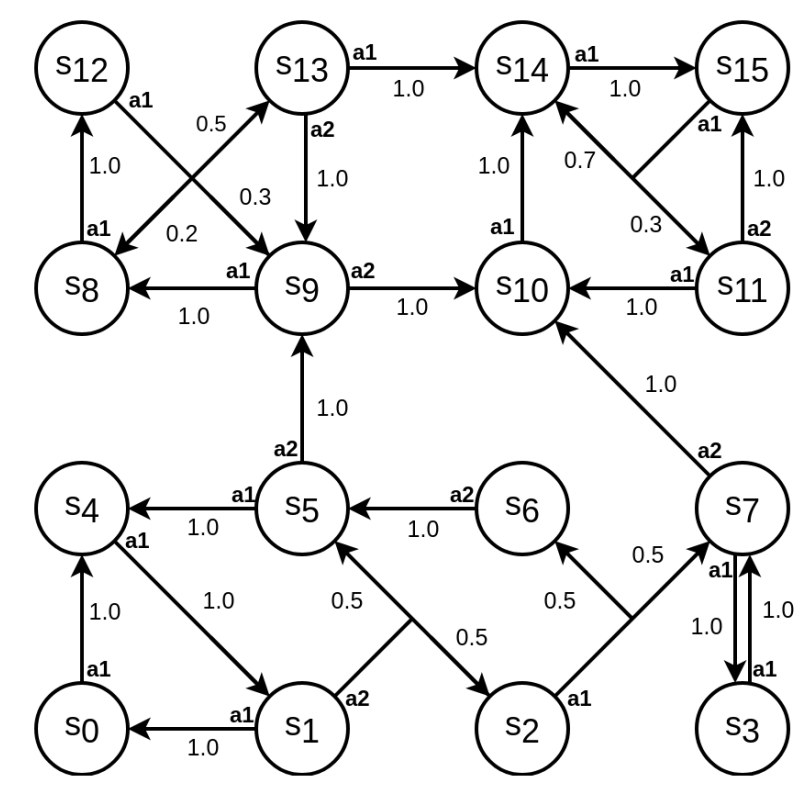

Figura 2.1: Exemplo do espaço de estados, ações e transiçôes de um MDP.

A cada execução de um MDP e o respectivo histórico de execução gerado, é produzida uma recompensa acumulada, determinada pela soma da recompensa de cada ação aplicada nos sucessivos estágios da execução. Um MDP pode ser classificado de acordo com o seu horizonte, o número de estágios pelos quais o agente tomará decisões. Um MDP de horizonte finito possui uma quantidade de passos de decisão limitado e conhecido. Um MDP de horizonte infinito possui um número irrestrito de passos de decisão, ou seja, não há um prazo para concluir o processo. Um MDP de horizonte indeterminado, comumente conhecido como SSP-MDP (Stochastic Shortest-Path MDP), possui a princípio um número infinito de passos de decisão, mas eventualmente é finalizado após alcançar um estado meta. Um SSP também pode ser interpretado como um problema com um número de passos de decisão finito mas desconhecido [MK12a].

O objetivo do agente é maximizar a recompensa acumulada esperada ao longo da sequência de ações escolhidas durante os históricos de execução. Para tal, ele deve determinar a melhor ação a ser executada em cada estado $s$ e estágio $t$. Uma regra que permite a seleção de uma ação em um estado e estágio é chamada de política. Assim, o objetivo do agente passa a ser encontrar uma política que maximize a recompensa acumulada esperada. A política pode ser não estacionária ou estacionária.

Definição 3. Política não estacionária. Uma política não estacionária é uma função $\pi: S \times \mathbb{N} \rightarrow$ $A$ que especifica uma ação $a=\pi(s, t)$ a ser escolhida em cada estado $s$ e estágio $t$.

Definição 4. Política estacionária. Uma política estacionária é uma função $\pi: S \rightarrow A$ que especifica uma ação $a=\pi(s)$ a ser escolhida em cada estado $s$ independentemente do estágio no qual se encontra.

A execução de uma política $\pi$ é a execução de um MDP em que para todo estágio $t$, as ações são especificadas por $a^{t}=\pi\left(s^{t}, t\right)$ para uma política não estacionária ou $a^{t}=\pi\left(s^{t}\right)$ para uma política estacionária. A medida de quão boa é uma política $\pi$ é definida pela utilidade proveniente de sua execução. Esta utilidade pode ser calculada como o valor esperado da recompensa acumulada sobre os possíveis históricos de execução a partir do estado $s$ e estágio $t$ ao executar a política $\pi$, denominado recompensa acumulada esperada.

Ao considerar um MDP de horizonte finito, o número de passos de decisão é limitado e portanto a recompensa acumulada esperada é finita, o que é passível de comparação. Por outro lado, em um MDP de horizonte infinito, o número de passos de decisão é infinito e a recompensa acumulada esperada pode ser infinita, o que torna necessário o uso do fator de desconto $0 \leq \gamma<1$ para que a 
recompensa acumulada convirja para um valor finito, o que é chamado de recompensa acumulada descontada esperada. Note que a recompensa acumulada em $t$ passos é descontada por $\gamma^{t}$. Sendo assim, o objetivo do agente pode ser interpretado como o ato de encontrar a política que maximiza a recompensa acumulada esperada ou recompensa descontada acumulada esperada, que também é conhecida como função valor.

Neste trabalho estamos interessados em MDPs de horizonte infinito. Isto significa que a partir deste ponto apenas políticas estacionárias serão consideradas como solução de um MDP, uma vez que, para avaliação da política, é considerado um número infinito de passos de decisão restantes, e por isso a ação escolhida independe do estágio.

Definição 5. Função valor de uma política. Dada uma política $\pi$, uma função valor $V^{\pi}: S \rightarrow \mathbb{R}$ associa uma utilidade ao executar a política $\pi$ a partir do estado $s$ indefinidamente, definida como recompensa descontada acumulada esperada e formalmente descrita por:

$$
V^{\pi}(s)=E_{\pi}\left[\sum_{t=0}^{\infty} \gamma^{t} r^{t} \mid s_{0}=s\right] .
$$

Seja $V(s)$ a função valor que represente uma estimativa qualquer da recompensa acumulada esperada de um estado no espaço de estados, e $V$ essa estimativa para todo o conjunto de estados. A função que calcula o valor de aplicar uma ação $a$ em um estado de $s$ é chamada de função qualidade.

Definição 6. Função qualidade. A qualidade da execução de uma ação $a$ num estado $s$ considerando uma função valor arbitrária $V$, provém da soma da recompensa imediata dessa execução com o valor esperado dos estados sucessores $s^{\prime}$, isto é:

$$
Q^{V}(s, a)=R(s, a)+\gamma \sum_{s^{\prime} \in S} P\left(s^{\prime} \mid s, a\right) V\left(s^{\prime}\right) .
$$

Com base na função qualidade, seja $T$ um operador sobre $V(s)$ definido por:

$$
(T V)(s)=\max _{a \in A}\left\{Q^{V}(s, a)\right\}
$$

e seja $\pi^{V}$ uma política gulosa com relação a uma função valor arbitrária $\mathrm{V}$ dada por:

$$
\pi^{V}(s)=\underset{a \in A}{\operatorname{argmax}}\left\{Q^{V}(s, a)\right\} .
$$

A solução ótima de um MDP é definida a seguir.

Definição 7. Função valor ótima e política ótima. A solução de um MDP de horizonte infinito é uma política ótima estacionária $\pi^{*}$ correspondente à função valor ótima $V^{*}(s)$, tal que $V^{*}$ domina as funções valor de todas as outras políticas, isto é, $V^{*}(s) \geq V^{\pi}(s)$ para todo estado $s$ e política $\pi$. $V^{*}(s)$ é o ponto fixo de $T$ (Equações 2.2 e 2.3), i.e., $V^{*}(s)$ satisfaz a seguinte equação, conhecida como o Princípio de Otimalidade de Bellman para MDPs ou Equação de Bellman [Put94b]:

$$
V^{*}(s)=\max _{a \in A}\left\{Q^{*}(s, a)\right\}=\max _{a \in A}\left\{R(s, a)+\gamma \sum_{s^{\prime} \in S} P\left(s^{\prime} \mid s, a\right) V^{*}\left(s^{\prime}\right)\right\},
$$

em que $Q^{*}(s, a)$ é a função qualidade ótima para o estado $s$ e ação $a$. Finalmente, uma política ótima pode ser considerada como uma política gulosa sobre a função valor ótima, e é calculada como:

$$
\pi^{*}(s)=\underset{a \in A}{\operatorname{argmax}}\left\{Q^{*}(s, a)\right\} .
$$




\subsection{Algoritmos para MDPs}

MDPs podem ser resolvidos por algoritmos baseados em programação dinâmica ou programação linear. Ambas as soluções são P-completas [PT87], ou seja, podem ser obtidas em tempo polinomial no pior caso.

Apesar de existirem vários tipos de soluções, MDPs geralmente são resolvidos por algoritmos de programação dinâmica, por serem passíveis de uma variedade maior de otimizações [MK12a]. Dentre eles há os algoritmos que utilizam atualizações síncronas, aqueles que atualizam todos os estados a cada iteração, e os que utilizam atualizações assíncronas, que podem atualizar os estados em diferentes ordens e realizar diferentes quantidades de atualizações.

Os algoritmos síncronos para resolução de MDPs são Iteração de Valor (VI) [Bel57] e Iteração de Política (PI) [How60]. Estes algoritmos servem de base para diversos outros algoritmos mais eficientes, que incluem Iteração de Política Assíncrono (API) [BY10a] e Iteração de Valor Topológico (TVI) [DG07], ambos assíncronos.

\subsubsection{Algoritmos síncronos baseados em programação dinâmica}

Os algoritmos síncronos de Iteração de Valor e Iteração de Política para resolução de MDPs possuem abordagens diferentes, porém complementares. Enquanto o algoritmo de Iteração de Valor pode ser entendido como um algoritmo que busca a função valor ótima no espaço de funções valor, o algoritmo de iteração de política pode ser entendido como um que busca a política ótima no espaço de políticas [MK12a].

\section{Iteração de Valor}

Dada uma função valor inicial, o algoritmo de Iteração de Valor (Value Iteration - VI) calcula iterativamente uma estimativa da função valor da iteração futura $t+1 \mathrm{com}$ base na função valor da iteração atual $t$, como segue:

$$
V^{t+1}(s)=\max _{a \in A}\left\{R(s, a)+\gamma \sum_{s^{\prime} \in S} P\left(s^{\prime} \mid s, a\right) V^{t}\left(s^{\prime}\right)\right\} .
$$

Este passo é executado até que a diferença máxima entre a função valor das iterações $t+1$ e $t$ para todos os estados seja menor que um fator de erro $\epsilon$ definido a priori, também conhecida como residual. Uma vez que a função valor tenha convergido, a política ótima é calculada por:

$$
\pi^{*}(s)=\underset{a \in A}{\operatorname{argmax}}\left\{R(s, a)+\gamma \sum_{s^{\prime} \in S} P\left(s^{\prime} \mid s, a\right) V^{t+1}\left(s^{\prime}\right)\right\} .
$$

Assim, o algoritmo de iteração de valor encontra uma política estacionária $\epsilon$-ótima dentro de um número finito de iterações, cada qual com complexidade $O\left(|S|^{2}|A|\right)$. Na prática, escolher um $\epsilon$ suficientemente pequeno garante que o algoritmo pare com uma política muito próxima da ótima [Put94a], embora isto faça com que o número total de iterações seja maior.

\section{Iteração de Política}

O algoritmo de Iteração de Política (Policy Iteration - PI) encontra uma política ótima aplicando iterativamente os seguintes passos a partir de uma política inicial arbitrária $\pi^{0}$ :

- Avaliação de política. Dada uma política $\pi^{t}$ em uma iteração $t$, o passo de avaliação de política calcula o valor de $\pi^{t}$, denotado por $V^{t}$. O passo de avaliação de política pode ser executado de maneira exata ou aproximada. O valor exato de $V^{t}$ pode ser obtido resolvendo um sistema de equações lineares, através de Eliminação Gaussiana ou outros algoritmos, que possuem em geral complexidade $O\left(|S|^{3}\right)$ [MK12b]. Por outro lado, o valor aproximado de $V^{t}$ pode ser calculado com um algoritmo iterativo baseado em programação dinâmica, que calcula uma aproximação em $\epsilon$ da função valor, similar ao algoritmo de iteração de valor. 
- Melhoria de política. O passo de melhoria de política é executado depois do cálculo da função valor $V^{t}$. Assim, este passo utiliza essa nova função valor para calcular uma nova política melhorada $\pi^{t+1}$. A complexidade computacional do passo de melhoria de política é $O\left(|S|^{2}|A|\right)$.

Enquanto for possível melhorar a política atual, o algoritmo irá encontrá-la e uma outra iteração será necessária. Caso contrário, se $\pi^{t+1}(s)=\pi^{t}(s)$ para todo $s \in S$, o algoritmo termina e a política encontrada é a ótima.

O algoritmo PI com avaliação de política iterativa é conhecido como Iteração de Política Modificado (Modified Policy Iteration - MPI). O algoritmo MPI, combina características dos algoritmos PI e VI. Assim como PI, o algoritmo contém um passo de melhoria de política e um passo de avaliação de política. Assim como VI, a função valor inicial é inicializada com um valor $V^{0}$ no passo de avaliação de política e possui o mesmo critério de parada, o que tem como resultado uma política $\epsilon$-ótima.

Após comparação da análise de convergência dos algoritmos MPI e VI, Puterman [Put94a] afirma que o algoritmo VI não deveria ser utilizado na prática, uma vez que a o algoritmo MPI é mais fácil de implementar e ainda possui desempenho superior. Daqui em diante o algoritmo MPI é denominado PI, uma vez que o que difere entre os dois algoritmos é apenas o passo de avaliação de política.

\subsubsection{Algoritmos assíncronos baseados em programação dinâmica}

Uma vez que os algoritmos síncronos atualizam todos os estados a cada iteração, eles eventualmente podem executar atualizações desnecessárias. Isso pode ocorrer quando um estado é atualizado antes de que os valores dos próximos estados tenham sido propagados, ou quando um estado continua a ser atualizado mesmo após a convergência [MK12b], i.e., um estado pode convergir antes de outros estados, mas o algoritmo síncrono irá parar apenas quando a política completa tiver convergido. Este problema acontece principalmente em MDPs cujo grafo de estados é acíclico ou a matriz de transição é esparsa [WS05].

Em geral é possível explorar as características inerentes de um MDP para produzir algoritmos eficientes para resolvê-los [LDK95]. A partir deste princípio, vários algoritmos assíncronos têm sido propostos. Alguns com foco na priorização da atualização de estados [WS05], outros pelo particionamento do conjunto de estados utilizando a estrutura do grafo do MDP [DG07], ou mesmo particionamentos arbitrários do conjunto de estados juntamente com a utilização de computação paralela [BY10a]. Neste trabalho, estamos interessados em algoritmos assíncronos baseados no particionamento do conjunto de estados, entre eles: Iteração de Política Assíncrona e Iteração de Valor Topológico.

\subsubsection{Iteração de Política Assíncrono Natural}

Um dos principais algoritmos de Iteração de Política Assíncrona é o de Williams e Baird (1993), conhecido como Iteração de Política Assíncrona Natural. Apesar de ser mostrado que o algoritmo falha em algumas circunstâncias, ele abriu novas possibilidades de pesquisa sobre o tema.

Em [WB93] são definidos três operadores. O operador de avaliação de política local para um único estado:

$$
\left(H^{\pi} V\right)(s)=Q^{V}(s, \pi(s)),
$$

o operador de melhoria de política local para um par (estado, ação):

$$
\left(I^{V} \pi\right)(s, a)= \begin{cases}a & \text { se } Q^{V}(s, a) \geq Q^{V}(s, \pi(s)) \\ \pi(s) & \text { caso contrário }\end{cases}
$$


e um operador de melhoria de política local para um único estado, considerando todas as ações:

$$
\left(I^{V} \pi\right)(s)=\max _{a \in A}\left(I^{V} \pi\right)(s, a) .
$$

O algoritmo aplica uma sequência infinita de operadores $H^{\pi}(s) \cup I^{V}(s, a)$ sob o par inicial $\left(\pi^{0}, V^{0}\right)$ e produz uma sequência de pares política-valor que convergiria para a otimalidade. Essa sequência de operadores consiste na aplicação alternada de: (i) qualquer sequência finita de operadores de $H^{\pi}(s)$; e (ii) qualquer sequência finita de operadores de $I^{V}(s, a)$.

O problema existente no algoritmo proposto por Williams e Baird (1993), é que sob certas condições iniciais maliciosas e dependendo da configuração do MDP, o algoritmo pode falhar, fazendo com que a política nunca convirja. O seguinte exemplo ilustra um desses problemas.

A Figura 2.2 representa o MDP em que é mostrado o contraexemplo de Williams e Baird (1993). Em cada estado $i$, a ação 1 causa uma transição determinística para o estado $(i-1) \bmod 6$ com recompensa 1 . Nos estados com numeração par, a ação 2 causa uma transição determinística para o estado $(i-2) \bmod 6$ cuja recompensa é 3. Do lado de cada nó é mostrado o valor inicial para aquele estado e que será usado no passo de avaliação da política. A política inicial é arbitrária.

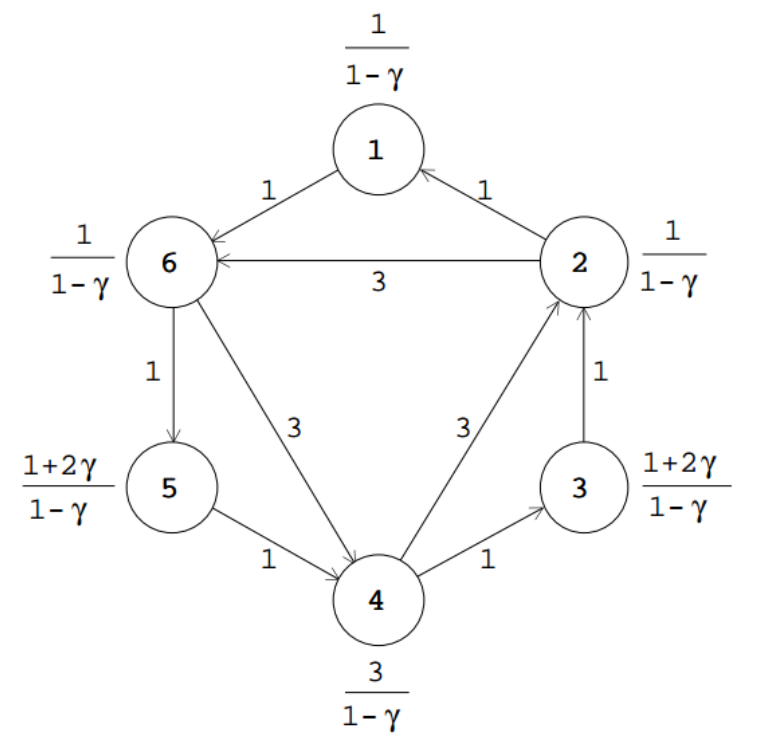

Figura 2.2: Contraexemplo de Williams e Baird [WB93].

A repetição da seguinte sequência de 18 operadores (em que $\pi$ foi omitido de $H^{\pi}$ e $V$ foi omitido de $I^{V}$ uma vez que $\pi$ e $V$ se referem a política e função valor atuais) faz com que a função valor retorne aos valores iniciais:

$$
\begin{aligned}
& H(2) H(5) I(5) H(1) I(1) H(1) H(2) I(2) H(4) I(4) \\
& H(6) H(3) I(3) H(5) I(5) H(5) H(6) I(6) H(2) I(2) \\
& H(4) H(1) I(1) H(3) I(3) H(3) H(4) I(4) H(6) I(6)
\end{aligned}
$$

De forma geral, qualquer que seja a sequência de operadores, as funções valores oscilarão entre $1 /(1-\gamma)$ e $(1+2 \gamma) /(1-\gamma)$ para estados com numeração ímpar e $1 /(1-\gamma)$ e $3 /(1-\gamma)$ para estados com numeração par, fazendo com que a convergência não seja sempre garantida.

\subsubsection{Iteração de Política Assíncrono}

A fim de superar o problema de convergência do algoritmo de Iteração de Política Assíncrono Natural, Bertsekas e Yu (2010) propõem o algoritmo Iteração de Política Assíncrono (Asynchronous 
Policy Iteration - API). API usa técnicas de computação multiprocessador para resolver um MDP de forma distribuída pelo particionamento do conjunto de estados $S$ em subconjuntos $S_{i}$, com $1 \leq i \leq n$. Então, a cada iteração, cada processador atualiza a política e a função valor de um único subconjunto $S_{i}$ até que a política parcial para este subconjunto convirja. O algoritmo API executa operações de atualização conservativas para garantir que a função valor de cada $s \in S_{i}$ nunca decresça [BY10b], garantindo a convergência da política ótima completa.

O algoritmo API é inicializado com uma política arbitrária $\pi^{0}$ e uma função valor inicial $V^{0}$ que está entre um limite inferior e superior da recompensa descontada acumulada esperada e executa meta-atualizações conservativas sobre um subconjunto $S_{i}$, como descrito a seguir.

Melhoria de politica conservativa. Para cada $s \in S_{i}$ a política $\pi^{t}$ é melhorada por:

$$
\pi^{t+1}(s)=\underset{a \in A}{\operatorname{argmax}}\left\{R(s, a)+\gamma \sum_{s^{\prime} \in S} P\left(s^{\prime} \mid s, a\right) V^{t}\left(s^{\prime}\right)\right\},
$$

e depois, a função valor é atualizada por:

$$
V^{t+1}(s)=\max _{a \in A}\left\{R(s, a)+\gamma \sum_{s^{\prime} \in S} P\left(s^{\prime} \mid s, a\right) V^{t}\left(s^{\prime}\right)\right\} .
$$

O valor $V^{t+1}(s)$ é então armazenado em uma função valor auxiliar $V_{a u x}^{t+1}(s)$, para ser utilizado no passo de avaliação de política conservativa.

Avaliação de política conservativa. Para garantir que o valor da política $\pi^{t+1}$ nunca decresça, este passo executa a seguinte atualização conservativa em todo estado $s \in S_{i}$ :

$$
V^{t+1}(s)=\max \left\{V_{a u x}^{t+1}(s), R\left(s, \pi^{t+1}(s)\right)+\gamma \sum_{s^{\prime} \in S} P\left(s^{\prime} \mid s, \pi^{t+1}(s)\right) V^{t+1}\left(s^{\prime}\right)\right\},
$$

que também garante a convergência da política completa, causando uma redução no número total de atualizações [BY10b].

Note que para ambos os passos, o valor do conjunto completo de estados $S$ deve estar disponível para cada processador $\left(V^{t}\left(s^{\prime}\right)\right.$ é usado pelas Equações 2.12 e 2.14). Bertsekas e Yu [BY10a] provaram que mesmo considerando um atraso de comunicação e depois de infinitas meta-atualizações, o algoritmo converge (Teorema 1). A prova do Teorema 1 usa a Proposição 1.

Proposição 1. Para cada MDP de horizonte infinito valem as seguinte propriedades [Ber77, BS78, Ber82, Ber07]: (i) a função $Q^{V}$ (Equação 2.2) é monótona, i.e., se $V(s) \leq V^{\prime}(s)$ para todo $s \in S$ então $Q^{V}(s, a) \leq Q^{V^{\prime}}(s, a)$ para todo $s \in S$ e $a \in A$; (ii) o operador $T$ (Equação 2.3) tem um único ponto fixo $V^{*}$; e (iii) há duas funções $\underline{V}$ e $\bar{V}$ tais que $\underline{V} \leq V \leq \bar{V}, \underline{V} \leq T \underline{V} \leq T \bar{V} \leq \bar{V}$ e $\lim _{k \rightarrow \infty}\left(\left(T^{k} \underline{V}\right)(s)\right)=\lim _{k \rightarrow \infty}\left(\left(T^{k} \bar{V}\right)(s)\right)=\bar{V}^{*}(s), \forall s \in S$, em que $T^{k}$ é a composição do operador $T$, i.e. $\left(T^{k} V\right)(s)=\left(T\left(T^{k-1} V\right)\right)(s)$.

Teorema 1. Convergência dos algoritmos de Iteração de Política Assíncronas [BY10b]. Dado um MDP de horizonte infinito e a Proposição 1, os algoritmos de Iteração de Política Assíncrona que utilizam a melhoria de política conservativa e avaliação de política conservativa convergem se: (i) a função valor inicial $V_{i}^{0}$ para todo $s \in S_{i}, 1 \leq i \leq n$ satisfaz $\underline{V} \leq V_{i}^{0}(s) \leq \bar{V}$; e (ii) o número de atualizações de política é infinito e a troca de informação eventualmente ocorrerá.

Note que a primeira condição do Teorema 1 é trivialmente satisfeita usando um número $\beta$ suficientemente grande (um limite superior da recompensa descontada acumulada esperada) tal que $\underline{V}=-\beta$ e $\bar{V}=\beta$, por exemplo, $\beta=R_{\max } /(1-\gamma)$, em que $R_{\max }=\max _{s, a}\{|R(s, a)|\}$ [BY10b]. A segunda condição é satisfeita com infinitas atualizações de política e a troca de informação é garantida pelo sistema multiprocessador. 
Na prática, o algoritmo API é finalizado depois de um número finito de passos. Isto é feito executando os dois passos, avaliação de política e melhoria de política apenas uma vez, mas para todo estado $s \in S$, depois de executar uma meta-atualização sobre todos os subconjuntos $S_{i}$.

\subsubsection{Iteração de Valor Topológico}

O algoritmo de Iteração de Valor Topológico (Topological Value Iteration - TVI) [DMWG11] é um algoritmo assíncrono que divide o espaço de estados do MDP em Componentes Fortemente Conexos (Strongly Connected Components - SCCs) e atualiza cada componente sequencialmente em ordem topológica reversa.

Um SCC representa o agrupamento de estados que são mutualmente dependentes. Dados dois estados $s_{1}$ e $s_{2}$, e uma ação $a$, tais que $P\left(s_{2} \mid s_{1}, a\right)>0$, é dito que o estados $s_{2}$ é sucessor de $s_{1}$. A Equação 2.5 implica que a função valor de $s_{1}$ depende da função valor de $s_{2}$, isto é, $s_{1}$ é causalmente dependente de $s_{2}$. Além do mais, a relação de dependência causal é transitiva. Os estados $s_{1}$ e $s_{2}$ são mutualmente dependentes se $s_{1}$ e $s_{2}$ dependem causalmente um do outro.

A partir do agrupamento de estados mutualmente dependentes do conjunto de estados $S$, podese construir um grafo acíclico dirigido (Directed Acyclic Graph - DAG) G, em que cada SCC é um meta-estado. Seja $X$ e $Y$ diferentes meta-estados, se há dois estados $s_{1} \in X$ e $s_{2} \in Y$, e uma ação $a$ tal que $P\left(s_{2} \mid s_{1}, a\right)>0$, então há uma aresta conectando os meta-estados $X$ e $Y$. Observe que $G$ é acíclico, e que pelos Teoremas 2 e 3 existe uma ordem de atualização ótima. Assim, cada meta-estado precisa de apenas uma atualização.

Teorema 2. Ordem de atualização ótima (caso acíclico) [Ber01]: Dado um MPD acíclico, existe uma ordem de atualização ótima que, quando seguida, a função valor ótima e a política ótima podem ser calculadas executando apenas uma atualização por estado $s_{i}$.

Teorema 3. Ordem de atualização ótima (caso cíclico): Dado um MPD cíclico, e seu DAG correspondente, em que cada nó é um SCC (meta-estado), existe uma ordem de atualização ótima que, quando seguida, a função valor ótima e a política ótima podem ser calculadas executando apenas uma meta-atualização por meta-estado.

O Teorema 3 pode ser provado trivialmente seguindo o Teorema 2.

\subsubsection{Programação linear}

Além da programação dinâmica, como explicado anteriormente, é possível resolver um MDP com programação linear, também em tempo polinomial [PT87]. Embora a solução de um MDP com programação linear seja exata, o esforço computacional necessário para sua resolução pode ser demasiadamente grande para MDPs com um conjunto de estados grande.

A Equação de Bellman pode ser formulada como programação linear com a seguinte formulação primal:

$$
\begin{aligned}
& \min _{V^{*}}: \sum_{s} V^{*}(s) \\
& \text { s.t. : } V^{*}(s) \geq R(s, a)+\gamma \sum_{s^{\prime} \in S} P\left(s^{\prime} \mid s, a\right) V^{*}\left(s^{\prime}\right), \forall s \in S, a \in A .
\end{aligned}
$$

A solução de ponto fixo da Equação de Bellman pode ser encontrada ao forçar que $V^{*}(s)$ para todo $s \in S$ seja maior ou igual do que a soma da recompensa com o valor esperado de tomar cada ação $a \in A$, o que garante que $V^{*}(s)$ seja maior ou igual a maximização da mesma equação considerando cada ação $a \in A$. A minimização sobre $V^{*}(s)$ garante que a igualdade seja verdadeira. 


\subsection{MDP Fatorado}

Uma limitação dos MDPs é proveniente de sua especificação. A especificação de um MDP conforme a Definição 1 enumera todos os estados do conjunto de estados. O MDP com tal formulação é conhecido como MDP enumerativo ou flat. Além de enumerar todo o conjunto de estados, as funções de recompensa, funções de transição de estados (para cada ação) e funções valor para um MDP enumerativo são especificadas de forma tabular, em que o seu tamanho é definido de acordo com o tamanho do espaço de estados.

Especificar um MDP de forma enumerativa possui dois problemas principais: (i) o tamanho do conjunto de estados pode ser muito grande e (ii) a descrição do conjunto de estados é pouco informativa, o que pode impedir ou dificultar uma possível otimização do algoritmo [MK12a]. Para evitar esses problemas, é possível utilizar uma representação fatorada do conjunto de estados a partir de variáveis de estados relevantes para o sistema. Um MDP com representação de estados fatorada é chamado de Processo de Decisão Markoviano Fatorado (MDP Fatorado) [BHD99]. Tanto a representação fatorada de um MDP como os seus algoritmos podem ser denominados simbólicos ou estruturados.

Num MDP Fatorado, o espaço de estados é descrito por um conjunto de variáveis de estado $X=\left\{X_{1}, X_{2}, \ldots, X_{n}\right\}$. Um estado é representado por um vetor $\vec{x}=\left(x_{1}, x_{2}, \ldots, x_{n}\right)$, em que $x_{i}$ é o valor da variável de estado $X_{i}$. Assim, todo estado $s \in S$ é expressado por $\vec{x}$. Embora neste trabalho seja assumido que as variáveis sejam binárias, é possível utilizar variáveis multivaloradas. A cardinalidade do espaço de estados $S$ é exponencial no número de variáveis de estado. Por exemplo, se $n$ é o número de variáveis e elas são binárias, existem $2^{n}$ valorações possíveis, isto é $2^{n}$ estados possíveis.

Além da descrição do espaço de estados de maneira fatorada, também é possível descrever as recompensas e transições de estado de maneira fatorada, se estas possuem dependência estrutural com relação ao espaço de estados. A função de recompensa fatorada $R(\vec{x}, a)$ pode ser definida pela soma de $\psi$ funções de recompensa locais, em que uma recompensa local $R_{j}(\vec{x}, a)$ é uma recompensa restrita a um subconjunto das variáveis de $X$. Assim, a função de recompensa é fatorada como:

$$
R(\vec{x}, a)=\sum_{j=1}^{\psi} R_{j}(\vec{x}, a) .
$$

A função de transição de estados de um MDP fatorado é representada por Redes Bayesianas Dinâmicas (Dynamic Bayesian Networks - DBNs). Para cada ação $a \in A$, é definido um DAG contendo duas camadas de vértices, uma representando as variáveis do estado atual $X_{i} \in X$ e outra representando as variáveis do próximo estado $X_{i}^{\prime} \in X^{\prime}$. As arestas definem as dependências entre os vértices, sendo permitidas arestas das variáveis da primeira camada para as da segunda, e da segunda para ela mesma desde que ciclos não sejam formados. Seja $p a_{a}\left(X_{i}^{\prime}\right)$ os pais de $X_{i}^{\prime}$ em relação a ação $a$, isto é, o conjunto de variáveis das quais a variável $X_{i}^{\prime}$ é dependente para a ação especificada. Dado que o grafo obedece a propriedade de Markov, a probabilidade de transição pode ser fatorada como:

$$
P\left(\vec{x}^{\prime} \mid \vec{x}, a\right)=\prod_{i=1}^{n} P\left(x_{i}^{\prime} \mid p a_{a}\left(X_{i}^{\prime}\right), a\right) .
$$

Ainda assim, representar as probabilidades de transição exige que sejam especificadas tabelas de probabilidade condicional (Conditional Probability Tables - CPTs) para cada DBN, o que pode ser muito custoso. Uma forma de deixar esta representação mais compacta é através do uso de Diagramas de Decisão Algébrica (Algebraic Decision Diagrams - ADDs) [BFG+93], que também podem ser utilizados para representar as recompensas e a função valor.

Um ADD é um DAG com dois tipos de nós: nós de decisão, que contém uma variável $X_{i}$; e nós folha, que contém um valor real. Um ADD $F$ com $n$ variáveis booleanas representa uma função $f:\{0,1\}^{n} \rightarrow \mathbb{R}$. Além de deixar a representação de funções de forma compacta, ADDs 
permitem realizar de maneira eficiente operações unárias como $\min (\cdot), \max (\cdot)$, restrição $\left(\left.F\right|_{X_{i}=x_{i}}\right)$ e marginalização $\left(\sum_{x_{i}} F\right)$; e operações binárias como adição $(\oplus)$, subtração $(\ominus)$, multiplicação $(\otimes)$, $\min (\cdot, \cdot)$ e $\max (\cdot, \cdot)$. Todas essas operações são fechadas para ADDs.

Assim como em MDPs, MDPs fatorados podem ser resolvidos com programação dinâmica ou programação linear. No entanto, nesta seção será mostrado apenas o algoritmo SPUDD que é baseado em programação dinâmica.

O algoritmo SPUDD (Stochatic Planning Using Decision Diagrams) [HSAHB99] é uma adaptação do algoritmo de Iteração de Valor para MDP Fatorado, em que as operações são realizadas com o uso de ADDs. Para tal, a Equação de Bellman, descrita pela Equação 2.7, é reescrita em termos de ADDs:

$$
V_{D D}^{t+1}(\vec{x})=\max _{a \in A}\left\{R_{D D}(\vec{x}, a) \oplus \gamma \sum_{\vec{x}^{\prime}} \bigotimes_{i=1}^{n} P_{D D}\left(x_{i}^{\prime} \mid p a_{a}\left(X_{i}^{\prime}\right), a\right) V_{D D}^{t}(\vec{x})^{\prime}\right\}
$$

em que as variáveis com índice DD indicam funções representadas por ADDs. Para calcular $V_{D D}^{t}$ são necessárias apenas as operações de multiplicação, soma, maximização e marginalização.

Assim como o algoritmo de Iteração de Valor, primeiro as funções valores são inicializadas com uma função valor $V^{0}$. O algoritmo é então executado até que o Erro de Bellman seja menor do que o $\epsilon$ especificado. A cada iteração, as operações simbólicas são aplicadas em bloco para todos os estados. Em questão de complexidade computacional, no pior caso o SPUDD mantém a mesma complexidade dos algoritmos de iteração de valor. 


\section{Capítulo 3}

\section{Processos de Decisão Markovianos com Probabilidades Imprecisas}

Conforme apresentado no capítulo 2, os MDPs fornecem tanto as garantias teóricas quanto meios práticos eficientes para a modelagem e resolução de problemas de tomada de decisão sequencial. No entanto, nem sempre é possível modelar problemas como um MDP.

MDPs apresentam limitações de modelagem quando a representação das crenças é incompleta, ambígua ou contraditória. Estas limitações podem ser referentes tanto a representação das preferências (recompensas) que podem não ser representadas por valores numéricos precisos, quanto a dinâmica do sistema (dada pelas probabilidades de transição) que pode não ser representada por probabilidades precisas. Neste trabalho as imprecisões serão abordadas somente em relação às probabilidades de transição.

Para modelar esse tipo de problema são utilizados os Processos de Decisão Markovianos com Probabilidades Imprecisas (Markov Decision Processes with Imprecise Probabilities - MDP-IPs) [SL70, WED94].

\subsection{MDP-IP: Definição}

Um MDP-IP é uma extensão de um MDP, em que todos os elementos são definidos de forma semelhante a um MDP, com exceção das probabilidades de transição, as quais são definidas imprecisamente. Ao invés de ser utilizada uma única medida de probabilidade para cada transição de estados, é utilizado um conjunto de medidas de probabilidades, chamado de conjunto credal de transição.

Definição 8. Conjunto Credal. Um conjunto credal é definido como um conjunto de medidas de probabilidades viáveis, que satisfazem um conjunto de equações e inequações lineares.

Por exemplo, seja $X$ uma variável aleatória, cujos valores são $x_{1}, x_{2}$ e $x_{3}$, e seja $P(X)$ a função densidade de probabilidade dada pelas probabilidades $P\left(x_{1}\right), P\left(x_{2}\right)$ e $P\left(x_{3}\right)$, sujeita ao conjunto de restrições lineares $\varphi=\left\{P\left(x_{1}\right) \leq 2 / 3, P\left(x_{3}\right) \leq 2 / 3,2 P\left(x_{1}\right) \geq P\left(x_{2}\right), P\left(x_{1}\right)+P\left(x_{2}\right)+P\left(x_{3}\right)=1\right\}$. O conjunto credal resultante $K(X)$ é representado pela região cinza da Figura 3.1, que compreende todas as triplas válidas $\left\{P\left(x_{1}\right), P\left(x_{2}\right), P\left(x_{3}\right)\right\}$.

Ao especializar o conceito de conjuntos credais no contexto de MDPs é possível definir um modelo de transição impreciso.

Definição 9. Conjunto Credal de Transição. Um conjunto credal de transição é um conjunto credal que define as distribuições condicionais sobre o próximo estado $s^{\prime}$, dado um estado $s$ e ação $a$ e é denotado por $K\left(s^{\prime} \mid s, a\right)$.

Neste trabalho, é assumido que conjuntos credais de transição $K\left(s^{\prime} \mid s, a\right)$ são estacionários.

Definição 10. Processo de Decisão Markoviano com Probabilidades Imprecisas. Um MDP-IP é definido formalmente como uma tupla $\mathcal{M}_{\mathcal{I P}}=\langle S, A, K, R, \gamma\rangle$, em que: 


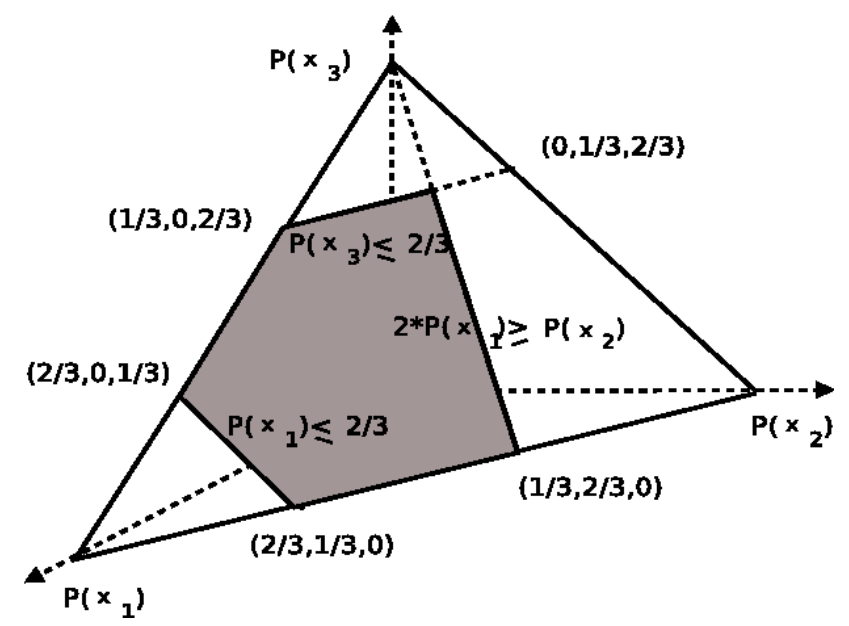

Figura 3.1: Exemplo de conjunto credal representado pela região cinza [DSdB11a].

- $S$ é um conjunto finito discreto de estados observáveis, chamado de espaço de estados;

- A é um conjunto finito de ações que podem ser executadas pelo agente;

- $K\left(s^{\prime} \mid s, a\right)$ é o conjunto credal de transição que define as distribuições condicionais sobre o próximo estado $s^{\prime}$, dado um estado $s$ e a ação $a$;

- $R(s, a)$ é a função de recompensa que associa um valor real para cada par estado-ação; e

- $\gamma$ é o fator de desconto, definido por $0 \leq \gamma<1$.

A Figura 3.2, mostra uma adaptação do MDP da Figura 2.1, em que probabilidades de transição são definidas por conjuntos credais de transição.

Um MDP-IP pode ser formulado baseado em Teoria dos Jogos ou de forma Bayesiana [SL70]. O restante do texto deste capítulo está focado na abordagem baseada em Teoria dos Jogos.

\subsubsection{Critérios}

Na formulação baseada em Teoria dos Jogos, o MDP-IP é formulado como um jogo entre duas entidades: o agente e a natureza. Entende-se por natureza, o mecanismo que escolhe a probabilidade de transição após a escolha da ação pelo agente.

Para que se possa avaliar uma política em um MDP-IP é preciso fazer suposições sobre o comportamento da natureza, o que pode gerar diferentes critérios de otimização. Dentre os possíveis comportamentos da natureza, nos extremos pode-se pensar em uma natureza totalmente cooperativa e uma natureza totalmente competitiva. Uma natureza totalmente cooperativa escolhe as probabilidades que maximizarão a recompensa acumulada esperada do tomador de decisão, enquanto que a natureza totalmente competitiva escolhe as probabilidades que minimizarão sua recompensa acumulada esperada. É possível pensar também em um comportamento híbrido da natureza, a partir da combinação dos comportamentos extremos. Assim, dentre os possíveis critérios de otimização, destacam-se:

- Critério maximin [SL70]: considera o comportamento competitivo da natureza, i.e., o agente escolhe a ação que maximiza a recompensa acumulada esperada com base na distribuição de probabilidades menos favorável. Neste caso, o agente é considerado pessimista, uma vez que considera o pior cenário possível para otimização. Este critério também é chamado de critério robusto.

- Critério maximax [SL70]: considera o comportamento cooperativo da natureza, i.e., o agente escolhe a ação que maximiza a recompensa acumulada esperada com base na distribuição 


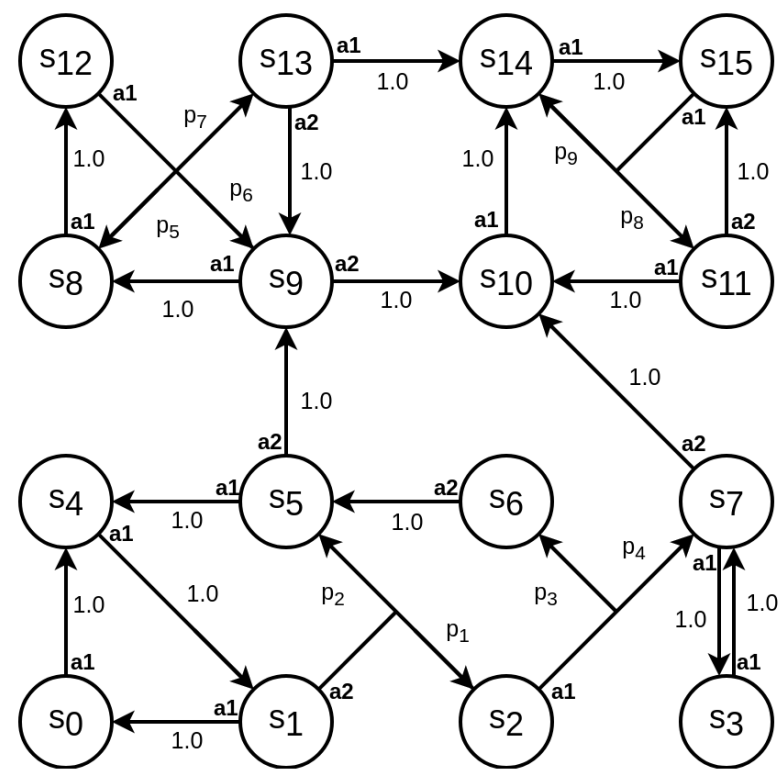

(a)

\author{
Restrições $\varphi_{1}$ \\ $\mathrm{p}_{1}<=0.6$ \\ $p_{2}>=0.4$ \\ $p_{1}+p_{2}=1$
}

\section{Restrições $\varphi_{2}$}

$p_{3}<=0.5$

$\mathrm{p}_{3}+\mathrm{p}_{4}=1$

Restrições $\varphi_{3}$

$p_{5}<=0.5$

$\mathrm{p}_{7}<=0.6$

$2 * p_{5}>=p_{6}$

$p_{5}+p_{6}+p_{7}=1$

Restrições $\varphi_{4}$

$p_{8}+p_{9}=1$

(b)

Figura 3.2: Exemplo de MDP-IP. a) Espaço de estados, ações e transições de um MDP-IP. b) Conjunto de restrições lineares $\varphi_{i}$ referentes aos conjuntos credais de transição.

de probabilidades mais favorável. Neste caso, o agente é considerado otimista, uma vez que considera o melhor cenário possível para otimização.

- Critério maximix [KCdC05]: considera um comportamento híbrido da natureza, i.e., o agente considera a política que produz o máximo de $\alpha$ vezes o critério maximax mais $(1-\alpha)$ vezes o critério maximin para algum fator $\alpha \in(0,1)$.

\subsubsection{Critério Robusto}

O método maximin é importante na área de inteligência artificial, como um método que considera a maximização do ganho mínimo. No contexto de MDP-IPs, o critério maximin representa um resultado robusto sob a incerteza dada por $K$, em que o objetivo do agente é maximizar a recompensa esperada ao considerar a situação mais adversa possível. Neste trabalho o critério de otimização pessimista será utilizado como padrão para resolução de MDP-IPs.

Em um MDP a qualidade $Q$ de executar uma ação $a$ em um estado $s$ é calculada como a soma de sua recompensa imediata com o valor esperado dos estados sucessores (Equação 2.2). Em um MDP-IP, a função qualidade de um par $(s, a)$ considera a escolha competitiva da natureza, e é denotada por função qualidade robusta.

Definição 11. Função qualidade robusta. Dada uma função valor $V$, a qualidade robusta de executar uma ação $a$ num estado $s$ provém da soma da recompensa imediata dessa execução com o valor esperado dos estados sucessores $s^{\prime}$, considerando uma minimização sobre as probabilidades $P \in K$, isto é:

$$
Q_{\text {robust }}^{V}(s, a)=\min _{P \in K}\left\{R(s, a)+\sum_{s^{\prime} \in S} P\left(s^{\prime} \mid s, a\right) V\left(s^{\prime}\right)\right\} .
$$

Com base na função qualidade robusta, seja $D$ um operador sobre $V(s)$, definido por:

$$
(D V)(s)=\max _{a \in A}\left\{Q_{\text {robust }}^{V}(s, a)\right\}
$$


Definição 12. Função valor robusta ótima e política robusta ótima. Uma solução robusta ótima de um MDP-IP, é uma política robusta ótima $\pi^{*}$ correspondente a uma função valor robusta ótima $V^{*}$ que domina as funções valor de todas as outras políticas robustas, isto é, $V^{*}(s) \geq V^{\pi}(s)$ para todo estado $s$ e política $\pi$ considerando o critério maximin. $V^{*}(s)$ corresponde ao ponto fixo do operador $D$ (Equações 3.1 e 3.2) e satisfaz o princípio de otimalidade de Bellman para MDP-IPs [SL70]:

$$
V^{*}(s)=\max _{a \in A}\left\{Q_{\text {robust }}^{V^{*}}(s, a)\right\}=\max _{a \in A} \min _{P \in K}\left\{R(s, a)+\sum_{s^{\prime} \in S} P\left(s^{\prime} \mid s, a\right) V^{*}\left(s^{\prime}\right)\right\} .
$$

Uma política ótima é calculada como uma política gulosa sobre a função valor robusta ótima:

$$
\pi^{*}(s)=\underset{a \in A}{\operatorname{argmax}}\left\{Q_{\text {robust }}^{V^{*}}(s, a)\right\} .
$$

\subsection{Algoritmos}

Analogamente a MDPs, existem algoritmos baseados em programação dinâmica [SL70, WED94] e programação matemática [SCT $\left.{ }^{+} 07\right]$ para resolver MDP-IPs.

\subsubsection{Iteração de Política para MDP-IP}

O algoritmo de Iteração de Política para MDP-IPs [SL70], que considera o critério maximin será referido como Iteração de Política Robusto (Robust Policy Iteration - RPI). RPI é uma extensão do algoritmo de Iteração de Política para MDPs [How60].

O algoritmo RPI possui os passos de avaliação de política robusta e melhoria de política robusta. Dada uma política inicial $\pi^{0}$, o algoritmo iterativamente melhora a política executando os dois passos a seguir até que a política resultante de duas iterações seguidas sejam idênticas:

Avaliação de política robusta. Dada uma política $\pi^{t}$ em uma iteração $t$, o passo de avaliação de política robusta encontra a função valor $V^{t}$ considerando um agente competitivo:

$$
V^{t}(s)=Q_{\text {robust }}^{V^{t}}\left(s, \pi^{t}(s)\right)
$$

Melhoria de política robusta. O passo de melhoria de política robusta calcula uma política melhorada $\pi^{t+1}$ com base na função valor $V^{t}$ calculada no passo de avaliação de política robusta:

$$
\pi^{t+1}(s)=\underset{a \in A}{\operatorname{argmax}}\left\{Q_{\text {robust }}^{V^{t}}(s, a)\right\} .
$$

A complexidade computacional do passo de avaliação de política é polinomial no número de es$\operatorname{tados}|S|$ e no número de probabilidades viáveis $|K|$, i.e. probabilidades que cumprem as restrições. O passo de melhoria de política, por sua vez, é polinomial no número de estados $|S|$ e ações $|A|$. Sendo assim, a complexidade computacional de cada iteração é polinomial e, desde que o número de iterações seja polinomial, o algoritmo é polinomial [Sch15]. No entanto, encontrar as probabilidades para calcular $Q_{\text {robust }}^{V^{t}}\left(s, \pi^{t}(s)\right)$ (Equação 3.5) é custoso computacionalmente pois implica em chamar um otimizador matemático. Assim em geral esse algoritmo é limitado a problemas pequenos $\left[\mathrm{SCT}^{+} 07\right]$.

\subsubsection{Iteração de Valor para MDP-IP}

Dada uma função valor inicial $V^{0}$, o algoritmo de Iteração de Valor para MDP-IPs calcula uma estimativa da função valor na iteração $t+1$ com base no valor na iteração atual $t$ :

$$
V^{t+1}(s)=\max _{a \in A} \min _{P \in K}\left\{R(s, a)+\gamma \sum_{s^{\prime} \in S} P\left(s^{\prime} \mid s, a\right) V^{t}\left(s^{\prime}\right)\right\} .
$$


Em cada iteração do algoritmo, a função valor futura $V^{t+1}$ é atualizada com o valor obtido pela ação que maximiza o valor correspondente ao pior caso da distribuição de probabilidade com relação à função valor da iteração anterior $V^{t}$. O restante do algoritmo funciona de forma equivalente à sua versão para MDP.

\subsubsection{Programação matemática}

Diferentemente de MDPs, resolver um MDP-IP implica em resolver um problema não linear, uma vez que além da otimização sobre a escolha de ações $a \in A$, é necessário fazer uma otimização sobre o conjunto de probabilidades $P \in K$. Existem duas duas formulações para resolver MDPIPs, uma baseada em programação de duas camadas e outra baseada em programação multilinear $\left[\mathrm{SCT}^{+} 07\right]$.

A Equação de Bellman para MDP-IPs (Equação 3.3) pode ser formulada com um problema de programação de dois níveis ao considerar $P$ como uma variável e adicionar um nível de otimização que minimiza sobre as probabilidades:

$$
\begin{aligned}
\min _{V^{*}}: & \sum_{s} V^{*}(s) \\
\text { s.t. }: & V^{*}(s) \geq R(s, a)+\gamma \sum_{s^{\prime} \in S} P\left(s^{\prime} \mid s, a\right) V^{*}\left(s^{\prime}\right), \forall s \in S, a \in A \\
& P\left(s^{\prime} \mid s, a\right)=\underset{W}{\operatorname{argmin}} \sum_{s^{\prime} \in S} W\left(s^{\prime} \mid s, a\right) V^{*}\left(s^{\prime}\right) \\
& \text { s.t. }: W\left(s^{\prime} \mid s, a\right) \in K\left(s^{\prime} \mid s, a\right) .
\end{aligned}
$$

Equivalentemente, é possível reduzir o problema de dois níveis para um problema de programação multilinear $\left[\mathrm{SCT}^{+} 07\right]$ :

$$
\begin{aligned}
\min _{V^{*}, P}: & \sum_{s} V^{*}(s) \\
\text { s.t. }: & V^{*}(s) \geq R(s, a)+\gamma \sum_{s^{\prime} \in S} P\left(s^{\prime} \mid s, a\right) V^{*}\left(s^{\prime}\right), \forall s \in S, a \in A \\
& P\left(s^{\prime} \mid s, a\right) \in K\left(s^{\prime} \mid s, a\right)
\end{aligned}
$$

Apesar de obter resultados exatos, essas soluções exigem muito esforço computacional, e portanto apenas problemas pequenos podem ser resolvidos de forma viável [SCT $\left.{ }^{+} 07\right]$.

\subsection{MDP-IP Fatorado}

A representação mais natural dos estados de um MDP se dá pela representação fatorada em variáveis de estado, que considera as características necessárias do ambiente e suas possíveis valorações. A utilização deste tipo de representação em MDP-IPs dá origem aos MDP-IPs fatorados [DSdB11a].

As representações fatoradas dos estados, recompensas e funções valor de um MDP-IP fatorado são idênticas às de um MDP fatorado. Entretanto, enquanto em um MDP fatorado as probabilidades de transição são representadas por DBNs e mais eficientemente por ADDs, em um MDP-IP fatorado o conjunto credal de transição é representado por Redes Credais Dinâmicas (DCN - Dynamic Credal Networks) [Coz00, Coz05] e mais eficientemente por PADDs (Parametrized ADDs) [DSdB11a].

Analogamente a uma DBN, para cada ação $a \in A$ é definido um DAG de duas camadas, em que a primeira camada representa as variáveis do estado atual $X_{i}$, a segunda camada representa as variáveis do estado futuro $X_{i}^{\prime}$ e as arestas representam as dependências dos nós futuros em relação aos nós pais $p a_{a}\left(X_{i}^{\prime}\right)$. A diferença é que para cada variável futura $X_{i}^{\prime}$ em uma DCN, é definida uma CPT com probabilidades imprecisas, dada por um conjunto de parâmetros e um conjunto de 
restrições lineares sobre esses parâmetros, o que define um conjunto credal de transição fatorado para cada variável.

Definição 13. Conjunto Credal de Transição Fatorado. Um conjunto credal de transição fatorado define as distribuições condicionais para uma próxima variável $X_{i}^{\prime}$, dados seus pais $p a_{a}\left(X_{i}^{\prime}\right)$ para uma ação $a$ e é denotado por $K_{a}\left(x_{i}^{\prime} \mid p a_{a}\left(X_{i}^{\prime}\right), a\right)$.

A fim de representar compactamente uma DCN, são utilizados os PADDs, uma extensão de ADDs em que os nós folhas são representados por equações parametrizadas. Um PADD com $n$ variáveis booleanas representa uma função $f:\{0,1\}^{n} \rightarrow \mathbb{E}$, onde $\mathbb{E}$ é o espaço de equações parametrizadas. PADDs são fechados sob as operações binárias de adição $(\oplus)$, subtração $(\ominus)$ e produto $(\otimes)$ [DSdB11a], e sob as operações unárias de restrição $\left(\left.F\right|_{X_{i}=x_{i}}\right)$ e marginalização $\left(\sum(\Delta)\right)$. PADDs não são fechados sob as operações de minimização e maximização, tanto unárias como binárias. No entanto, para resolver MDP-IPs fatorados, não são necessárias essas últimas operações.

Vários algoritmos foram propostos para resolver MDP-IPs fatorados, entre eles os algoritmos SPUDD-IP [DSdBC09], OBJECTIVE-IP [DdBCS09, DSdB11a] e FactoredMPA [DdBCS09, DdBCS11a]. Apenas o algoritmo SPUDD-IP será apresentado nesta seção.

O algoritmo SPUDD-IP é uma versão do algoritmo SPUDD para MDP-IPs. Para isto, é preciso expressar a Equação 3.7 de forma fatorada:

$$
V_{D D}^{t}(\vec{x})=\max _{a \in A}\left\{R_{D D}(\vec{x}, a) \oplus \gamma \min _{P \in K}\left\{\sum_{\vec{x}^{\prime}} \bigotimes_{i=1}^{n} P_{D D}\left(x_{i}^{\prime} \mid p a_{a}\left(X_{i}^{\prime}\right), a\right) V_{D D}^{t-1}(\vec{x})^{\prime}\right\}\right\} .
$$

Tanto a recompensa como a função valor podem ser representadas por ADDs, chamadas de $R_{D D}$ e $V_{D D}$, respectivamente. A única estrutura que precisa necessariamente ser representada por PADDs é a função de transição, representada por $P_{D D}$. Note que $\sum_{\vec{x}^{\prime}} \bigotimes_{i=1}^{n} P_{D D}\left(x_{i}^{\prime} \mid p a_{a}\left(X_{i}^{\prime}\right), a\right) V_{D D}^{t-1}(\vec{x})^{\prime}$ é um PADD e após aplicar $\min _{P \in K}$ obtemos um ADD pois esta operação aplica a minimização das probabilidades em cada folha do PADD. Sendo assim, é possível representar $V_{D D}$ como um ADD.

Assim como o algoritmo de iteração de valor, primeiro as funções valores são inicializadas com uma função valor $V^{0}$. O algoritmo é então executado até que o Erro de Bellman seja menor do que o $\epsilon$ especificado. A cada iteração, as operações simbólicas são aplicadas para todos os estados, aproveitando da dependência local o que permite que os cálculos sejam realizados de maneira eficiente. O restante do algoritmo funciona de maneira análoga ao algoritmo SPUDD.

\subsection{Outros modelos - Subclasses de MDP-IP}

Os Bounded-parameter Markov Decision Processes (BMDP) [GLD00] e os Markov Decision Processes with Set-valued Transitions (MDPST) são subclasses importantes de MDP-IPs [TCdB07a]. Estes modelos podem ser resolvidos mais eficientemente com algoritmos específicos do que com os algoritmos para MDP-IP. Por outro lado, uma vez que em MDP-IPs são permitidas restrições lineares mais gerais do que em BMDPs e MDPSTs, os algoritmos que resolvem BMDPs e MDPSTs não podem ser utilizados para resolver MDP-IPs.

Em BMDPs as probabilidades de transição são definidas por intervalos, isto é, são limitadas por um limite inferior e um limite superior. Um exemplo de transição de um BMDP é $P\left(s_{1} \mid s_{0}, a\right) \in$ $[0.5,0.7]$. Um BMDP pode ser transformado em um MDP-IP trivialmente ao considerar as probabilidades definidas por intervalos como as restrições do MDP-IP.

Os MDPSTs possuem as probabilidades de transições definidas sob conjuntos de estados, por exemplo, $P\left(\left\{s_{1}, s_{2}\right\} \mid s_{0}, a\right)=0.6$. A transição para qual estado dentro do conjunto de estados é escolhida de forma não-determinística. Um MDPST também pode ser transformado em um MDPIP ao considerar restrições sobre o conjunto de probabilidades de cada estado do conjunto de estados.

A Figura 3.3 (adaptada de [TCdB07a]) mostra o relacionamento entre os modelos usados em planejamento probabilístico em Inteligência Artificial. Os modelos BMDP e MDPST englobam a 


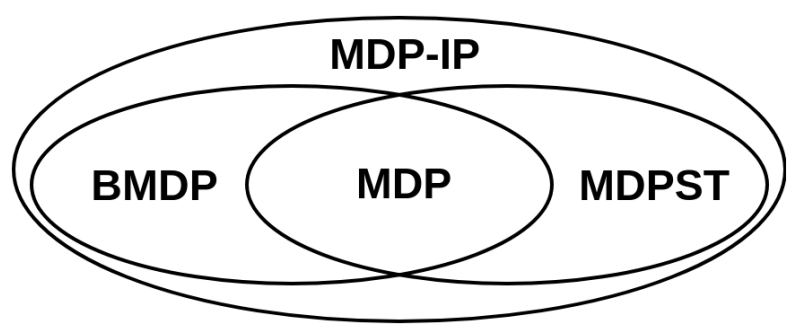

Figura 3.3: Relações entre diferentes modelos usados em planejamento probabilístico.

classe MDP e são subclasses estritas de MDP-IP. No entanto, BMDP e MDPST não são equivalentes, uma vez que possuem poder representativo diferente [TCdB07a].

É importante notar que um BMDP não pode ser representado de forma fatorada, uma vez que ao ter probabilidades de transição para cada variável de estado e cada ação de forma intervalar e calcular a probabilidade conjunta, não será obtida uma probabilidade de transição de estados definidas estritamente por intervalos, o que tornaria este modelo um modelo mais geral e complexo do que BMDPs.

No Capítulo 4 os BMDPs são apresentados com mais detalhes, uma vez que as contribuições deste trabalho são referentes a este modelo. 


\section{Capítulo 4}

\section{Processos de Decisão Markovianos com Probabilidades Intervalares}

Embora um MDP-IP possa representar uma gama maior de problemas em relação a um MDP, em geral eles são intratáveis quando possuem um espaço de estados muito grande, mesmo com as soluções mais eficientes para MDP-IP Fatorado.

Um caso especial de MDP-IP é o Processo de Decisão Markoviano com Probabilidades Intervalares (Bounded-Parameter Markov Decision Process - BMDP), que representa as probabilidades de transição de estados e recompensas por intervalos reais fechados. BMDPs podem ser resolvidos de forma menos custosa do que MDP-IPs e podem ser utilizados para (i) representação de imprecisão quando as crenças são incompletas, ambíguas ou contraditórias, assim como em MDP-IPs; e (ii) para representação de MDPs de forma compacta através da agregação de estados.

\subsection{BMDP: Definição}

Um BMDP é uma extensão de um MDP, em que as transições de estado e as recompensas são definidas por intervalos, e os demais elementos são definidos da mesma forma que em MDPs. Cada valoração dos intervalos de um BMDP define um MDP exato. Logo, um BMDP define um conjunto incontável de MDPs exatos.

Definição 14. Processo de Decisão Markoviano com Probabilidades Definidas por Intervalos. Um BMDP é definido formalmente como uma tupla $B=\left\langle S, A, P_{\uparrow}, R_{\uparrow}, \gamma\right\rangle$ [GLD00], em que:

- $S$ é um conjunto finito discreto de estados observáveis, chamado de espaço de estados;

- $A$ é um conjunto finito de ações que podem ser executadas pelo agente;

- $P_{\uparrow}\left(s^{\prime} \mid s, a\right)=\left[P_{\downarrow}\left(s^{\prime} \mid s, a\right), P_{\uparrow}\left(s^{\prime} \mid s, a\right)\right]$ é a função de probabilidade de transição intervalar do estado $s$ para o estado $s^{\prime}$ tomando a ação $a$. Os intervalos são fechados e $0 \leq P_{\downarrow}\left(s^{\prime} \mid s, a\right) \leq$ $P_{\uparrow}\left(s^{\prime} \mid s, a\right) \leq 1$

- $R_{\uparrow}(s, a)=\left[R_{\downarrow}(s, a), R_{\uparrow}(s, a)\right]$ é a função de recompensa que associa um intervalo real para cada par estado-ação; e

- $\gamma$ é o fator de desconto, definido por $0 \leq \gamma<1$.

Para garantir que a função de transição de estados seja bem formada, para todo par estado-ação deve ser garantido que [GLD00]:

$$
\begin{aligned}
& \sum_{s^{\prime} \in S} P_{\downarrow}\left(s^{\prime} \mid s, a\right) \leq 1 \\
& \sum_{s^{\prime} \in S} P_{\uparrow}\left(s^{\prime} \mid s, a\right) \geq 1
\end{aligned}
$$


Neste trabalho, é considerado que os valores dos limites inferior e superior dos intervalos das recompensas são os mesmos, portanto $R_{\uparrow}$ é equivalente a $R$ para MDPs. Assim, a única fonte de imprecisão na especificação do BMDP abordado daqui em diante é referente às transições de estado. Além disso, neste trabalho são considerados BMDPs de horizonte infinito.

A Figura 4.1 mostra um exemplo de um BMDP com 16 estados e probabilidades de transição definidas por intervalos.

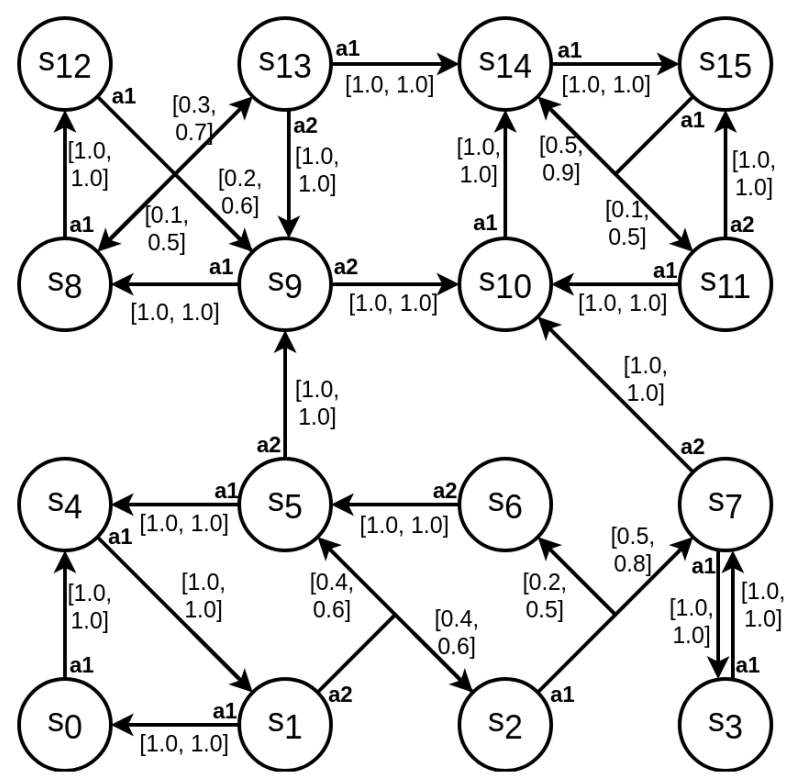

Figura 4.1: Exemplo do espaço de estados de um BMDP. As probabilidade de transição são rotuladas com intervalos, por exemplo, [0.2, 0.5] (o intervalo [1.0, 1.0] indica uma transição determinística).

O conjunto de MDPs definido por um BMDP é denotado por $M_{\uparrow}$. Para um MDP exato $M \in M_{\uparrow}$, as probabilidades de transição são denotadas por $P^{M}$. Dada uma política $\pi$, para cada MDP $M \in M_{\uparrow}$ é possível obter uma função valor $V^{\pi, M}$, isto é, a função valor da política $\pi$ para o MDP exato $M$. Considerando todos os MDPs possíveis, a função valor mínima e a função valor máxima obtidas para cada estado $s$ definem o limite inferior $V_{\downarrow}^{\pi}(s)$ e o limite superior $V_{\uparrow}^{\pi}(s)$ da função valor intervalar $V_{\uparrow}^{\pi}(s)=\left[V_{\downarrow}^{\pi}(s), V_{\uparrow}^{\pi}(s)\right]$. Apesar do número de MDPs possíveis em $M_{\uparrow}$ ser em geral incontável, apenas um subconjunto finito de $M_{\uparrow}$ é relevante para o cálculo de $V_{\uparrow}$ [GLD00].

Dada uma ordenação $O$ dos estados, um MDP de maximização de ordem é um MDP que tenta atribui maior massa de probabilidade para os primeiros estados da ordenação e menor massa de probabilidade para os últimos.

Definição 15. MDP de maximização de ordem. Formalmente, um MDP de maximização de ordem é um MDP $M_{O} \in M_{\uparrow}$ que maximiza

$$
\sum_{i=1}^{m-1} P_{\uparrow}\left(s_{i}^{\prime} \mid s, a\right)+\sum_{i=m}^{k} P_{\downarrow}\left(s_{i}^{\prime} \mid s, a\right) \leq 1
$$

para a ordenação $O=s_{1}, s_{2}, \ldots, s_{k}$. A atribuição de probabilidades do estado $s$ e ação $a$ para o MDP $M_{O}$ é definida para todo $s_{j}^{\prime}$ como segue:

$$
P^{M_{O}}\left(s_{j}^{\prime} \mid s, a\right)= \begin{cases}P_{\uparrow}\left(s_{j}^{\prime} \mid s, a\right) & \text { se } j<m \\ 1-\left(\sum_{i=1}^{m-1} P_{\uparrow}\left(s_{i}^{\prime} \mid s, a\right)+\sum_{i=m+1}^{k} P_{\downarrow}\left(s_{i}^{\prime} \mid s, a\right)\right) & \text { se } j=m \\ P_{\downarrow}\left(s_{j}^{\prime} \mid s, a\right) & \text { se } j>m\end{cases}
$$

Uma vez que o espaço de estados é finito, o número de ordenações possíveis é finita e, portanto, 
o número de MDPs de maximização de ordem é finito. O conjunto de MDPs de maximização de ordem é denotado por $X_{M_{\uparrow}}$.

Dado o BMDP da Figura 4.1 e a ordenação $O=s_{13}, s_{9}, s_{8}, s_{1}, s_{2}, s_{3}, s_{4}, s_{5}, s_{6}, s_{7}, s_{10}, s_{11}, s_{12}, s_{14}, s_{15}$, a Figura 4.2a mostra a parte do BMDP que inclui o estado $s_{12}$ e ação $a_{1}$. A Figura $4.2 \mathrm{~b}$ mostra a parte do MDP de maximização de ordem correspondente. Note que os primeiros estados recebem maior massa de probabilidade enquanto os últimos recebem menor massa de probabilidade, segundo a restrição da Equação 4.2. No exemplo, o estado $s_{13}$ recebe $P_{\uparrow}\left(s_{13} \mid s_{12}, a_{1}\right)=0.7$, o estado $s_{8}$ recebe $P_{\downarrow}\left(s_{8} \mid s_{12}, a_{1}\right)=0.1$ e $s_{9}$ recebe o residual de 1 menos a soma das probabilidades atribuídas aos demais estados, que neste exemplo coincidentemente corresponde a $P_{\downarrow}\left(s_{9} \mid s_{12}, a_{1}\right)=0.2$.

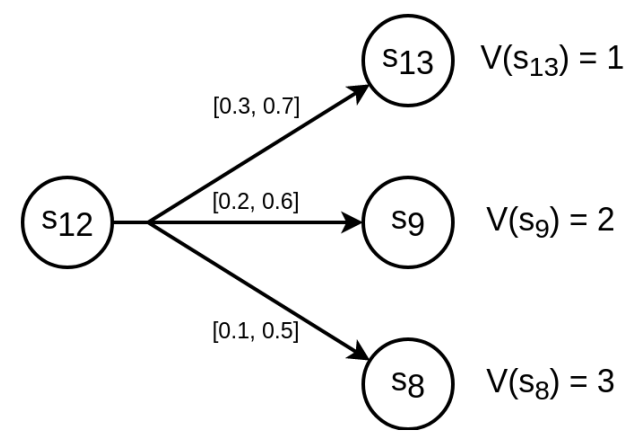

(a) Parte do BMDP da Figura 4.1

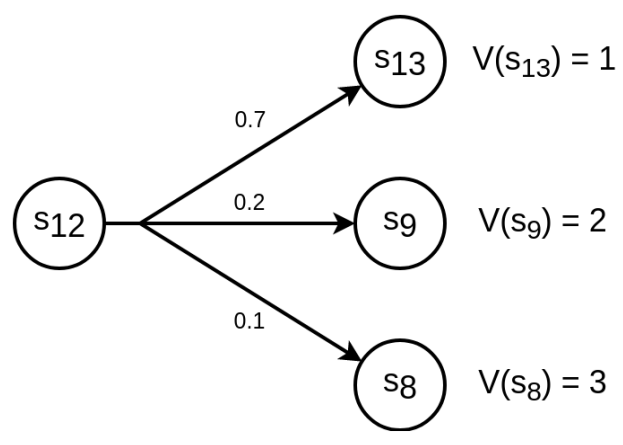

(b) Parte do MDP de maximização de ordem

Figura 4.2: Exemplo de MDP de maximização de ordem.

Dentre os MDPs $M \in X_{M_{\uparrow}}$, há dois de particular interesse para cada política $\pi$ : o MDP cuja função valor é dominada pela função valor dos demais MDPs e o MDP cuja função valor domina a dos demais MDPs.

Definição 16. MDP $\pi$-minimizante e $\pi$-maximizante. Um MDP $M$ é $\pi$-minimizante se $V^{\pi, M}$ é dominado por $V^{\pi, M^{\prime}}$ para todo MDP $M^{\prime} \in X_{M_{\mathfrak{1}}}$. Um MDP $M$ é $\pi$-maximizante se $V^{\pi, M}$ domina $V^{\pi, M^{\prime}}$ para todo MDP $M^{\prime} \in X_{M_{\uparrow}}$.

Um MDP $\pi$-minimizante corresponde à esperança inferior da recompensa acumulada desconta e um MDP $\pi$-maximizante corresponde à esperança superior da recompensa acumulada desconta com respeito ao intervalo de probabilidades [dCHQ09].

Para toda política, há pelo menos um MDP $\pi$-minimizante e um MDP $\pi$-maximizante que produzem os limites inferior e superior da função valor intervalar $V_{\uparrow}^{\pi}(s)$ para todos os estados [GLD00].

Definição 17. Função valor intervalar para uma política. A função valor intervalar de um estado $s$ para uma política $\pi$ é definida por:

$$
V_{\uparrow}^{\pi}(s)=\left[\min _{M \in X_{M_{\uparrow}}} V^{\pi, M}(s), \max _{M \in X_{M_{\uparrow}}} V^{\pi, M}(s)\right] .
$$

A Figura 4.3a) mostra um BMDP que possui apenas uma ação, portanto uma única política, chamada de política trivial, e sua função valor intervalar $V_{\uparrow}$ correspondente, considerando um fator de desconto de 0.9. O MDP $\pi$-minimizante (Figura 4.3b) e o MDP $\pi$-maximizante (figura 4.3c) para a política trivial, definem os limites inferiores e superiores da função valor intervalar deste BMDP. O MDP $\pi$-minimizante considera um MDP de maximização de ordem para a ordenação $O=s_{1}, s_{2}, s_{3}$ e o MDP $\pi$-maximizante considera um MDP de maximização de ordem para a ordenação $O=s_{3}, s_{2}, s_{1}$.

Para encontrar o MDP $\pi$-maximizante (Figura 4.3c) é utilizada a Equação 4.3. Por exemplo, considerando as transições do estado $s_{1}$ dada a única ação $a$, a probabilidade de transição intervalar 
para o estado $s_{2}$ é dada por $P_{\uparrow}\left(s_{2} \mid s_{1}, a\right)=[0.2,0.5]$, enquanto que a probabilidade intervalar para o estado $s_{3}$ é dada por $P_{\uparrow}\left(s_{3} \mid s_{1}, a\right)=[0.7,0.8]$. A probabilidade escolhida para $P\left(s_{3} \mid s_{1}, a\right)$ é 0.8 , pois é considerada a maior massa de probabilidade para o estado $s_{3}$, que está no início da ordenação $O=s_{3}, s_{2}, s_{1}$, e que cumpre a restrição da Equação 4.2. O estado $s_{2}$ fica com o limite inferior, sendo assim $P\left(s_{2} \mid s_{1}, a\right)=0.2$.

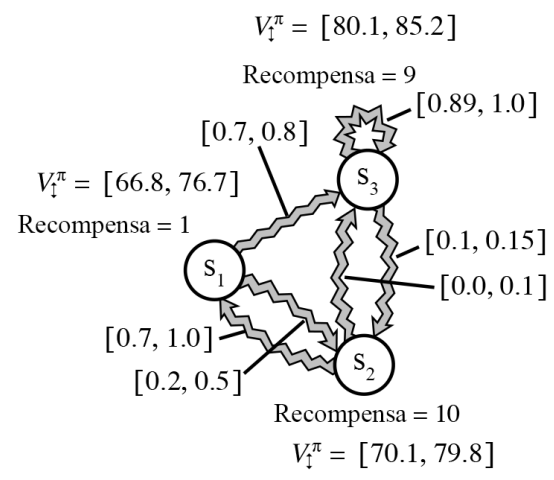

(a) $B M D P$

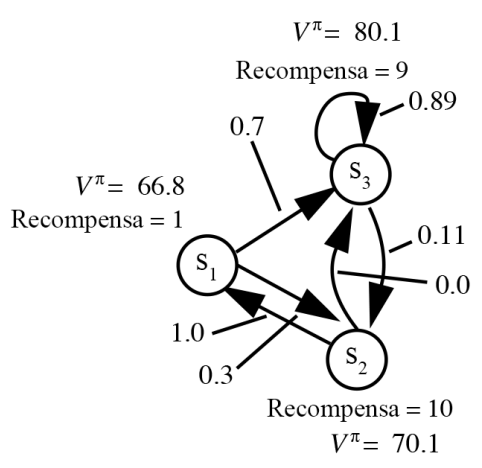

(b) $M D P \pi$-minimizante

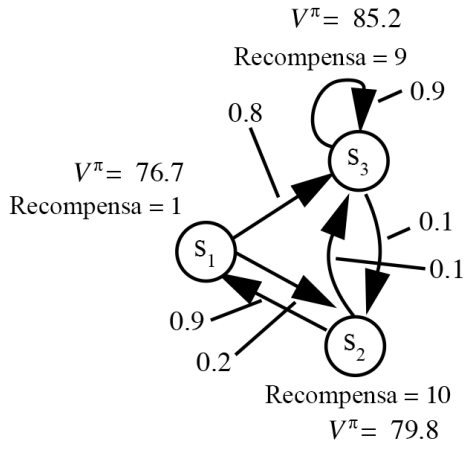

(c) $M D P \pi$-maximizante

Figura 4.3: Exemplo de BMDP e sua função valor intervalar para uma política trivial [GLD00].

A solução de um BMDP é uma política cuja função valor intervalar é ótima. Para encontrar tal política, é necessário definir como os intervalos reais podem ser comparados. Dados os intervalos $\left[l_{1}, u_{1}\right]$ e $\left[l_{2}, u_{2}\right]$, duas formas de comparação entre intervalos são definidas, as ordenações pessimista e otimista [GLD00]:

$$
\begin{aligned}
\left(\left[l_{1}, u_{1}\right] \leq_{\text {pes }}\left[l_{2}, u_{2}\right]\right) & \Leftrightarrow\left(l_{1}<l_{2} \vee\left(l_{1}=l_{2} \wedge u_{1}<u_{2}\right)\right) \\
\left(\left[l_{1}, u_{1}\right] \leq_{\text {opt }}\left[l_{2}, u_{2}\right]\right) & \Leftrightarrow\left(u_{1}<u_{2} \vee\left(u_{1}=u_{2} \wedge l_{1}<l_{2}\right)\right)
\end{aligned}
$$

A ordenação pessimista considera primeiro os limites inferiores dos intervalos em questão, e considera os limites superiores dos intervalos apenas quando os limites inferiores que estão sendo comparados forem idênticos. Analogamente, a ordenação otimista considera primeiro os limites superiores e considera os limites inferiores apenas quando os limites superiores foram idênticos.

\subsubsection{Critérios intervalares}

Assim como em MDP-IPs, também considerando a formulação baseada em Teoria dos Jogos, pode-se pensar em um BMDP como um jogo entre o agente e a natureza. Dessa forma, podese pensar em um critério em que a natureza é competitiva e um critério em que a natureza é cooperativa, porém considerando os limites da função valor intervalar, o que será chamado de critério intervalar. Dada uma natureza competitiva, o agente opta por priorizar o limite inferior em detrimento do superior, enquanto que dada uma natureza cooperativa o agente opta por priorizar o limite superior em detrimento do inferior.

Uma função valor intervalar $V_{\uparrow}^{1} \leq_{\text {pes }} V_{\uparrow}^{2}$ apenas quando $V_{\uparrow}^{1}(s) \leq_{\text {pes }} V_{\uparrow}^{2}(s)$ para todo estado $s$. Da mesma forma, $V_{\uparrow}^{1} \leq_{o p t} V_{\uparrow}^{2}$ apenas quando $V_{\uparrow}^{1}(s) \leq_{o p t} V_{\uparrow}^{2}(s)$ para todo estado $s$. Sendo assim, é possível obter duas noções de políticas ótimas.

Definição 18. Política ótima pessimista Uma política ótima pessimista $\pi^{\text {pes }}$ é uma política tal que $V_{\uparrow}^{\pi} \leq_{p e s} V_{\uparrow}^{\pi^{p e s}}$ para toda política $\pi$.

Definição 19. Política ótima otimista Uma política ótima otimista $\pi^{\text {opt }}$ é uma política tal que $V_{\uparrow}^{\pi} \leq_{o p t} V_{\uparrow}^{\pi^{o p t}}$ para toda política $\pi$. 
Dentre o conjunto de políticas, há pelo menos uma política que atinge o valor máximo para cada ordenação apresentada, i.e., há pelo menos uma política ótima pessimista e uma política ótima otimista, que são associadas às funções valor intervalares ótimas pessimistas e otimistas, respectivamente.

Definição 20. Função valor intervalar ótima pessimista Uma função valor intervalar ótima pessimista é definida por: $V_{\uparrow}^{\text {pes }}=\max _{\pi \in \Pi}\left(V_{\uparrow}^{\pi}\right)$ considerando $\leq_{\text {pes }}$ para ordenar as funções valores intervalares.

Definição 21. Função valor intervalar ótima otimista Uma função valor intervalar ótima pessimista é definida por: $V_{\uparrow}^{\text {opt }}=\max _{\pi \in \Pi}\left(V_{\uparrow}^{\pi}\right)$ considerando $\leq_{\text {opt }}$ para ordenar as funções valores intervalares.

Entende-se por função valor intervalar ótima otimista o melhor que o agente pode obter se a natureza for cooperativa com ele, enquanto que a função valor intervalar ótima pessimista representa o melhor que o agente pode obter considerando uma situação adversarial.

A função valor intervalar de um BMDP é obtida pelo cálculo dos limites inferior e superior de forma separada, de modo que para cada limite é necessário encontrar o MDP de maximização de ordem correspondente. Para isso, define-se a função qualidade mínima e máxima para BMDPs considerando uma dada função valor.

Definição 22. Função qualidade mínima. A função qualidade mínima para o estado $s$ e ação $a$ para uma dada função valor $V$, considera o MDP de maximização de ordem $M$ para uma ordenação dos estados em ordem crescente de $V$ :

$$
Q_{\min }^{V}(s, a)=\min _{M \in X_{M_{\mathfrak{1}}}}\left\{R(s, a)+\gamma \sum_{s^{\prime} \in S} P^{M}\left(s^{\prime} \mid s, a\right) V\left(s^{\prime}\right)\right\} .
$$

Definição 23. Função qualidade máxima. A função qualidade máxima para o estado $s$ e ação $a$ para uma dada função valor $V$, considera o MDP de maximização de ordem $M$ para uma ordenação dos estados em ordem decrescente de $V$ :

$$
Q_{\text {max }}^{V}(s, a)=\max _{M \in X_{M_{\uparrow}}}\left\{R(s, a)+\gamma \sum_{s^{\prime} \in S} P^{M}\left(s^{\prime} \mid s, a\right) V\left(s^{\prime}\right)\right\} .
$$

Com base nas funções qualidade mínima e máxima, os operadores $T_{p e s}$ e $T_{\text {opt }}$ são definidos pela aplicação da Equação de Bellman para cada um dos critérios intervalares, considerando cada limite do intervalo separadamente.

$$
\begin{aligned}
& \left(T_{\text {pes }} V_{\uparrow}\right)(s)=\max _{a \in A, \leq_{\text {pes }}}\left[Q_{\text {min }}^{V_{\downarrow}}(s, a), Q_{\text {max }}^{V_{\uparrow}}(s, a)\right] \\
& \left(T_{\text {opt }} V_{\uparrow}\right)(s)=\max _{a \in A, \leq_{\text {opt }}}\left[Q_{\text {min }}^{V_{\downarrow}}(s, a), Q_{\text {max }}^{V_{\uparrow}}(s, a)\right]
\end{aligned}
$$

Dados os operadores $T_{p e s}$ e $T_{o p t}$, o limite inferior da função valor intervalar é calculado utilizando apenas o limite inferior $V_{\downarrow}$ da função valor intervalar $V_{\uparrow}$, enquanto que para o cálculo do limite superior é considerado apenas o limite superior $V_{\uparrow}$ de $V_{\uparrow}$. Os pontos fixos de cada operador são respectivamente as funções valor intervalares ótimas $V_{\uparrow}^{\text {pes }}$ e $V_{\uparrow}^{\text {opt }}$. Assim, as equações de Bellman adaptadas para BMDPs, segundo cada critério intervalar, são definidas por [GLD00]:

$$
V_{\uparrow}^{\text {pes }}(s)=\max _{a \in A, \leq \text { pes }}\left[Q_{\text {min }}^{V_{\downarrow}^{\text {pes }}}(s, a), Q_{\max }^{V_{\uparrow}^{\text {pes }}}(s, a)\right],
$$

e

$$
V_{\uparrow}^{o p t}(s)=\max _{a \in A, \leq \text { opt }}\left[Q_{\text {min }}^{V_{\downarrow}^{o p t}}(s, a), Q_{\max }^{V_{\uparrow}^{o p t}}(s, a)\right] .
$$

O limite inferior $V_{\downarrow}^{\text {pes }}$ de $V_{\uparrow}^{\text {pes }}(s)$ representa o melhor que o agente pode obter se a natureza é um adversário que tenta minimizar a função valor resultante ao selecionar o MDP que minimiza 
$V^{\pi, M}$, isto é o limite inferior $V_{\downarrow}^{\text {pes }}$ corresponde ao critério maximin para MDP-IPs. Note que, $V_{\uparrow}^{\text {pes }}$ corresponde a quão bem o agente pode se sair, caso a natureza coopere com ele. O limite superior $V_{\uparrow}^{\text {opt }}$ de $V_{\uparrow}^{\text {opt }}(s)$ representa o melhor que o agente pode obter caso a natureza seja cooperativa com ele, enquanto que o limite inferior $V_{\downarrow}^{\text {opt }}$ representa quão mal o agente pode se sair se a natureza o trair. Note que, $V_{\uparrow}^{\text {opt }}$ corresponde ao critério maximax para MDP-IPs.

\subsubsection{Critério robusto}

Partindo do pressuposto que a natureza é racional e que sempre escolherá o MDP que maximiza o seu comportamento dada a ação escolhida por um agente também racional, uma outra forma de pensar sobre critério de otimização de um BMDP consiste em considerar apenas um dos limites do intervalo de uma função valor intervalar, isto é, considerar apenas o critério maximin ou maximax. Neste trabalho é considerado apenas o critério maximin, também chamado de critério robusto.

O critério robusto para BMDPs, equivalente ao critério robusto para MDP-IPs. Assim, basta utilizar uma função valor $V(s)$ para todo estado, ao invés de uma função valor intervalar $V_{\uparrow}(s)$.

Seja $L$ um operador robusto sobre $\mathrm{V}(\mathrm{s})$, dado por:

$$
(L V)(s)=\max _{a \in A}\left\{Q_{\min }^{V}(s, a)\right\} .
$$

O ponto fixo de $L$ é a função valor robusta ótima $V^{*}(s)$. O princípio de otimalidade para MDP-IPs adaptado para BMDPs e considerando o critério robusto é dado por:

$$
V^{*}(s)=\max _{a \in A}\left\{Q_{\min }^{V^{*}}(s, a)\right\},
$$

A política ótima robusta $\pi^{*}$ é definida como:

$$
\pi^{*}(s)=\underset{a \in A}{\operatorname{argmax}}\left\{Q_{\min }^{V^{*}}(s, a)\right\} .
$$

\subsection{Algoritmos}

Em Givan et al (2000), foram propostos os algoritmos de Avaliação de Política Intervalar e Iteração de Valor Intervalar (IVI) para BMDPs de horizonte infinito.

Neste seção são apresentados o algoritmo que calcula a função Q para BMDPs, o algoritmo de Iteração de Valor Intervalar e o algoritmo de Iteração de Política Robusta para BMDPs de horizonte infinito. Outros algoritmos para BMDP com outros horizontes são apresentados na Seção 7.1 do capítulo de Trabalhos Correlatos.

\subsubsection{Cálculo da função Q para BMDPs}

Uma função comum a todos os algoritmos para BMDP é aquela que gera um MDP de maximização de ordem para calcular $Q_{\min }^{V}$ ou $Q_{\max }^{V}$ (Equações 4.6 e 4.7). No entanto, o custo para calcular a função qualidade é menos dispendioso do que em MDP-IPs, uma vez que não é necessário utilizar um solver para determinar as probabilidades de transição [DdBCS11b, DSdB11b, SdBC09]; basta utilizar uma estratégia gulosa para encontrar o MDP de maximização de ordem correspondente à entrada do algoritmo.

O algoritmo QVAlueBMDP (Algoritmo 1) calcula e retorna a função qualidade de um par estado-ação para um BMDP considerando uma tipo de ordenação e uma função valor. A entrada do algoritmo é um BMDP $B$, uma função valor $V$, um par estado-ação $(s, a)$ e um tipo de ordenação order, que pode ser crescente ou decrescente. Na linha 2, são obtidas as probabilidades de transição retornadas pelo algoritmo OrderMAXimizingMDP. Assim, a qualidade é calcula pela soma da recompensa imediata de executar a ação $a$ no estado $s$ (linha 3 ) com o valor esperado dos estados sucessores (linhas 5 e 6). O resultado é retornado na linha 7. 

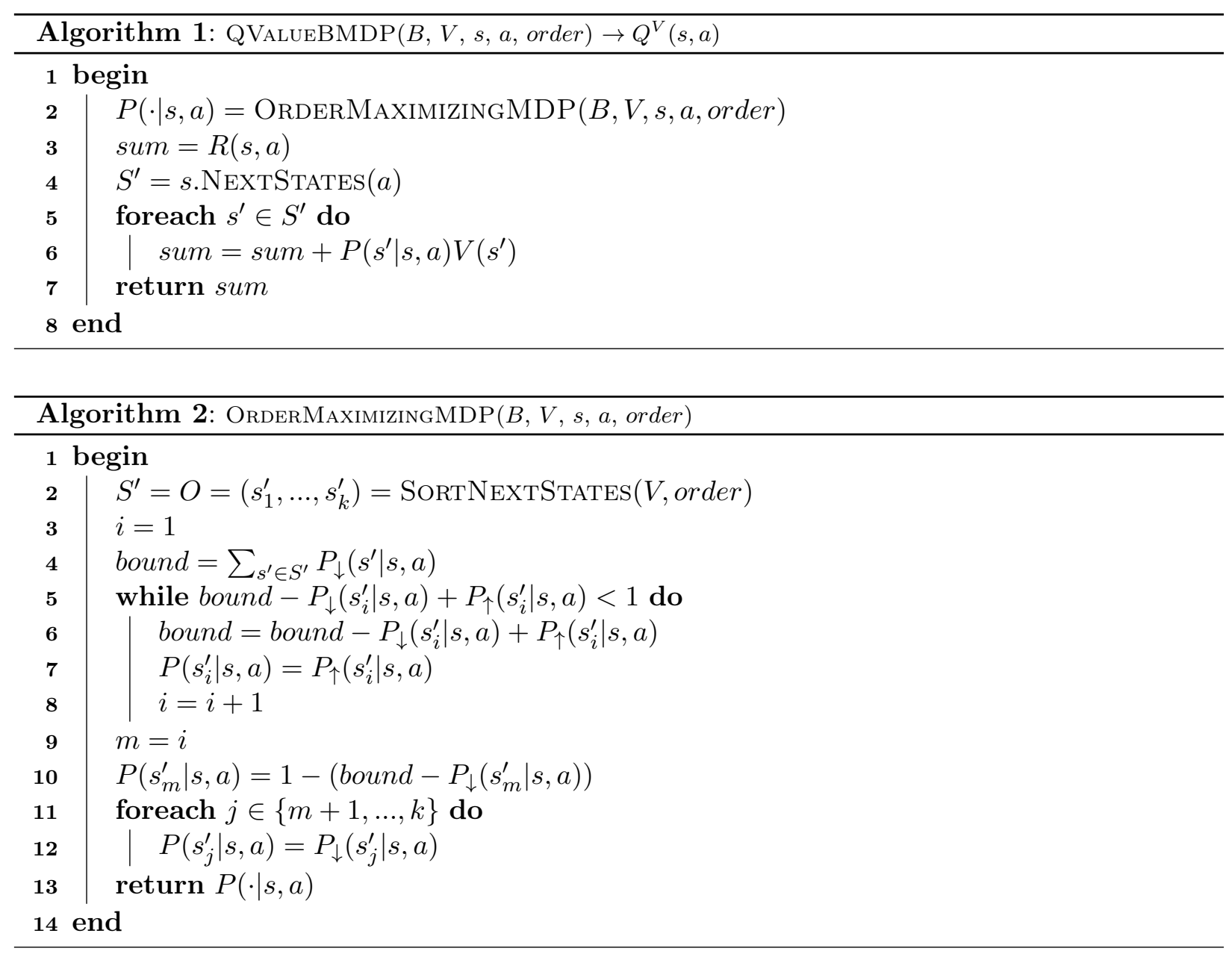

O algoritmo OrderMaximizingMDP (Algoritmo 2) recebe um BMDP $B$, uma função valor $V$, um par estado-ação $(s, a)$ e um tipo de ordenação order (que pode ser crescente ou decrescente), e retorna as probabilidades de transição de $(s, a)$ referentes ao MDP de maximização de ordem $M_{O}$ obtido pela ordenação dos estados segundo o tipo de ordenação order levando em conta os valores de $V$. Na linha 2, a função SortNextStates ordena os estados de acordo com a função valor $V$ e o tipo de ordenação order e retorna a ordenação de estados $O$, que também será chamada de $S^{\prime}$. Uma vez obtida a ordenação $O$, as probabilidades de transição de $(s, a)$ são atribuídas de acordo com a Equação 4.3. Na Linha 4, a variável bound é inicializada com a soma dos limites inferiores do intervalo. As Linhas 5 a 8 atualizam a probabilidade $P\left(s_{i}^{\prime} \mid s, a\right)$ com o limite superior $P_{\uparrow}\left(s_{i}^{\prime} \mid s, a\right)$ para cada estado $s_{i}^{\prime}$ tal que $i<m$. Na linha 10 a probabilidade $P\left(s_{m}^{\prime} \mid s, a\right)$ é atualizada com o valor residual para garantir que a soma das probabilidades para todos os próximos estados seja igual a 1 . As Linhas 11 e 12 atualizam as probabilidades $P\left(s_{j}^{\prime} \mid s, a\right)$ com o limite inferior $P_{\downarrow}\left(s_{j}^{\prime} \mid s, a\right)$, para $m<j \leq k$. Finalmente, o algoritmo OrderMAXIMIZINGMDP retorna a probabilidade de transição $P(\cdot \mid s, a)$ referente ao MDP de maximização de ordem $M_{O}$ na Linha 13.

\subsubsection{Iteração de Valor Intervalar}

O algoritmo de Iteração de Valor Intervalar (Interval Value Iteration - IVI) depende da forma como os intervalos reais são comparados. Assim, existem duas versões do IVI, a versão otimista $\mathrm{IVI}_{\text {opt }}$ e a pessimista $\mathrm{IVI}_{\text {pes }}$.

A essência do algoritmo IVI é a mesma do algoritmo VI. IVI recebe uma função valor intervalar inicial $V_{\uparrow}^{0}$ e a cada iteração calcula $V_{\uparrow}^{t+1}=\left[V_{\downarrow}^{t+1}(s), V_{\uparrow}^{t+1}(s)\right]$ com base na função valor intervalar $V_{\uparrow}^{t}$. A execução do IVI termina quando ambos os limites são menores que um erro $\epsilon$ para todos os estados. As diferenças se dão na necessidade de atribuição das probabilidades precisas para as 
transições de estado de cada par estado-ação e no cálculo dos limites da função valor intervalar. Ambos os cálculos são realizados separadamente para cada intervalo.

No algoritmo $\mathrm{IVI}_{\text {pes }}$, primeiro os limites inferiores da função valor intervalar $V_{\downarrow}^{t+1}$ são atualizados com base no limite inferior da função valor intervalar $V_{\downarrow}^{t}$ usando a seguinte equação:

$$
V_{\downarrow}^{t+1}(s)=\max _{a \in A}\left\{Q_{\text {min }}^{V_{\downarrow}^{t}}(s, a)\right\} .
$$

Para cada estado, todas as ações $\sigma_{\downarrow}^{t}$ que produzem esse valor máximo do limite inferior são armazenadas para serem usadas no cálculo da atualização do limite superior:

$$
\sigma_{\downarrow}^{V_{\downarrow}^{t}}(s)=\underset{a \in A}{\operatorname{argmax}}\left[Q_{\min }^{V_{\downarrow}^{t}}(s, a)\right] .
$$

Depois o limite superior $V_{\uparrow}^{t+1}$ é atualizado considerando o limite superior $V_{\uparrow}^{t}$ e apenas as ações $\sigma_{\downarrow} V_{\downarrow}^{t}$ :

$$
V_{\uparrow}^{t+1}(s)=\max _{a \in \sigma_{\downarrow}^{t}(s)}\left\{Q_{\max }^{V_{\uparrow}^{t}}(s, a)\right\} .
$$

A versão otimista do algoritmo de Iteração de Valor Intervalar, $\mathrm{IVI}_{\text {opt }}$, é resolvida de maneira similar, mas atualizando primeiro o limite superior:

$$
V_{\uparrow}^{t+1}(s)=\max _{a \in A}\left\{Q_{\max }^{V_{\uparrow}^{t}}(s, a)\right\},
$$

e depois, calculando o conjunto de ações $\rho^{V_{\downarrow}^{t}}(s)$ que produz esse limite superior:

$$
\rho^{V_{\uparrow}^{t}}(s)=\underset{a \in A}{\operatorname{argmax}}\left[Q_{\max }^{V_{\uparrow}^{t}}(s, a)\right] .
$$

Considerando apenas esse último conjunto de ações, o limite inferior do intervalo é atualizado:

$$
V_{\downarrow}^{t+1}(s)=\max _{a \in \rho V_{\uparrow}^{t}(s)}\left\{Q_{\text {min }}^{V_{\downarrow}^{t}}(s, a)\right\} .
$$

\subsubsection{Iteração de Política Robusto}

O algoritmo de Iteração de Política Robusto para BMDPs é baseado no algoritmo de Iteração de Política Robusto para MDP-IPs. A principal diferença dos algoritmos é que a versão para BMDPs consegue calcular as probabilidades de forma gulosa, o que torna o algoritmo mais eficiente em relação à sua versão para MDP-IPs.

O algoritmo iterativamente melhora a política ao aplicar os passos de avaliação de política robusta e melhoria de política robusta para todos os estados em $S$. Em ambos os passos, o algoritmo 1 é executado para calcular $Q_{m i n}^{V^{t}}$, isto é usando o valor crescente para o parâmetro order.

Avaliação de política robusta. Calcula a função valor de uma dada política na iteração $t$, denotada por $\pi^{t}$, considerando um agente competitivo:

$$
V^{t}(s)=Q_{\min }^{V^{t}}\left(s, \pi^{t}(s)\right)
$$

Melhoria de política robusta. Atualiza a política com a melhor ação sob uma situação competitiva utilizando a função valor $V^{t}$ calculada no passo anterior:

$$
\pi^{t+1}(s)=\underset{a \in A}{\operatorname{argmax}}\left\{Q_{\text {min }}^{V^{t}}(s, a)\right\} .
$$


É possível também implementar uma versão otimista do algoritmo de iteração de política. 


\section{Capítulo 5}

\section{Programação assíncrona para BMDPs}

Givan et al. (2000) propuseram os algoritmos síncronos de Iteração de Valor Intervalar e Avaliação de Política Intervalar para resolução de BMDPs de horizonte infinito. Por serem algoritmos síncronos, ambos podem ser ineficientes por atualizar o conjunto completo de estados a cada iteração. Bertsekas e Yu (2010) propuseram um algoritmo de Iteração de Política Assíncrono para resolução de várias classes de MDPs, incluindo MDP-IPs, mas sem especificar formas de particionar os estados. Dai et al. (2007) propuseram o algoritmo de Iteração de Valor Topológico para resolver eficientemente MDPs considerando componentes fortemente conexos.

Neste capítulo são apresentadas as contribuições teóricas deste trabalho, que incluem a proposição de algoritmos assíncronos baseados em Iteração de Política Robusto para a resolução de BMDPs de horizonte infinito, cuja finalidade é obter políticas ótimas completas de maneira mais eficiente em termos de tempo de execução quando comparados aos algoritmos síncronos existentes. Os algoritmos propostos utilizam o método guloso para seleção de probabilidades assim como proposto por Givan et al (2000) e os modelos de particionamento do conjunto de estados assim como proposto por Bertsekas e Yu (2010) e Dai et al (2007).

Entre os algoritmos propostos estão RAPI e RTPI que fazem o particionamento do conjunto de estados a fim de reduzir, em relação ao algoritmo RPI, o número de atualizações e o tempo de execução até a convergência. O algoritmo RAPI particiona o conjunto de estados $S$ em subconjuntos de estados $S_{i}$, definidos aleatoriamente. Cada subconjunto é atualizado sequencialmente com um único processador ou paralelamente em um sistema multiprocessador até a convergência parcial de cada subconjunto. Este processo é repetido até a convergência da política completa (Figura 5.1a). Uma vez que o particionamento é aleatório, passa a ser importante o tamanho dos subconjuntos. O algoritmo RTPI particiona o conjunto de estados em componentes fortemente conexos, que são atualizados em ordem topológica reversa (Figura 5.1b). O algoritmo RTPI converge mediante a atualização de apenas uma meta-atualização para cada subconjunto $S_{i}$. Nesta seção também são propostas heurísticas de inicialização da função valor para BMDPs.

Este capítulo é organizado como segue. Na seção 5.1 são apresentados os operadores conservativos necessários para a convergência de alguns dos algoritmos propostos. Na Seção 5.2 são abordadas formas alternativas para inicialização dos algoritmos. Nas seções 5.3 e 5.4 são apresentados os algoritmos RAPI e RTPI, respectivamente.

\subsection{Operadores de atualização conservativos robustos}

Como os algoritmos assíncronos para MDPs (Seção 2.2.2), os algoritmos assíncronos propostos para resolver BMDPs (exceto o algoritmo RTPI) precisam dos operadores conservativos que devem ser aplicados a cada subconjunto $S_{i} \subseteq S$, mas considerando uma minimização extra com relação as probabilidades de transição. Portanto, as Equações 2.12, 2.13 e 2.14 são modificadas, gerando os operadores de melhoria de política conservativa robusta e avaliação de política conservativa robusta. 


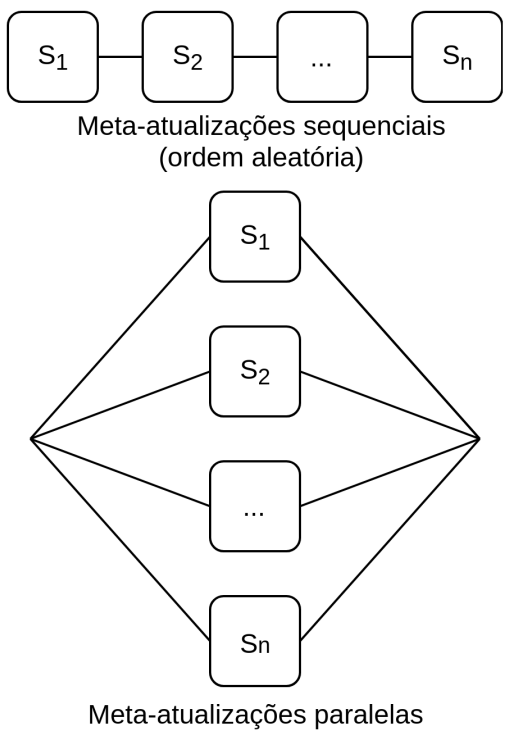

(a) Particionamento aleatório

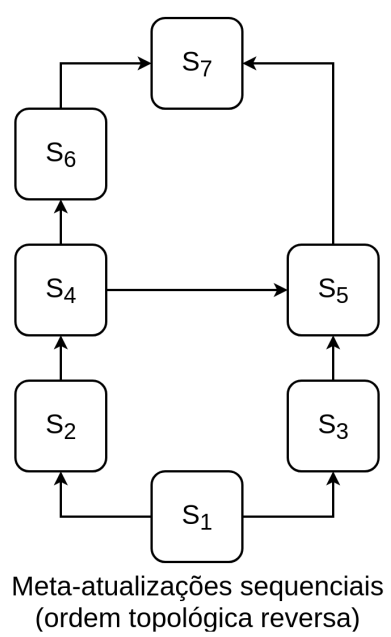

(b) Particionamento baseado em SCCs

Figura 5.1: Duas formas de particionamento do algoritmo de Iteração de Política Assíncrono.

Melhoria de política conservativa robusta. Atualiza a política $\pi^{t+1}$ e função valor $V^{t+1}$ baseada na função valor $V^{t}$ para todo estado $s \in S_{i}$ :

$$
\begin{gathered}
\pi^{t+1}(s)=\underset{a \in A}{\operatorname{argmax}}\left\{Q_{\min }^{V^{t}}(s, a)\right\}=\underset{a \in A}{\operatorname{argmax}}\left\{\min _{M \in X_{M_{\downarrow}}}\left\{R(s, a)+\gamma \sum_{s^{\prime} \in S} P^{M}\left(s^{\prime} \mid s, a\right) V^{t}\left(s^{\prime}\right)\right\}\right\}, \\
V^{t+1}(s)=\max _{a \in A}\left\{Q_{\min }^{V^{t}}(s, a)\right\}=\max _{a \in A}\left\{\min _{M \in X_{M_{\uparrow}}}\left\{R(s, a)+\gamma \sum_{s^{\prime} \in S} P^{M}\left(s^{\prime} \mid s, a\right) V^{t}\left(s^{\prime}\right)\right\}\right\},
\end{gathered}
$$

e guarda $V^{t+1}$ na variável auxiliar $V_{\text {aux }}^{t+1}$ para ser usada na avaliação de política conservativa.

Avaliação de política conservativa robusta. Para garantir que a função valor da política $\pi^{t+1}$ nunca decresça, a seguinte atualização é executada para todo estado $s \in S_{i}$ :

$$
V^{t+1}(s)=\max \left\{V_{a u x}^{t+1}(s), \min _{M \in X_{M_{\mathfrak{1}}}}\left\{R\left(s, \pi^{t+1}(s)\right)+\gamma \sum_{s^{\prime} \in S} P^{M}\left(s^{\prime} \mid s, \pi^{t+1}(s)\right) V^{t+1}\left(s^{\prime}\right)\right\}\right\} .
$$

Os algoritmos 3 e 4 implementam as Equações 5.1 e 5.2, e 5.3, respectivamente. Eles trabalham com um subconjunto $S_{i} \subseteq S$ e chamam o algoritmo QVALuEBMDP (Algoritmo 1) para calcular $Q_{\text {min }}^{V}(s, a)$ para cada par estado-ação.

O algoritmo RunPISubSet (Algoritmo 5) é uma versão modificada do algoritmo síncrono RPI, que executa uma meta-atualização em um subconjunto $S_{i}$. RunPISUBSET faz chamadas aos operadores de avaliação e melhoria de política conservativos sobre $S_{i} \subseteq S$ a cada iteração até que não haja mudança na política parcial. O operador de avaliação de política conservativo atualiza a função valor dos estados $s \in S_{i}$, representada por $V_{i}$. O operador de melhoria de política conservativo atualiza a política e funções valor dos estados $s \in S_{i}$, representados por $\pi_{i}, V_{i}$ e $V_{\text {auxi }}$. Note que para ambos os passos, a função valor de todo o espaço de estados deve estar disponível. 

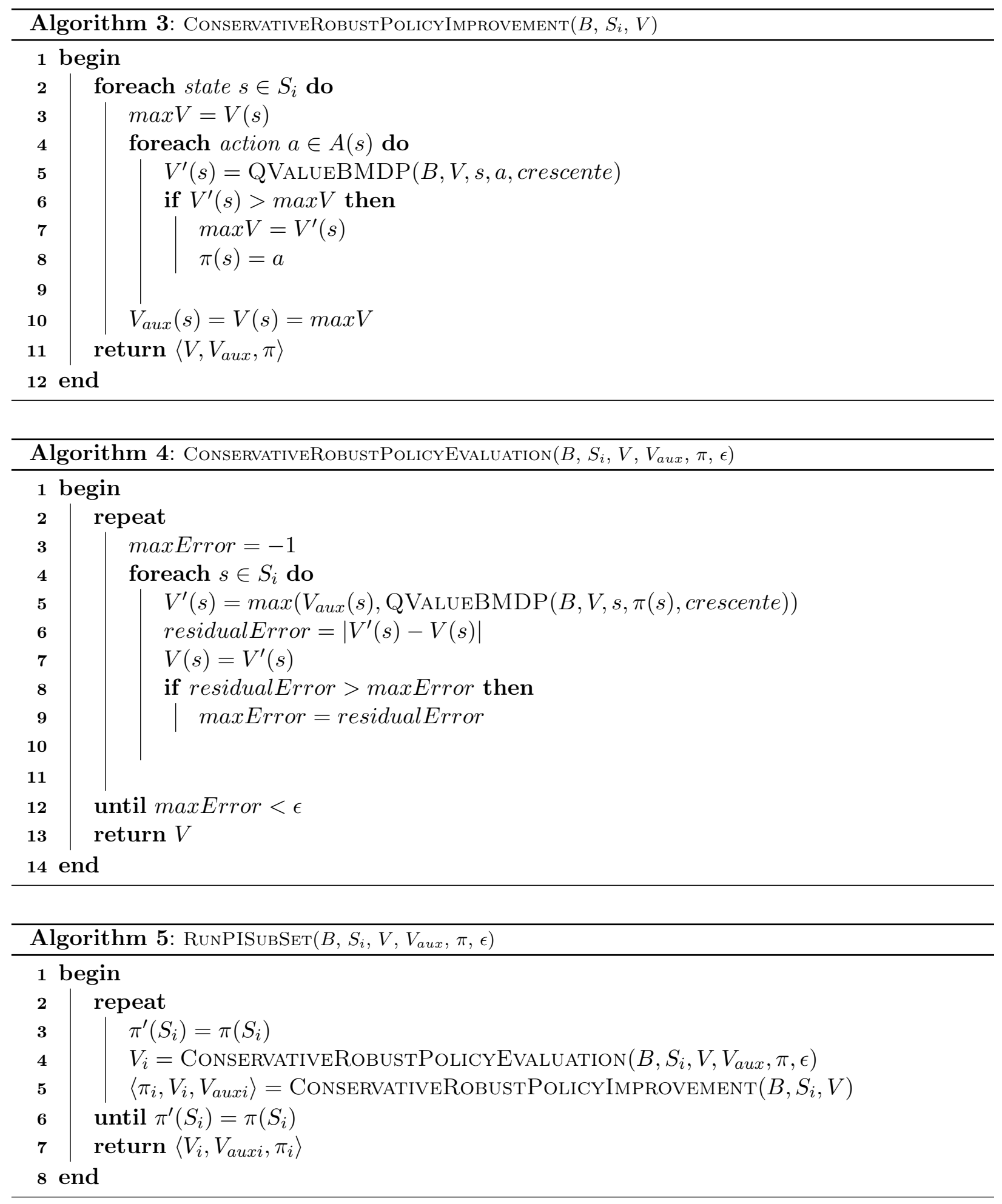

\subsection{Política Inicial e Função Valor Inicial}

O algoritmo de Iteração de Política, que utiliza um sistema de equações lineares para o passo de avaliação de política, precisa apenas de uma política inicial como entrada. Já o algoritmo assíncrono de Iteração de Política Modificado, que utiliza os operadores de atualização conservativos, precisa que ambas as funções valor $V^{0}$ e $V_{a u x}^{0}$, e a política inicial $\pi^{0}$ sejam inicializadas. Note que $V^{0}$ e $V_{a u x}^{0}$ podem ser inicializados com o mesmo valor.

Dado um BMDP $B$, uma inicialização adequada da política $\pi^{0}$ e função valor $V^{0}$ pode diminuir o número de atualizações necessárias até a convergência e, consequentemente, o tempo de execução 


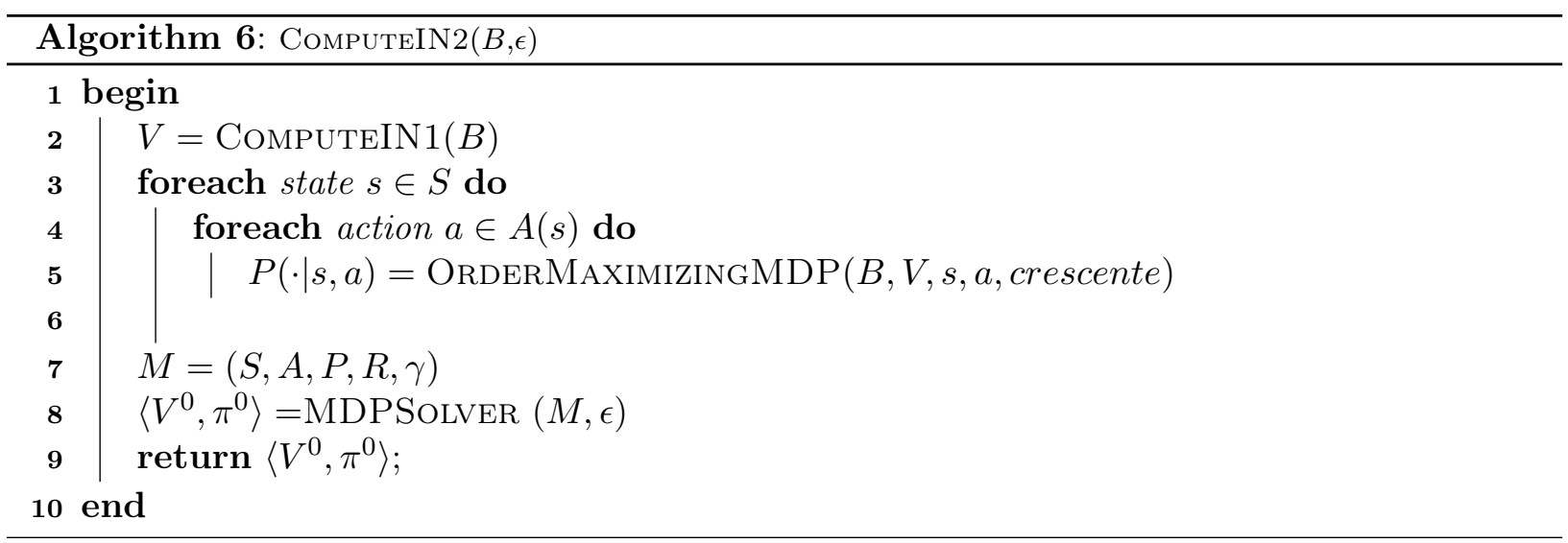

do algoritmo. Com esse intuito, são propostos três métodos heurísticos para inicializar os pares $\left(\pi^{0}(s), V^{0}(s)\right)$, chamados IN0, IN1 e IN2:

(IN0) $V^{0}(s)=h_{0}(s)=0$ e $\pi^{0}(s)$ aleatória;

(IN1) $V^{0}(s)=h_{\text {rmax }}(s)=\max _{a}\{|R(s, a)|\} /(1-\gamma)$ e $\pi^{0}(s)=\operatorname{argmax}_{a}\{R(s, a)\} /(1-\gamma)$; e

(IN2) $V^{0}(s)=h_{\mathrm{MDP}}(s)=V^{*}(s)$ e $\pi^{0}(s)=\operatorname{argmax}_{a} V^{*}(s)$, sendo $V^{*}(s)$ a função valor ótima de um MDP $M$ criado a partir do BMDP $B$.

O método de inicialização IN0 é um método trivial que inicializa os valores com 0 e a política aleatoriamente. IN1 inicializa a função valor de um estado com a recompensa acumulada máxima possível, enquanto que a política inicial para este estado corresponde a ação que produz esta recompensa acumulada máxima. O método IN2, por sua vez, resolve um MDP $M$ criado a partir do BMDP $B$.

O algoritmo COMPUTE $h_{M D P}$ (Algoritmo 6) implementa o método de inicialização IN2, através da resolução de um MDP $M$ de maximização de ordem criado a partir do BMDP $B$ com uma ordenação crescente da função valor, o que é feito chamando a função OrderMAXIMIzINGMDP e que: (i) garante escolher uma probabilidade $P(\cdot \mid s, a)$ tal que $\sum_{s^{\prime} \in S} P\left(s^{\prime} \mid s, a\right)=1$ para todo par estado-ação; e (ii) concorda com o critério de maximização usado para BMDPs. O parâmetro $V$ da função OrDERMAXIMIZINGMDP pode ser inicializada por uma heurística (Linha 2), por exemplo, $V=h_{r \max }(\mathrm{IN} 1)$ ou $V=h_{0}$ (IN0). Nas Linha 3 a 7 , é gerado o MDP de maximização de ordem $M$. Na Linha 8, é calculada a função valor ótima e política ótima do MDP $M$ utilizando qualquer algoritmo para MDPs de horizonte infinito, inclusive algoritmos aproximados.

\subsection{RAPI}

Bertsekas e Yu [BY10a] propuseram um algoritmo geral de Iteração de Política Assíncrono (API) para resolver tanto MDPs como algumas de suas extensões, como MDP-IPs. No entanto, não foi discutido como fazer o particionamento do conjunto de estados e como diferentes formas de particionamento podem afetar a eficiência dos algoritmos. Assim, neste trabalho é proposto o algoritmo de Iteração de Política Assíncrono Robusto (Robust Asynchronous Policy Iteration RAPI) [RDdB16, RdBD19], uma adaptação do algoritmo API para BMDPs com particionamento aleatório em versões sequencial e paralela.

O algoritmo RAPI particiona o conjunto de estados $S$ de um BMDP $B$ em subconjuntos disjuntos não vazios de forma aleatória. Para cada $S_{i} \subseteq S$, a função valor $V(s)$ e a política $\pi(s)$ são atualizadas de maneira assíncrona (seja usando um sistema multiprocessador ou com único processador, em paralelo ou sequencialmente). Note que, quando se utiliza computação paralela, a função valor e política para cada estado $s \in S$ estão disponíveis para todos os subconjuntos, mas não estão sempre atualizadas, devido a um atraso na troca de informação entre os processadores. 


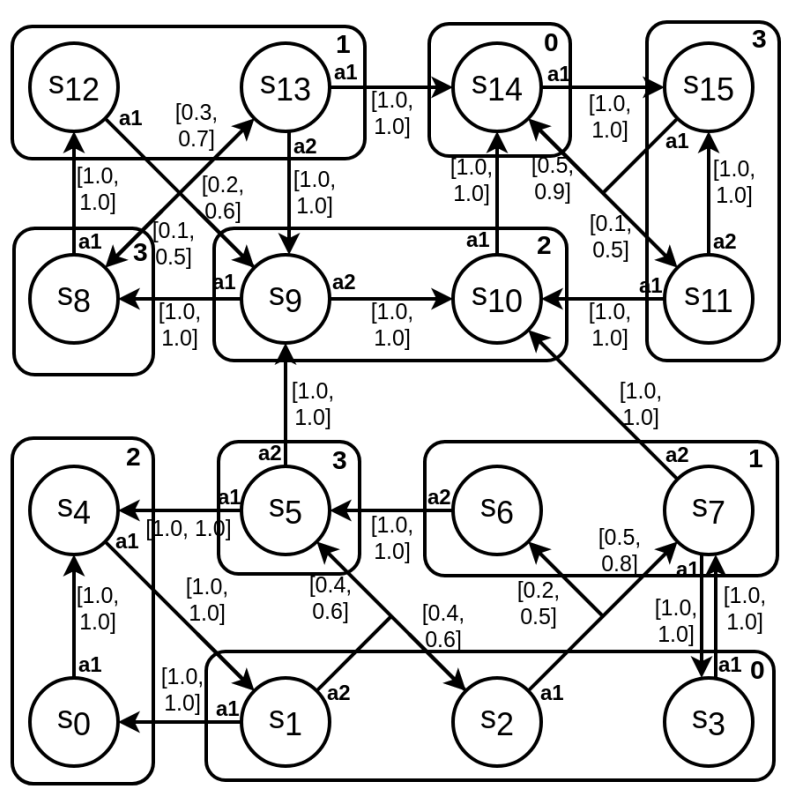

(a) Particionado aleatoriamente

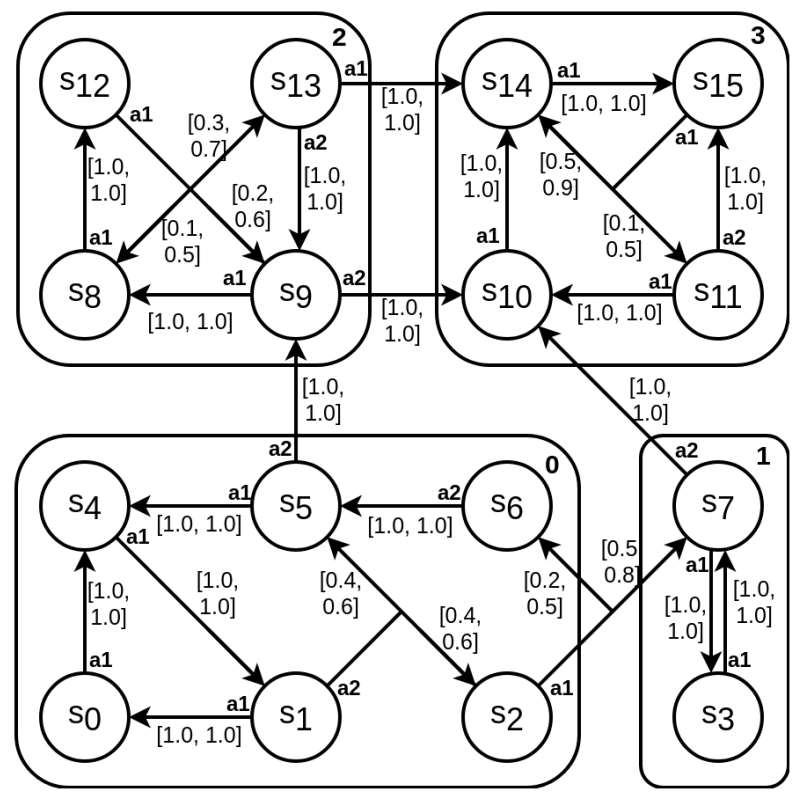

(b) Particionado topologicamente

Figura 5.2: BMDP da Figura 4.1 particionado de formas diferentes. (a) Particionamento aleatório; (b) Particionamento topológico.

O pseudo-código do algoritmo RAPI é mostrado no Algoritmo 7. Na Linha 2, a política e a função valor podem ser inicializadas com qualquer dos métodos heurísticos descritos na subseção 5.2, i.e. IN0, IN1 e IN2. Na linha 3 o algoritmo particiona aleatoriamente o conjunto de estados em $Z=\left\{S_{1}, \ldots, S_{n}\right\}$, em que $\alpha$ é o número de estados em cada subconjunto. A Figura 5.2a mostra um BMDP, em que os estados dentro de um retângulo arredondado com um mesmo número estão no mesmo subconjunto de um particionamento aleatório.

No Algoritmo 7, o algoritmo RUNPISUBSET pode ser chamado sequencialmente ou em paralelo (de acordo com o parâmetro exec). Na versão sequencial do RAPI, denotada por RAPI-sequential, a função RunPISUBSET é chamada para cada $S_{i}$ e a ordem em que os subconjuntos são atualizados é arbitrária.

Na versão paralela do RAPI, chamada de RAPI-parallel, cada processador $i$ tem a responsabilidade de atualizar a função valor $V(s)$ e a política $\pi(s)$, para todo $s \in S_{i}$, tendo sempre acesso a $V(s)$ e $\pi(s)$, para todo $s \in S$. Threads são criadas para cada subconjunto disjunto não vazio $S_{i} \subseteq S$ e a agenda (schedule) de execução é definida pelo sistema operacional. Note que a cada estágio que a função valor ou a política são atualizadas, todos os processadores tem acesso a essa informação.

Quando a política parcial de um subconjunto converge, a thread correspondente espera a convergência dos outros subconjuntos (que podem ter algum atraso). Note que os valores podem ser lidos por todos os subconjuntos, mas podem ser escritos apenas pelas threads responsáveis pelo subconjunto $S_{i}$, garantindo que não há concorrência durante as atualizações.

Na prática, o algoritmo RAPI deve parar depois de um número finito de passos. Isto é feito ao executar no final de cada iteração, uma avaliação de política robusta conservativa e uma melhoria de política robusta conservativa para o conjunto completo de estados (Linhas 15-16). Este processo é executado até que a política completa convirja. O algoritmo RAPI converge (Proposição 2 e Teorema 4).

Proposição 2. Para cada BMDP de horizonte infinito vale a seguinte propriedade: (i) a função $Q_{\min }^{V}$ (Equação 4.6) é monótona, i.e., se $V(s) \leq V^{\prime}(s)$ para todo $s \in S$ então $Q_{\min }^{V}(s, a) \leq Q_{\min }^{V^{\prime}}(s, a)$ para todo $s \in S$ e $a \in A$; (ii) o operador $L$ (Equação 4.12) tem um único ponto fixo $V^{*}$; (iii) existem duas funções $\underline{V}$ and $\bar{V}$ tais que $\underline{V} \leq V \leq \bar{V}, \underline{V} \leq L \underline{V} \leq L \bar{V} \leq \bar{V}$ e $\lim _{k \rightarrow \infty}\left(\left(L^{k} \underline{V}\right)(s)\right)=$ $\lim _{k \rightarrow \infty}\left(\left(L^{k} \bar{V}\right)(s)\right)=V^{*}(s), \forall s \in S$, em que $L^{k}$ é um composição do operador $L$, i.e. $\left(L^{k} V\right)(s)=$ 


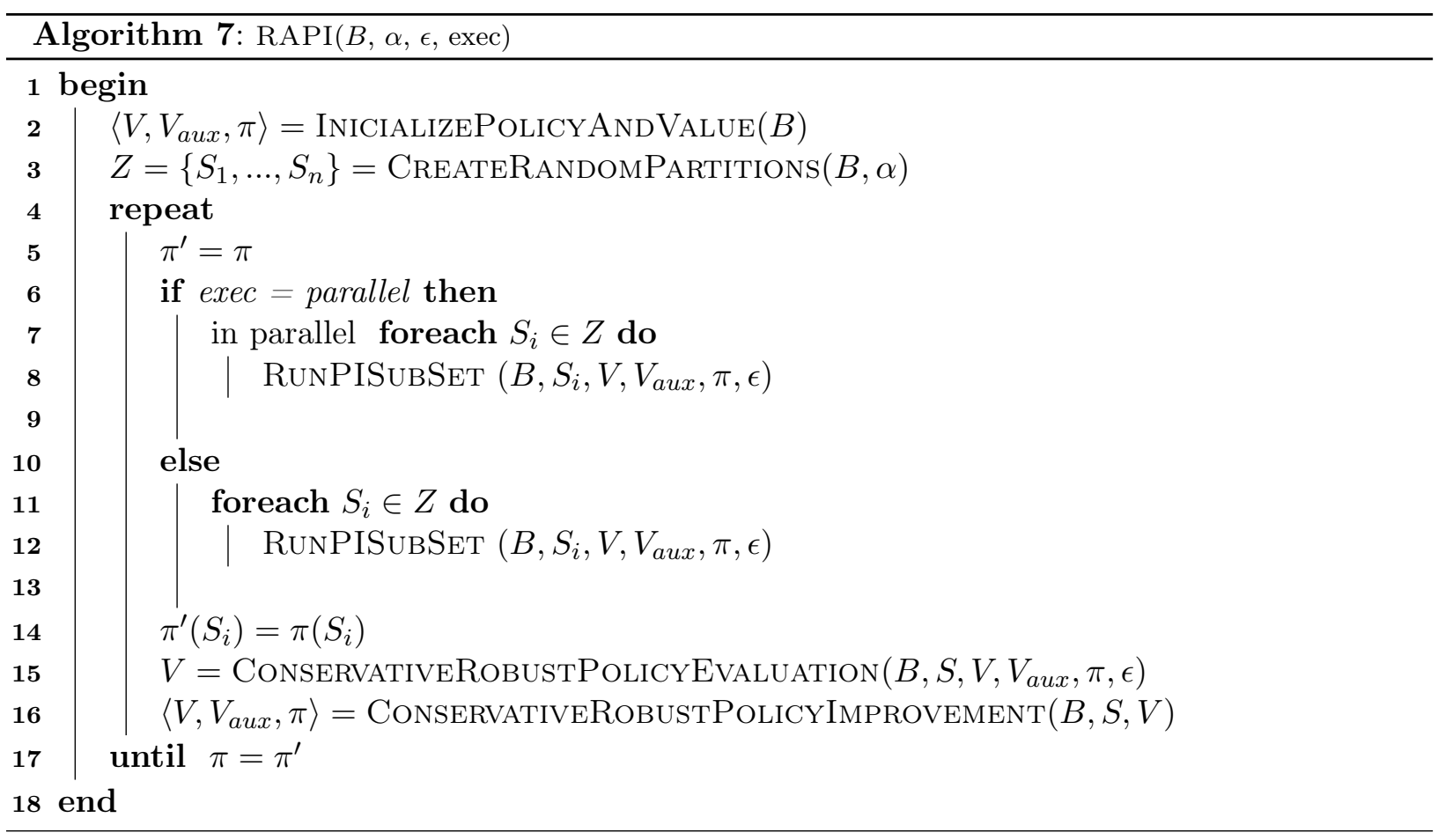

$\left(L\left(L^{k-1} V\right)\right)(s)$.

Prova: A Proposição 2 é provada como uma especialização da proposição correspondente para MDP-IPs [Ber77, BS78, Ber82, Ber07] uma vez que BMDPs são uma classe de MDP-IPs [GLD00].

Teorema 4. Convergência do algoritmo de Iteração de Política Assíncrono Robusto. Dado um BMDP e a Proposição 2, o algoritmo de Iteração de Política Assíncrono Robusto que utiliza avaliação de política conservativa robusta e melhoria de política conservativa robusta converge sob as seguintes condições:

i A função valor inicial $V_{1}^{0}, \ldots, V_{n}^{0}$ satisfaz $\underline{V} \leq V_{i}^{0} \leq \bar{V}$;

ii O número de atualizações de política é infinito; e

iii A troca de informação eventualmente ocorrerá.

Prova: Dada a proposição 2, o Teorema 4 é provado como uma especialização do resultado de Bertsekas e Yu [BY10a] para MDP-IPs, também sob as condições (i), (ii) e (iii), uma vez que BMDPs são uma subclasse dos MDP-IPs [GLD00].

Similar aos MDPs de horizonte infinito, a primeira condição é trivialmente satisfeita utilizando um número $\beta$ suficientemente grande (um limite superior da recompensa descontada acumulada esperada) tal que $\underline{V}=-\beta$ e $\bar{V}=\beta$, e.g., $\beta=R_{\max } /(1-\gamma)$, em que $R_{\max }=\max _{s, a}\{|R(s, a)|\}$ (ambos algoritmos, RAPI-parallel e RAPI-sequential, utilizam este limite superior).

A segunda condição (teórica): o número de atualizações é infinito, é implementado na prática com uma parada (Linha 16) checada depois de dois passos de atualização extra (Linhas 15 e 16) garantindo a convergência da política. Note que, ao aplicar estes dois passos de atualização síncronos extras para o conjunto completo de estados antes de verificar o critério de parada, a transmissão da informação atualizada de cada processador é forçada a ser realizada, similar ao algoritmo síncrono PI [Put94b].

Finalmente, a terceira condição é garantida para o RAPI-parallel se o sistema multiprocessador garante a troca de informação (i.e., ele funciona como esperado); e também pelo RAPI-sequential, uma vez que utiliza um único processador para executar meta-atualizações sequenciais. 


\subsection{Iteração de Política Topológico Robusto}

Inspirado no algoritmo de Iteração de Valor Topológico (TVI) [DMWG11], foi proposto um novo algoritmo para resolver BMDPs, Iteração de Política Topológica Robusta (Robust Topological Policy Iteration - RTPI) [RdBD19].

RTPI gera um Grafo Acíclico Dirigido (DAG) a partir do espaço de estados do BMDP, de modo que cada nó do DAG é um Componente Fortemente Conexo (SCC) $S_{i} \subseteq S$. Cada SCC é considerado um meta-estado, e a atualização sobre um meta-estado é chamada de meta-atualização. Em uma meta-atualização todos estados de um SCC são atualizados até a convergência, o que é equivalente a executar o algoritmo RunPISubSeT sobre $S_{i}$. Assim, RTPI executa uma meta-atualização em cada SCC em ordem topológica reversa, em que cada SCC precisa apenas de informações de SCCs que já convergiram, o que evita atualizações desnecessárias.

\subsubsection{Componentes fortemente conexos de um BMDP}

Em um BMDP, dados dois estados $s_{1}$ e $s_{2}$, e uma ação $a$, tal que $P_{\uparrow}\left(s_{2} \mid s_{1}, a\right)=[l, u]$, se pode ser escolhido $P\left(s_{2} \mid s_{1}, a\right) \in[l, u]$ maior que 0 , é dito que o estado $s_{2}$ é um sucessor de $s_{1}$, i.e., $s_{1}$ é dependente causal de $s_{2}$. Além disso, a relação de dependência causal é transitiva. Os estados $s_{1} \mathrm{e}$ $s_{2}$ são dependentes mutualmente causais se $s_{1}$ e $s_{2}$ são dependentes causais um do outro.

A construção de um SCC $S_{i} \subseteq S$ é feita pelo agrupamento de todos os estados $s \in S$, e suas respectivas arestas, que são dependentes mutualmente causais. Finalmente, o DAG completo de SCCs, chamado $G_{S C C}$, em que cada nó é um SCC, é obtido pela adição de arestas entre eles. Sejam $S_{1}$ e $S_{2}$ SCCs distintos, se há dois estados $s_{1} \in S_{1}$ e $s_{2} \in S_{2}$, e $s_{1}$ é causalmente dependente de $s_{2}$, então há uma aresta conectando os SCCs $S_{1}$ e $S_{2}$.

\subsubsection{Ordem de atualização ótima}

Dado um BMDP, se $s_{1}$ é causalmente dependente de $s_{2}$, e $s_{2}$ não é causalmente dependente de $s_{1}$, então a ordem de atualização ótima é $s_{2}$ e depois $s_{1}$. Note que, quando os estados são dependentes mutualmente causais a ordem de atualização ótima não é definida.

Teorema 5. Ordem de atualização ótima (caso acíclico): Dado um BMDP acíclico, então há uma ordem de atualização ótima, que quando seguida, a função valor (e política) ótima pode ser calculada executando apenas uma atualização por estado.

O Teorema 5 para BDMPs acíclicos é trivialmente provado seguindo o teorema de atualização ótima para MDPs [BY10b]. No caso de BMDP cíclico, o seguinte teorema pode ser especificado.

Teorema 6. Ordem de meta-atualização ótima (caso cíclico): Dado um BMDP cíclico, e seu DAG correspondente, em que cada nó é um SCC (meta-estado), então há uma ordem de meta-atualização ótima, que quando seguida, a função valor (e política) ótima pode ser calculada executando apenas uma meta-atualização por meta-estado.

O Teorema 6 também pode ser trivialmente provado seguindo o Teorema 5. Ambos, Teorema 5 e 6 podem ser aplicados com algoritmos baseados em Iteração de Valor ou Iteração de Política.

A Figura 5.2b mostra uma representação de um BMDP particionado em SCCs. Cada retângulo arredondado representa um SCC, e o número no canto superior direito representa sua ordem topológica. No exemplo, a ordem de atualização ótima dos meta-estados é a ordem topológica reversa, i.e. $3,2,1$ e 0 .

\subsubsection{Algoritmo RTPI}

O pseudo-código do algoritmo RTPI é mostrado no Algoritmo 8. Na Linha 2, a política e a função valor podem ser inicializadas com qualquer dos métodos heurísticos descritos na subseção 5.2 , i.e. IN0, IN1 e IN2. 


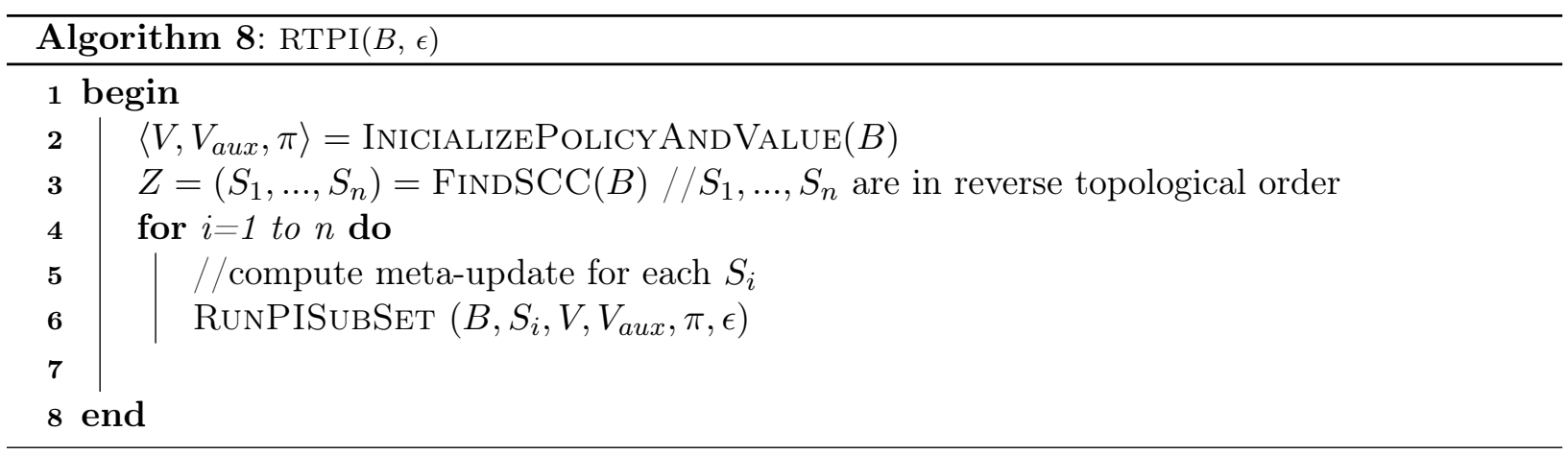

Na Linha 3, o conjunto de estados é particionado em SCCs, usando o algoritmo de Kosaraju [CLRS09], que cria um DAG $G_{S C C}$ de meta-estados e retorna uma lista $Z=\left(S_{1}, \ldots, S_{n}\right)$ de SCCs ordenados em ordem topológica reversa. Então é executada uma meta-atualização em cada $S_{i}$ seguindo esta ordem, o que é feito pela aplicação do algoritmo RunPISubSet na Linha 6. Note que RTPI não precisa usar operadores de atualização conservativos uma vez que seu assincronismo independe de atualizações em ordens arbitrárias e não realiza operações em paralelo.

Note que quando o grafo $G_{S C C}$, criado a partir do BMDP, tem apenas um SCC, i.e., quando todos estados são dependentes mutualmente causais, RTPI se comporta como o algoritmo RPI, com uma sobrecarga no tempo de execução para encontrar os SCCs. No entanto, essa sobrecarga é desprezível em relação ao tempo para resolver o BMDP. Assim, o desempenho do algoritmo RTPI depende principalmente da quantidade de componentes fortemente conexos no grafo $G_{S C C}$.

Teorema 7. Convergência do algoritmo de Iteração de Política Topológico Robusto.: Dado um BMDP, o algoritmo RTPI converge.

O teorema 7 é trivialmente provado seguindo o Teorema 5.

Neste capítulo foram apresentados (i) os algoritmos assíncronos de Iteração de Política baseados no particionamento do conjunto de estados, chamados RAPI e RTPI; e (ii) métodos de inicialização da função valor e política iniciais para BMDPs. A avaliação empírica desses algoritmos e métodos de inicialização é apresentada no Capítulo 6. 


\section{Capítulo 6}

\section{Resultados Experimentais}

Neste capítulo são apresentadas as contribuições práticas deste trabalho por meio da análise empírica dos algoritmos propostos: RAPI-sequential, RAPI-parallel e RTPI. O algoritmo RPI é usado como algoritmo base para as análises. Os algoritmos foram rodados em versões modificadas dos domínios de planejamento Layered MDP [DMWG11], Grid World [HZ01] e Race Track [BBS95], cujas probabilidades de transição foram definidas por intervalos. Adicionalmente, foi proposto um novo domínio chamado Medical [RdBD19]. Os algoritmos foram implementados em Java e os experimentos foram realizamos em uma máquina n1-standard da Google Cloud Platform com 8 núcleos e 30GB de memória.

Os experimentos realizados foram divididos em duas partes. Na primeira (Seção 6.2) foram testadas as versões sequencial e paralela do algoritmo RAPI para diversos tamanhos de subconjuntos. Na segunda (Seção 6.3) foram testados ambos os algoritmos RAPI e RTPI com inicialização IN0 e RTPI com inicialização IN2. Em ambos os casos, os resultados foram comparados com os resultados do algoritmo RPI nos quatro domínios introduzidos na Seção 6.1.

Em ambos os experimentos, o tempo total de execução dos algoritmos inclui os tempos de leitura da instância, inicialização da política e função valor iniciais (com as inicializações IN0, IN1 ou IN2), particionamento do conjunto de estados (aleatoriamente ou encontrando SCCs) e execução do algoritmo propriamente dito até a convergência do mesmo. A convergência do algoritmo representa que a política ótima foi encontrada.

Na seção 6.1 são apresentados os domínios de planejamento sob os quais os experimentos são executados. Na seção 6.2 o algoritmo RAPI é analisado levando em consideração o tipo de implementação (sequencial ou paralela), o número e o tamanho das partições para diferentes domínios e tamanhos de domínio. Na seção 6.3 os algoritmos RPI, a melhor versão do RAPI para cada instância e o algoritmo RTPI são comparados em cada uma das instâncias.

\subsection{Domínios de planejamento}

Os domínios de planejamento em que os algoritmos foram testados incluem domínios cujos estados podem depender mutuamente de todos os demais estados, isto é, que possuem apenas um SCC, até domínios que possuem vários SCCs. Os domínios e instâncias podem ser classificados como estruturados ou não estruturados de acordo com a quantidade de componentes fortemente conexos que possuem.

Domínio Layered. No Domínio Layered [DMWG11], um domínio de planejamento artificial, o conjunto de estados é dividido em $n_{l}$ camadas identificadas por inteiros de 1 a $n_{l}$. Estados em uma camada $j$ podem ter sucessores apenas em camadas maiores ou iguais a $j$. O parâmetro $n_{a}$ é o número máximo de ações que pode ser aplicada em cada estado. O número de sucessores de cada estado $s$ é um inteiro $1 \leq k \leq n_{s}{ }^{1}$. O intervalo inferior das probabilidades de transição foi definido

\footnotetext{
${ }^{1}$ Nos experimentos, fixamos $n_{a}=10$ e $n_{s}=10$.
} 
por $l=\min \{r, 1 / k\}$, e o limite superior foi definido por $u=\max \{r, 1 / k\}$, em que $r$ é um número aleatório e $0 \leq r \leq 1$. Este procedimento garante que a soma dos intervalos inferiores será menor ou igual a 1 e a soma dos intervalos superiores será maior ou igual a 1 para todo par estado-ação. O domínio Layered contém pelo menos $n_{l}-1$ componentes conexos. Uma vez que as transições entre as camadas são limitadas às mesmas ou camadas de maiores índices, estados de cada SCC deve pertencer à mesma camada. Na Figura 6.1, é mostrada uma instância do domínio Layered com 2 camadas e um total de 4 SCCs, em que as conexões entre as camadas ocorrem apenas da camada 0 para a camada 1.

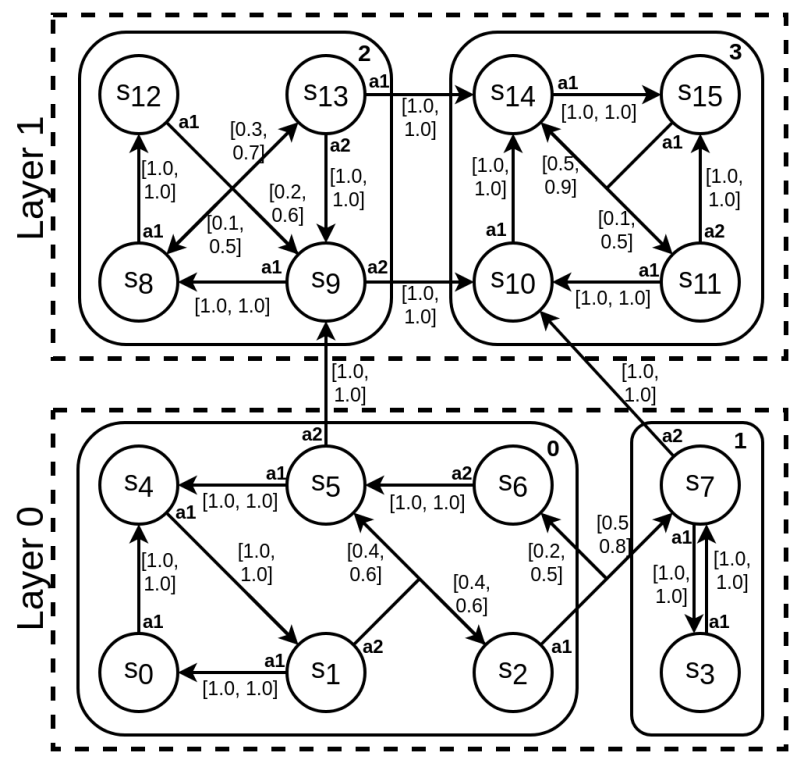

Figura 6.1: Exemplo de um problema BMDP no dominio Layered com 2 camadas e 4 SCCs.

Domínio Grid World. No domínio Grid World [HZ01], um robô se movimenta em um grid com $n$ linhas por $m$ colunas. O robô pode andar uma célula por vez nas direções cardeais norte, sul, leste ou oeste. O intervalo de probabilidades para que o robô termine na posição desejada é $[0.75$, 0.95] e termine em uma posição perpendicular à posição desejada é [0.05, 0.25]. Se o robô acerta uma parede, ele irá permanecer na mesma posição. A taxa de paredes é definida como $10 \%$ do tamanho do grid. Há uma recompensa de 1 para ações aplicadas no estado em que o robô está na posição superior direita (localização da meta) e 0 caso contrário. A figura 6.2 mostra um exemplo de uma instância do domínio Grid World com 10 linhas e 10 colunas, que possui 90 estados (quadrados brancos) e 10 paredes (quadrados cinzas escuros). A localização meta é representada em cinza claro.

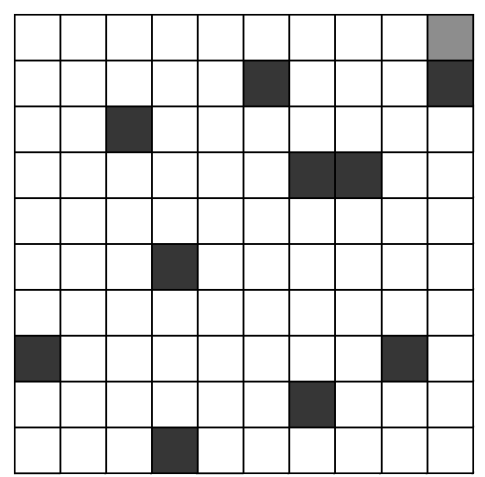

Figura 6.2: Exemplo de uma instância do dominio Grid World com 90 estados. 
Domínio Race Track. No domínio Race Track [BBS95], há um carro de corrida em uma pista representada por um grid com $n$ linhas e $m$ colunas, incluindo paredes dos dois lados de uma estrada com formato retangular. O problema original foi adaptado para um BMDP de horizonte infinito sem estado inicial (o carro pode inicializar em qualquer posição da pista) e há uma linha de chegada. Os estados são representados pela localização do carro no grid $(x, y)$ e sua velocidade nos eixos horizontal e vertical $\vec{v}=\left\langle v^{x}, v^{y}\right\rangle$. Em cada direção o carro pode desacelerar, manter a mesma velocidade ou acelerar, o que irá adicionar ao vetor $\vec{v}$ o vetor velocidade $\vec{u}=\left\langle u^{x}\right.$, $\left.u^{y}\right\rangle$, em que $u^{x}, u^{y} \in\{-1,0,1\}$. Sempre que o carro acertar uma parede, ele voltará para a última posição no grid com $\vec{v}=\langle 0,0\rangle$. A recompensa foi definida como 1 para estados na linha de chegada e 0 caso contrário. A figura 6.3 mostra um exemplo de uma instância do domínio Race Track, em que as 84 posições válidas no grid são representadas por quadrados brancos e as paredes são representadas por quadrados cinzas. Esse exemplo tem 1239 estados considerando as posições válidas e as velocidades possíveis em cada posição. Os quadrados cinzas claros indicam as posições da linha de chegada. O carro se movimenta a partir de qualquer posição em sentido horário a fim de alcançar a linha de chegada no menor tempo possível.

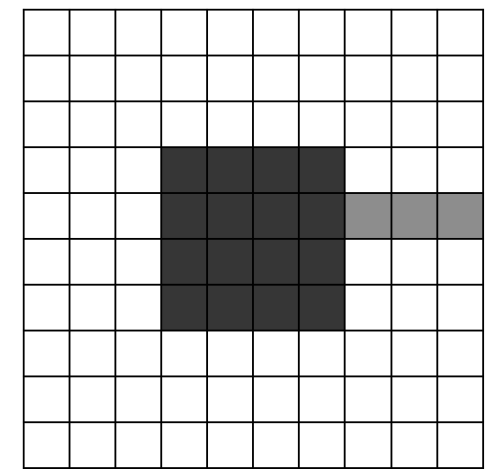

Figura 6.3: Exemplo de uma instância do dominio Race Track com 1239 estados.

Domínio Tratamento Médico. Neste domínio um paciente pode ser tratado para uma ou mais doenças diferentes, uma vez que uma doença pode ocorrer simultaneamente ou como consequência de outra (por exemplo, hipertensão, aterosclerose, acidente vascular cerebral, cardiopatia grave, cardiopatia irrecuperável, etc). O uso de intervalos de probabilidade pode capturar a dificuldade de definir probabilidade de obter sucesso em diferentes tratamentos considerando a idade do paciente, pertencimento a grupos de risco, entre outros. Este domínio é baseado em trabalhos anteriores sobre modelagem da evolução e tratamento de doenças específicas como MDP [SBSR04, TCdB07b, TT13]. A doença do paciente e sua condição de saúde, (por exemplo, testes para colesterol e nível de pressão sanguínea) são representados como variável de estados. As ações neste domínio são os possíveis tratamentos médicos, como tomar remédios, cirurgia, angioplastia, transplante, fazer nada (noop), entre outros. As probabilidades de transição condicionais de cada tratamento para cada doença é representada por uma Rede Bayesiana Dinâmica e a transição entre estados é sua probabilidade conjunta. Este domínio é interessante para comparar as soluções propostas com outras que não consideram a estrutura do domínio, uma vez que ele contém muitos SCCs para diferentes doenças e condições do paciente.

\subsection{RAPI-sequential vs RAPI-parallel}

Nesta seção as versões sequencial e paralelas do algoritmo RAPI são analisadas com inicialização IN0 como proposto em [RDdB16] para responder as questões: (1) RAPI-parallel é mais eficiente do que RAPI-sequential? (2) Qual é o número de subconjuntos ou número de estados por subconjunto do espaço de estados a fim de obter o melhor tempo de convergência para o algoritmo RAPI? 

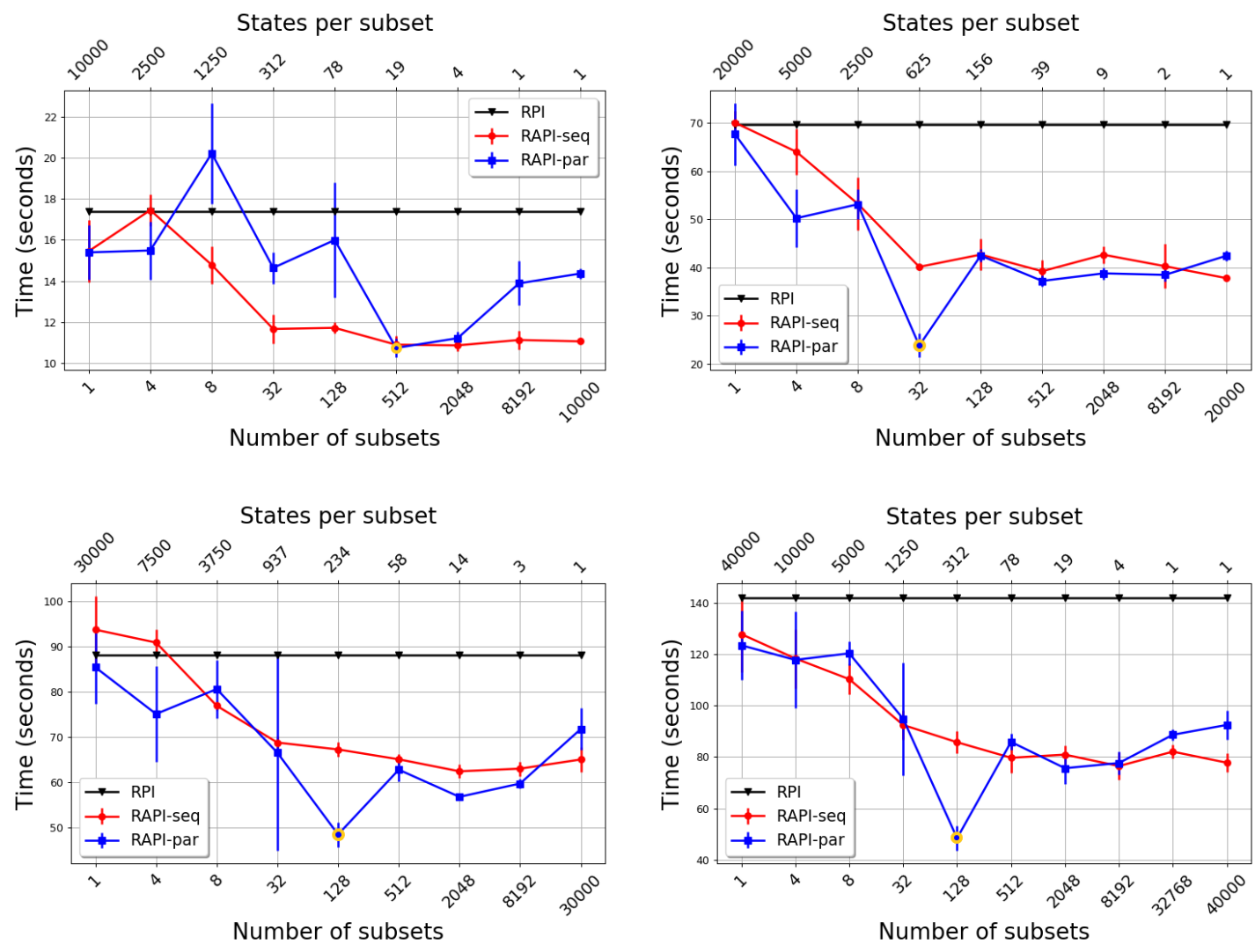

Figura 6.4: Tempo de convergência para 4 instâncias do dominio Layered com 10000, 20000, 30000 e 40000 estados, variando o número de subconjuntos. Linhas verticais indicam o desvio padrão.

As figuras 6.4, 6.5, 6.6 e 6.7 mostram o tempo de convergência dos algoritmos RAPI-sequential, RAPI-parallel e RPI ao resolver quatro instâncias dos domínos Layered, Grid World, Race Track e três instâncias do domínio Tratamento Médico, respectivamente, variando o número de subconjuntos, i.e., o tamanho da partição $|Z|$. Devido a escolha aleatória dos subconjuntos, são mostrados o tempo de convergência médio e o desvio padrão (linhas verticais nas figuras) para 5 execuções de cada experimento para os algoritmos RAPI-sequential e RAPI-parallel. Para o algoritmo RPI é mostrado apenas o tempo de uma execução pois não tem nenhum elemento aleatório.

Quando $|Z|=1$, o tempo de convergência de todos os algoritmos são aproximadamente o mesmo em todos os domínios, uma vez que o algoritmo RAPI é similar ao algoritmo RPI, mas com as atualizações conservativas ao invés de atualizações comuns. Considerando a variação do número de subconjuntos, ambas as versões do algoritmo RAPI tem diferente desempenho. Essa variação é dependente da versão do algoritmo, do domínio e do tamanho da instância. Em geral, ao aumentar o número de subconjuntos de $|Z|=1$ até $|Z|=|S|$ o tempo de convergência dos algoritmos diminui até um valor mínimo e depois volta a aumentar.

O algoritmo RAPI-sequential apresentou melhor desempenho em geral com muitos subconjuntos de poucos estados, sendo que seu desempenho se estabilizou de um número de subconjuntos em diante e teve uma pequena piora quando o número de subconjuntos se aproximava de $|S|$ em apenas algumas das instâncias dos domínios Grid World e Medical Treatment.

A versão paralela do algoritmo RAPI, por sua vez, apresentou uma grande piora no desempenho ao aumentar o número de subconjuntos, uma vez tendo atingido o melhor desempenho. Em alguns casos RAPI-parallel apresenou resultados piores do que o RPI. Isto se deve ao excesso de comunicação entre os subconjuntos que é um um comportamento comum em computação paralela: eventualmente, há uma sobrecarga de comunicação que implica em um custo computacional que domina o tempo de convergência gasto no processamento paralelo.

Apesar disso, para a maioria das instâncias, RAPI-parallel mostra um tempo de convergência 

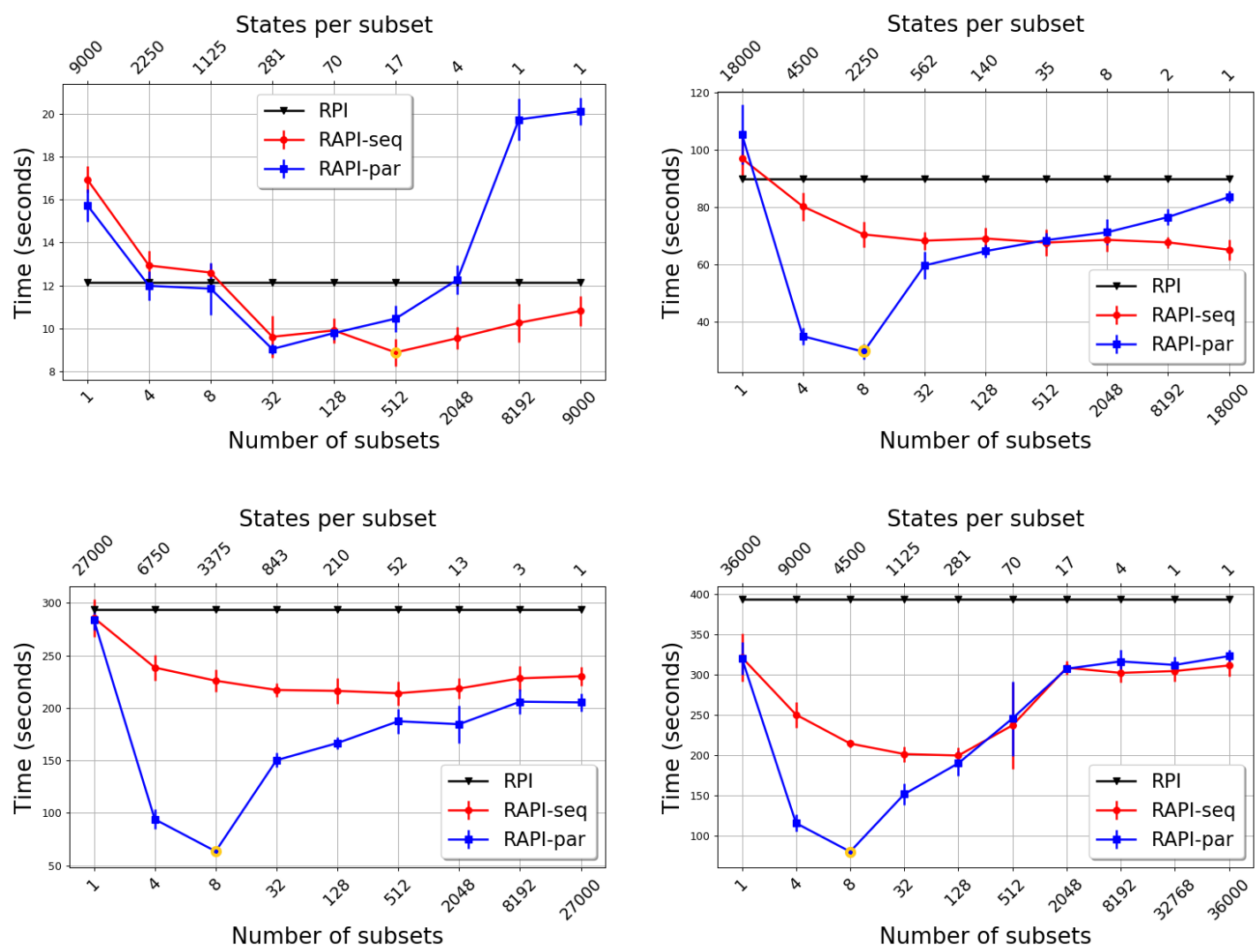

Figura 6.5: Tempo de convergência para 4 instâncias do domínio Grid World com 9000, 18000, 27000 e 36000 estados, variando o número de subconjuntos. Linhas verticais indicam o desvio padrão.

melhor ou igual ao RAPI-sequential (28 dentre 37 para o domínio Layered, 29 dentre 37 para o domínio Grid World, 15 dentre 33 para o domínio Race Track e 19 dentre 21 para o domínio Tratamento Médico).

É importante notar que a melhor execução do algoritmo RAPI em termos de tempo de execução foi obtida pela versão paralela do algoritmo RAPI. No entanto, mesmo com 8 processadores, os melhores casos obtiveram um desempenho apenas 2 a 3 vezes superior ao desempenho da versão sequencial, o que sugere que utilizar paralelismo pode não ser adequado para o caso de problemas modelados com BMDPs de horizonte infinito.

\subsection{Comparando RPI, RAPI* e RTPI}

Nesta seção são comparados os tempos de convergência dos algoritmos RPI, RAPI (parallel ou sequential) e RTPI, ambos inicializados com IN0. Os resultados do algoritmo RAPI consideram a versão do algoritmo e o tamanho da partição que resultou no menor tempo de convergência na seção 6.2 e é denotado por RAPI*. Além destes, também são mostrados os resultados do algoritmo RTPI inicializado com IN2, rotulado como RTPI-IN2.

Para o domínio Layered foram realizados dois experimentos: um variando o número de camadas e outro variando o número de estados. No primeiro experimento com o domínio Layered, o número de estados foi fixado em 50,000 e o número de camadas $n_{l}$ foi variado de 1 a $10 \mathrm{com}$ passos de 1 ; 10 a 100 com passos de 10 ; e 100 a 1,000 com passos de 100 .

Em todas as instâncias, o tempo de convergência do algoritmo RPI foi o maior entre os demais algoritmos, com exceção da instância com uma camada, em que o algoritmo RTPI ficou pior que o algoritmo RPI.

Na Figura 6.8a, para ambos algoritmos RPI e RAPI*, o tempo de convergência para diferentes 

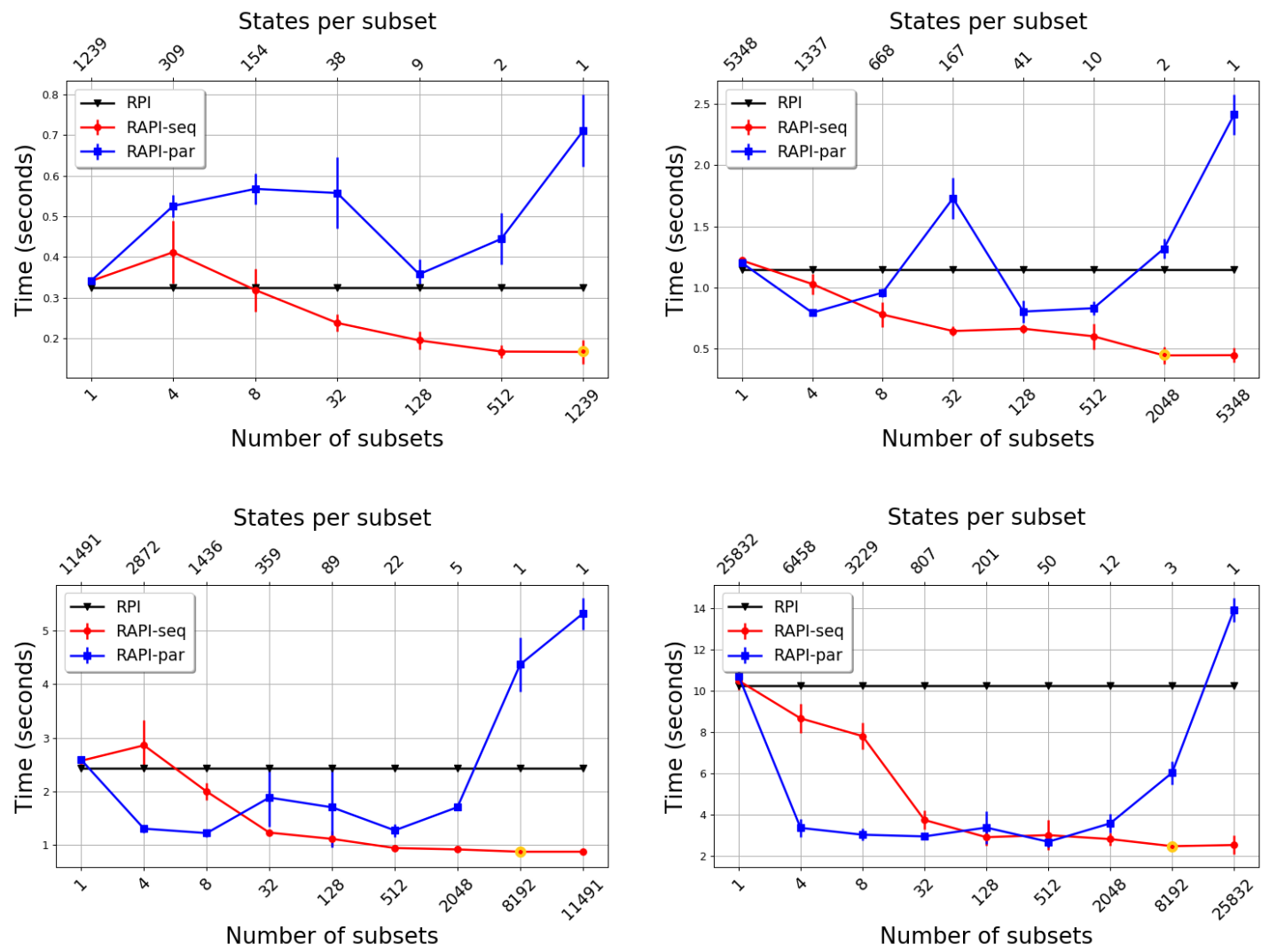

Figura 6.6: Tempo de convergência para 4 instâncias do dominio Race Track com 1239, 5348, 11491 e 25832 estados, variando o número de subconjuntos. Linhas verticais indicam o desvio padrão.

números de camadas parece ser independente do número de camadas. Entretanto, para RTPI e RTPI-IN2, enquanto o número de camadas aumenta o tempo de convergência diminui. Isso ocorre porque instâncias com mais camadas são mais estruturadas e portanto eles são mais simples de resolver com RTPI e RTPI-IN2. Considerando o melhor caso, RTPI e RTPI-IN2 foram até 32 e 33 vezes mais rápidos do que RPI, respectivamente.

RTPI-IN2 obteve desempenho melhor que RAPI* em todas as instâncias. RTPI por sua vez, obteve desempenho melhor que RAPI* apenas em instâncias com 3 camadas ou mais. Considerando os algoritmos topológicos, RTPI obteve desempenho equivalente ao RTPI-IN2 com instâncias com mais de 20 camadas. Esses resultados sugerem que inicializar um BMDP com a solução de um MDP tem uma boa influência no desempenho do algoritmo.

A Figura 6.8b mostra o resultado de experimentos no domínio Layered para instâncias com um número fixo de camadas, $n_{l}=10$, e variando o número de estados de 10,000 a 100,000 com passos de 10,000. Os resultados mostram que RTPI e RTPI-IN2 são até 12 e 23 times mais rápidos que RPI, respectivamente.

Na Figura 6.8c é mostrado o tempo de convergência para o domínio Grid World, com tamanhos de grid de 100x100, 100x200, ..., 100x1000 células. Uma vez que neste domínio há apenas um SCC, era esperado que o tempo de convergência do RTPI fosse equivalente ao tempo de RPI. No entanto, os experimentos sugerem que o uso de operadores conservativos podem ter influenciado um menor tempo de execução em relação ao algoritmo RPI.

Os algoritmos RAPI* e RTPI-IN2 tiveram tempo de execução similar. No entanto, RAPI* requer o conhecimento do melhor tamanho de partição para cada instância, o que mais uma vez sugere que mais importante do que ter poder computacional, é o uso de maneiras adequadas para diminuir o número de cálculos que são dispendiosos.

A Figura 6.8d mostra o tempo de execução do domínio Race Track. Foram geradas 9 instância com 10x10, 15x15, ..., 50x50 posições. Este é um domínio com poucos SCCs e a maioria dos estados 

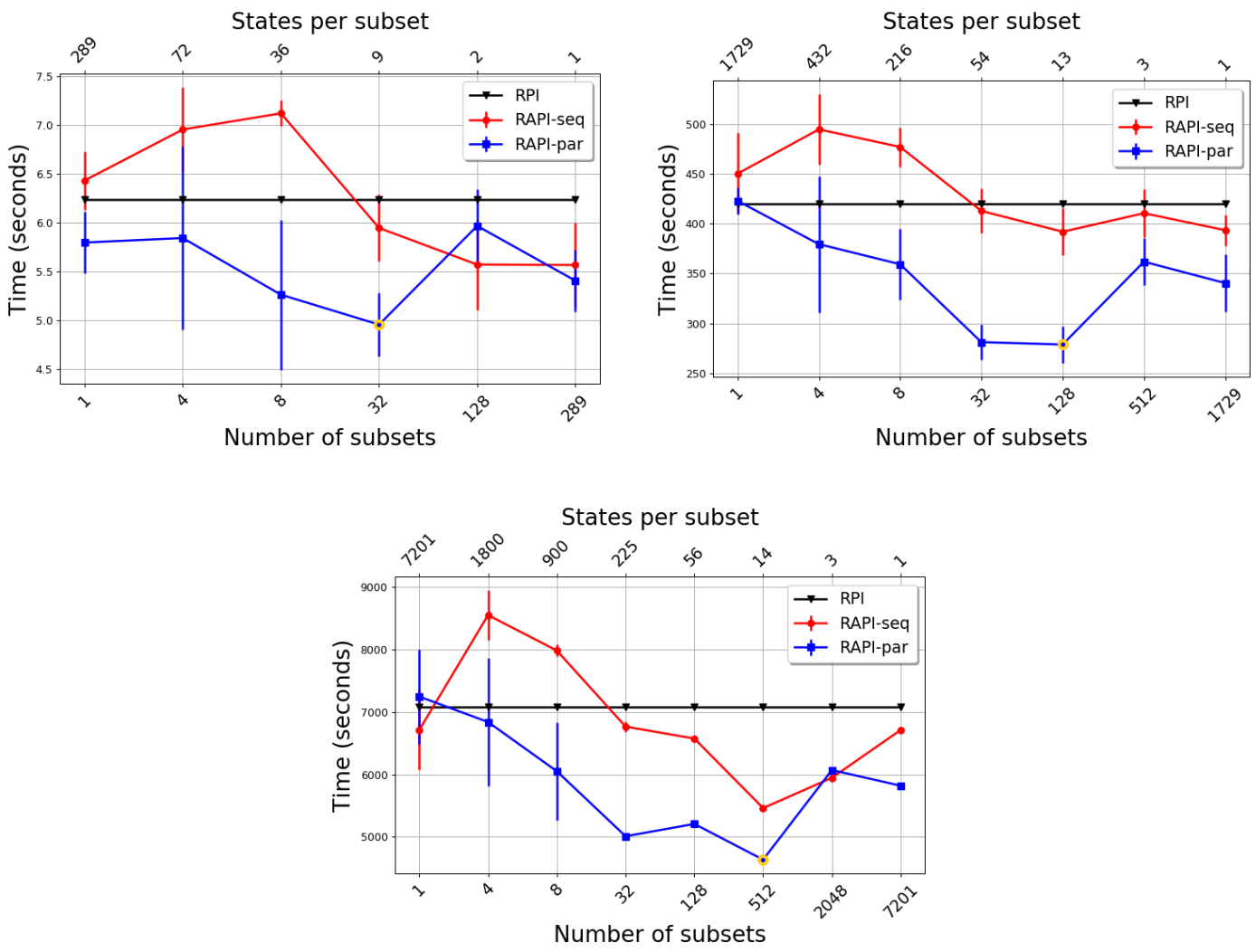

Figura 6.7: Tempo de convergência para 3 instâncias do domínio Tratamento Médico com 289, 1729 e 7201 estados, variando o número de subconjuntos. Linhas verticais indicam o desvio padrão.

pertencem a um grande SCC. Neste domínio RTPI e RTPI-IN2 são até 4 e 8 vezes mais rápidos do que RPI, respectivamente. Note que uma vez que este domínio tem um pouco mais de estrutura, o tempo de convergência de RTPI-IN2 é um pouco melhor do que RAPI*.

Figura 6.8e mostra o tempo de convergência para o domínio Tratamento Médico, com 289, 1729 e 7201 estados. Os experimentos mostram que RTPI e RTPI-IN2 são até 15 e 31 vezes mais rápidos do que RPI, respectivamente.

Concluindo, pode-se notar que quanto mais estruturado é o domínio, melhor é o desempenho do algoritmo RTPI, com qualquer que seja a sua inicialização. Nos domínios mais estruturados Layered e Tratamento Médico, RTPI-IN2 obteve os melhores tempos de convergências (até 33 vezes mais rápido no domínio Layered e até 31 vezes mais rápido no domínio Tratamento Médico quando comparado ao tempo de convergência do algoritmo do RPI). Em problemas com pouca (ou nenhuma) estrutura, como Grid World e Race Track, os melhores resultados foram obtidos com a versão de RTPI com uma inicialização IN2, o que foi tão vantajoso quanto utilizar 8 vezes mais poder computacional através de paralelismo e com uma melhoria de 2 a 3 vezes quando comparado ao desempenho do algoritmo RPI. 


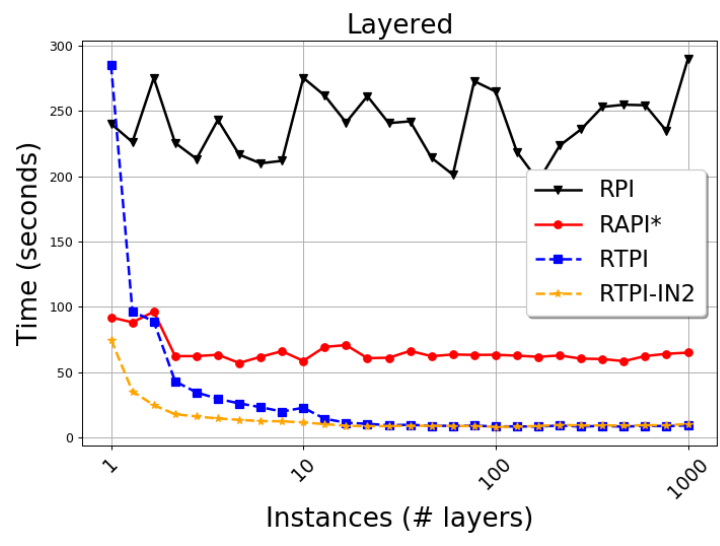

(a)

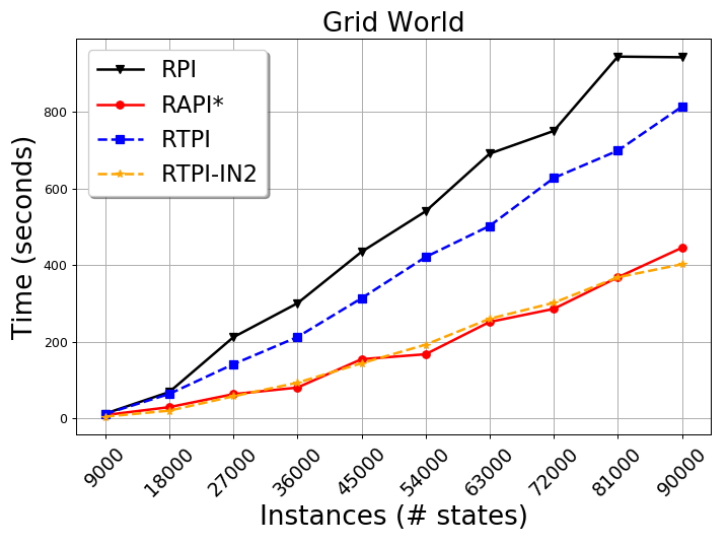

(c)

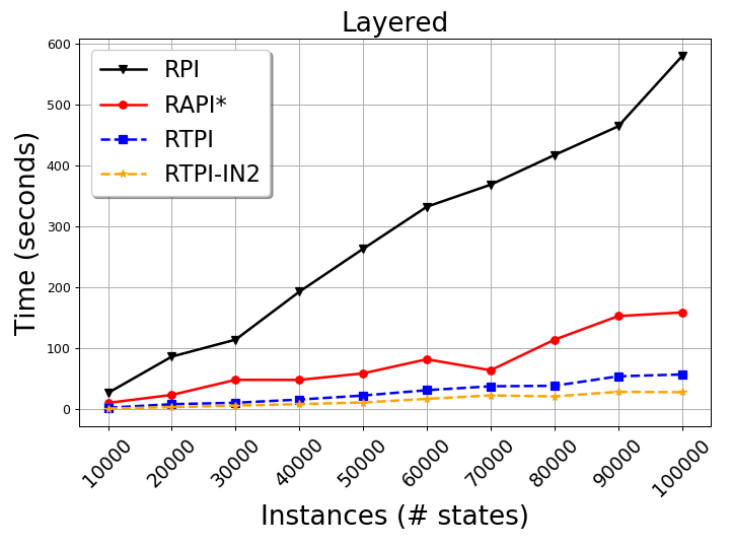

(b)

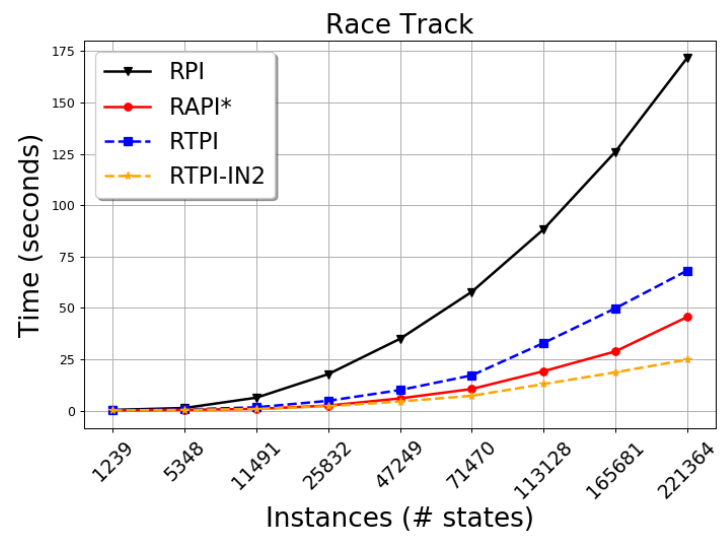

(d)

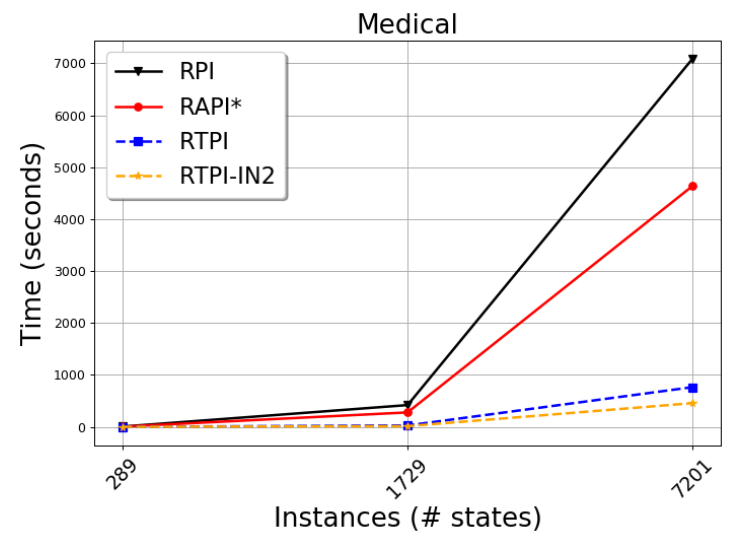

(e)

Figura 6.8: Tempo de convergência de RPI, RAPI* e RTPI em 4 dominios. (a) Dominio Layered variando o número de camadas; (b) Dominio Layered variando o número de estado e $n_{l}=10$; (c) Dominio Grid World variando o número de estados; (d) Domínio Race Track variando o número de estados; e (e) Dominio Médico variando o número de estados. 


\section{Capítulo 7}

\section{Trabalhos relacionados}

Neste capítulo serão apresentados alguns trabalhos relacionados com BMDPs de horizonte infinito, entre eles: BMDP de horizonte indeterminado e horizonte finito (Seção 7.1), MDPs com Transição Valorada por Conjuntos (MDPST) (Seção 7.2), Cadeias de Markov Imprecisas, MDPs Parcialmente Observáveis com Probabilidades Intervalares (BPOMDPs), Processos de Decisão Semi Markovianos com Probabilidades Intervalares (BSMDP) e MDPs Possibilísticos (Seção 7.3).

\subsection{Outros horizontes para BMDPs}

Um MDP de horizonte indeterminado é um MDP que além da tupla $M=\langle S, A, P, R, \gamma\rangle$ também inclui uma distribuição de probabilidades para um estado inicial e um conjunto de estados metas, além de ser mais comum utilizar uma função de custos $C$ ao invés da função de recompensa $R$. Tal MDP também é conhecido como problema do caminho estocástico mais curto (Stochastic Shortest Path - SSP) [MK12b].

A solução de um SSP é uma política ótima que tem como objetivo levar o agente até a meta. Geralmente, o critério utilizado para resolver um SSP é o de minimização do custo acumulado esperado de chegar até a meta, que é equivalente à maximização da recompensa acumulada descontada em um MDP de horizonte infinito, ao se tratar da equação de Bellman. Também é possível pensar no critério de maximização da probabilidade de alcançar a meta, chamado de MAXPROB [KMWG11]. Além destes, pode-se utilizar um critério dual, que considera primeiro a maximização da probabilidade de alcançar a meta, e então depois a minimização do custo.

Analogamente a um SSP, um BMDP de horizonte indeterminado (BSSP - Bounded-Parameter Stochastic-Shortest Path) tem como objetivo alcançar uma meta a partir do estado inicial com o custo esperado mínimo. Assim, a política ótima é uma política parcial sobre os estados alcançáveis a partir do estado inicial. Há algoritmos eficientes para resolver BSSP tais como Robust LAO*[CSYL06], LRTDP Robusto [BA05], B²RTDP Robusto [FDdB14] e ILAO* Robusto [MDNdB16].

Também é possível resolver BMDPs de horizonte finito. No entanto, a complexidade computacional para resolver tais problemas é NP-completo [Sch15], que é consideravelmente maior do que a complexidade P-completo para resolver BMDPs de horizonte infinito e indeterminado. O principal motivo é que, ao considerar cada tempo individualmente a partir do último horizonte pode levar a atribuição de probabilidades inconsistentes através dos diferentes estados, o que impossibilita o uso da abordagem de programação dinâmica. Além deste motivo, explorar as dependências entre os mesmos estados em diferentes tempos dificulta a resolução do problema [Sch15].

Em [TB07] é considerado um novo critério para BMDPs de horizonte infinito, que ao invés de considerar o critério de recompensa acumulada descontada, considera recompensa média a longo prazo através de políticas ótimas de Blackwell. 


\subsection{MDPST}

Um Processo de Decisão Markoviano com transição valorada por conjuntos (Markovian Decision Process with Set-valued Transition - MDPST) é uma subclasse dos MDP-IPs, em que as probabilidades de transição são definidas para conjuntos de estados [TCdB07a]. É esperado que o resultado da interação do agente com o ambiente produza qualquer um dos estados do conjunto de estados sucessores de maneira não determinística. Desta forma, a função de transição $m(k \mid s, a)$ atribui uma probabilidade de alcançar o conjunto de estados $k$ ao executar uma ação $a \mathrm{em}$ um estado $s$. $\mathrm{O}$ estado $s^{\prime} \in k$ resultante é escolhido de forma não determinística.

Os MDPSTs são mais gerais que os modelos probabilísticos e não determinísticos, pois eles consideram a semântica de cada um desses modelos, como também a combinação de ambos em qualquer proporção possível.

Trevizan et al (20017) mostram que as transições de um MDPST podem ser convertidas para transições de um MDP-IP, através da definição de um conjunto de restrições lineares a partir das transições do MDPST. A partir do princípio de otimalidade de Bellman para MDP-IPs (Equação 3.3), e considerando o critério maximin, é gerado o princípio de otimalidade de Bellman para MDPSTs [TCdB07a]:

$$
V^{*}(s)=\max _{a \in A}\left\{R(s, a)+\sum_{k} m(k \mid s, a) \min _{s^{\prime} \in k} V^{*}\left(s^{\prime}\right)\right\} .
$$

Note que o min, comparando com a Equação 3.3, foi empurrado para dentro da soma, o que significa que para resolver um MDPST é preciso fazer uma otimização local usando um algoritmo guloso, o que o torna esse cálculo mais eficiente que a versão para MDP-IPs.

Os algoritmos básicos para resolver MDPSTs de horizonte infinito são versões adaptadas dos algoritmos de iteração de política e iteração de valor para MDPs, que substituem o princípio de otimalidade de Bellman de MDPs para MDPSTs.

Um MDPST também pode ser definido de modo a representar o problema do caminho estocástico mínimo. O problema é chamado de problema do caminho estocástico mínimo com transição valorada por conjuntos (Stochastic Shortest Path with Set-valued Transition - SPST) [TCdB07a, Tre06]. O algoritmo RTPD foi adaptado para resolver SPSTs [Tre06].

Tanto BMDPs quanto MDPSTs podem ser representados por MDP-IPs e resolvidos por seus algoritmos. No entanto, estes modelos são mais limitados do que MDP-IPs, pois não são capazes de expressar restrições gerais. Além disso, BMDPs e MDPSTs não são equivalentes. Existem problemas modelados como MDPSTs que não podem ser modelados como BMDPs, e vice-versa [TCdB07a].

\subsection{Outros modelos com probabilidades imprecisas e incerteza}

Os Processos de Decisão Markovianos com Probabilidades Imprecisas, também conhecidos como MDPs Incertos, representam as funções de transição com parâmetros. Similar a [GLD00], alguns trabalhos assumem que os parâmetros são elementos de um conjunto delimitado conhecido e usam técnicas de otimização robusta para resolver estes problemas tais como [Iye05, NG05, MMX12, DSdB11b]. Nosso trabalho também considera um tipo específico de conjuntos delimitados dados por intervalos. Alternativamente, alguns trabalhos assumem que os parâmetros são variáveis aleatórias que seguem alguma distribuição tais como [DM10, XM12]. Nosso trabalho não considera uma distribuição de probabilidade sobre as probabilidades.

\subsubsection{Cadeias de Markov Imprecisas}

Cadeias de Markov são um tipo de processos estocásticos usados para modelar sistemas com dinâmica incertas ou imprecisas. Algumas extensões incluem Cadeias de Markov com probabilidades intervalares [CDdRCK03, DCHM94, KU02, Šku06], Cadeias-conjunto de Markov [Har98] e Cadeias de Markov com Probabilidades Imprecisas, também conhecidas como Cadeias de Markov Imprecisas 
[Wa191, dCHQ09, DCDBL16]. Estes tipos de processos lidam com probabilidades imprecisas e podem ser vistas como um Processo de Decisão Markoviano com Probabilidades Imprecisas com uma política fixa, i.e., em que um agente não tem que decidir qual ação executar em cada estado. Neste cenário, há trabalhos interessados em um estudo formal do comportamento limite de tais sistemas, com intervalos de probabilidades e conjuntos gerais de probabilidades [dCHQ09] [DCDBL16].

Algumas definições de Cadeias de Markov Imprecisas dadas em [dCHQ09] são diretamente relacionadas às definições dadas na Seção 5.4.1, tais como: (i) a relação de acessibilidade que corresponde à relação de dependência causal; (ii) a relação de comunicação que corresponde à dependência mutualmente causal; e (iii) a classe de comunicação que corresponde a um componente fortemente conexo (um meta-estado) de um BMDP.

\subsubsection{BPOMDPs e BSMDPs}

Em [NL08] os Processos de Decisão Markovianos Parcialmente Observáveis com Probabilidades Intervalares (Bounded-Parameter Partially Observable Markov Decision Processes - BPOMDP) são formulados. No mesmo trabalho são propostos e avaliados algoritmos baseados em iteração de valor. Em um trabalho posterior é apresentada uma abordagem otimista para calcular a função valor ótima de um BPOMDP, além de um algoritmo de iteração de política para resolver o modelo [NL13].

Em [BDS17], o modelo BMDP é estendido para Processos de Decisão Semi Markovianos com Probabilidades Intervalares (Bounded-Parameter Semi Markov Decision Processes - BSMDP) e os autores introduzem novas variações de iteração de valor e iteração de política para resolver BSMDPs. Neste trabalho, não lidamos com observabilidade parcial nem com processos semi Markovianos.

\subsubsection{MDPs Possibilísticos}

Os MDPs Possibilísticos [Sab01] consideram uma abordagem qualitativa para modelar as transições entre estados baseada em teoria da possibilidade para representação de incerteza. O modelo tenta representar a preferência sobre estados apenas com uma relação de ordenação entre eles. Assim, é proposta uma correspondência da equação de Bellman para MDPs possibilísticos [SFL98]. Os MDPs Possibilísticos podem ser resolvidos considerando horizontes finitos e infinitos, por meio de versões adaptadas dos algoritmos de Iteração de Valor e Iteração de Política para MDPs. 


\section{Capítulo 8}

\section{Considerações Finais}

Neste trabalho, o modelo BMDP foi revisitado no contexto de horizonte infinito, através da proposição de novos algoritmos assíncronos baseados em Iteração de Política e da avaliação empírica destes em domínios de planejamento existentes e em um novo domínio proposto.

Como primeiro resultado, foi proposto um algoritmo baseado no particionamento aleatório do conjunto de estados, o RAPI, que pode ser utilizado tanto sequencialmente como paralelamente. No entanto, ao longo do trabalho, que contou com uma análise empírica extensiva, foi mostrado que a utilização de computação paralela com particionamento aleatório pode não ser uma boa alternativa para resolver BMDPs, por não conseguir obter vantagens computacionais na proporção do poder computacional utilizado, além da dificuldade de definir os parâmetros que tornam a execução deste algoritmo mais eficiente. Assim, foi considerado o uso do particionamento do conjunto de estados a partir da estrutura do grafo do problema, o que resultou no algoritmo RTPI, que particiona o espaço de estados em componentes fortemente conexos. No entanto, o desempenho do algoritmo RTPI é dependente das características do problema, e em casos em que o grafo é cíclico em grande parte dos estados o seu desempenho é prejudicado. Como solução, foram propostas formas de inicializar a função valor e política iniciais de modo a diminuir o número de atualizações necessárias, e consequentemente, o tempo de execução, dos algoritmos para resolver BMDPs. A inicialização com melhores resultados, chamada de IN2, foi obtida pela resolução de um MDP criado a partir do BMDP.

Os experimentos deste trabalho foram divididos em duas partes. Na primeira parte, o objetivo dos experimentos era verificar o comportamento do algoritmo RAPI, em suas diferentes versões, para diferentes tamanhos de particionamento do conjunto de estados. Como resultado deste experimentos, foi verificado que o desempenho do algoritmo RAPI-parallel é melhor do que o do algoritmo RAPI-sequential nos domínios testados, mas não na proporção do poder computacional utilizado. Além disso, o desempenho do RAPI-parallel depende fortemente do tamanho das partições, do tamanho do problema e do número de processadores utilizados, o que não é trivial para definir. $\mathrm{O}$ mesmo é válido para o RAPI-sequential, mas em geral partições com poucos estados tendem a ter os melhores resultados para este algoritmo. Na segunda parte foram testados os algoritmos propostos, RAPI e RTPI com o algoritmo RPI. Foi utilizada a melhor execução do RAPI, obtida no primeiro experimento, chamada de RAPI*, o algoritmo RTPI com inicialização IN0 e IN2, respectivamente RTPI e RTPI-IN2. Quanto mais estruturado o problema, melhores foram os resultados do algoritmo RTPI. No entanto, quando o problema é pouco estruturado, o desempenho do algoritmo RTPI é similar ao algoritmo RPI, o que é superado pelo uso da inicialização IN2. Os experimentos realizados sugerem que o algoritmo RTPI-IN2 possui melhor desempenho em quaisquer dos domínios testados ou é pelo menos tão bom quanto a melhor execução do algoritmo RAPI*.

\subsection{Resumo das contribuições}

As principais contribuições deste trabalho foram apresentadas nas seguintes seções e capítulos: 
- No Capítulo 5 são propostos algoritmos assíncronos baseados em Iteração de Política para BMDPs que particionam o conjunto de estados aleatoriamente (RAPI) ou encontrando SCCs (RTPI). No algoritmo RAPI (Seção 5.3), o conjunto de estados $S$ é particionado aleatoriamente em subconjuntos de estados $S_{i}$, e cada subconjunto é atualizado sequencialmente com um único processador ou paralelamente em um sistema multiprocessador até a convergência parcial de cada subconjunto. No algoritmo RTPI (Seção 5.4), o conjunto $S$ é particionado em SCCs, que são atualizados em ordem topológica reversa. O algoritmo converge mediante a atualização de apenas uma meta-atualização para cada SCC. Foram apresentadas também as provas de convergência dos algoritmos propostos.

- Na Seção 5.2 são propostas heurísticas de inicialização para a função valor e políticas dos algoritmos propostos. A inicialização da função valor e da política para os algoritmos propostos tem impacto no tempo de convergência.

- No Capítulo 6 foi realizada a avaliação empírica dos algoritmos propostos em domínios de planejamento com tipos variados de estruturas. Os algoritmos também são avaliados em um novo domínio chamado de Tratamento Médico. Este domínio (descrito na Seção 6.1) apresenta potencial de utilização real e apresenta uma boa estrutura em termos de componentes fortemente conexos.

\subsection{Publicações geradas}

O algoritmo RAPI foi publicado no XIII Encontro Anual de Inteligência Artificial e Computacional (ENIAC 2016) no artigo Distributed and Asynchronous Policy Iteration for Bounded Parameter Markov Decision Processes [RDdB16], que recebeu menção honrosa na premiação de melhores trabalhos do Encontro.

O algoritmo RTPI foi publicado no periódico International Journal of Approximate Reasoning em 2019, no artigo intitulado Robust Topological Policy Iteration for Infinite Horizon Bounded Markov Decision Processes [RdBD19].

\subsection{Trabalhos futuros}

Entre as possíveis extensões deste trabalho estão:

- Utilização de particionamento do espaço de estados e atualização dessas partições com métodos de priorização de estados por exemplo utilizando uma versão modificada do algoritmo Prioritized Sweeping [MA93]. Essa abordagem pode eliminar redundância na atualização dos estados e melhorar a eficiência de algoritmos iterativos [WS05].

- Utilização de particionamento do espaço de estados com técnicas de deteç̧ão de comunidade em grafos [For10], isto pode ajudar a resolver cada partição mais eficientemente, mesmo que de forma síncrona, uma vez sua divisão em clusters sugere que os estados possam ter mais dependência entre si.

- Proposição do algoritmo de Iteração de Política Intervalar e de outras formas de ordenação de funções valores intervalares.

- Comparação dos algoritmos propostos com técnicas eficientes para MDP-IP fatorados, a fim de verificar se é mais vantajoso resolver tais problemas com esses algoritmos específicos para BMDPs ou resolver com algoritmos gerais para MDP-IPs fatorados. 


\section{Referências Bibliográficas}

[BA05] Olivier Buffet e Douglas Aberdeen. Robust planning with LRTDP. Em Proceedings of International Joint Conferences on Artificial Intelligence, páginas 1214-1219, 2005. 2,47

[BBS95] Andrew G. Barto, Steven J. Bradtke e Satinder P. Singh. Learning to act using real-time dynamic programming. Artificial Intelligence, 72(1):81-138, 1995. 39, 41

[BDS17] Peter Buchholz, Iryna Dohndorf e Dimitri Scheftelowitsch. Analysis of Markov decision processes under parameter uncertainty. Em Computer Performance Engineering, páginas 3-18, Cham, 2017. Springer International Publishing. 49

[Bel57] R. E. Bellman. Dynamic Programming. Princeton University Press, USA, 1957. 6

[Ber77] Dimitri P. Bertsekas. Monotone mappings with application in dynamic programming. SIAM Journal on Control and Optimization, 15(3):438-464, 1977. 9, 36

[Ber82] Dimitri P. Bertsekas. Distributed dynamic programming. IEEE transactions on Automatic Control, 27(3):610-616, 1982. 9, 36

[Ber01] Dimitri P Bertsekas. Dynamic programming and optimal control, volume 2. Athena Scientific Belmont, MA, 2000-2001. 10

[Ber07] Dimitri P. Bertsekas. Dynamic Programming and Optimal Control, Vol. II. Athena Scientific, 3rd edição, 2007. 9, 36

$\left[\mathrm{BFG}^{+}\right.$93] Ruth Iris Bahar, Erica A. Frohm, Charles M. Gaona, Gary D. Hachtel, Enrico Macii, Abelardo Pardo e Fabio Somenzi. Algebraic decision diagrams and their applications. Em Proceedings of the International Conference on Computer-Aided Design (ICCAD), páginas 188-191, Los Alamitos, CA, USA, 1993. IEEE Computer Society Press. 11

[BHD99] Craig Boutilier, Steve Hanks e Thomas Dean. Decision-theoretic planning: Structural assumptions and computational leverage. Journal of Artificial Intelligence Research, 11:1-94, 1999. 11

[BS78] Dimitri P. Bertsekas e Steven E. Shreve. Stochastic Optimal Control: The DiscreteTime Case. Academic Press, Inc., Orlando, FL, USA, 1978. 9, 36

[BY10a] Dimitri P. Bertekas e Huizhen Yu. Distributed asynchronous policy iteration in dynamic programming. Em 48th Annual Allerton Conference on Communication, Control, and Computing, páginas 1368-1375, 2010. 6, 7, 9, 34, 36

[BY10b] Dimitri P Bertsekas e Huizhen Yu. Distributed asynchronous policy iteration in dynamic programming. Em Communication, Control, and Computing (Allerton), 2010 48th Annual Allerton Conference on, páginas 1368-1375. IEEE, 2010. 1, 2, 9, 37 
[CDdRCK03] Marcilia Andrade Campos, Graçaliz Pereira Dimuro, Antônio Carlos da Rocha Costa e Vladik Kreinovich. Computing 2-step predictions for interval-valued finite stationary markov chains. Computing, 7:1-2004, 2003. 48

[CLRS09] Thomas H. Cormen, Charles E. Leiserson, Ronald L. Rivest e Clifford Stein. Introduction to Algorithms. The MIT Press, 3rd edição, 2009. 38

[Coz00] Fabio G. Cozman. Credal networks. Artificial Intelligence, 120:199-233, 2000. 17

[Coz05] Fabio G. Cozman. Graphical models for imprecise probabilities. International Journal of Approximate Reasoning, 39(2-3):167-184, 2005. 17

[CSYL06] Shulin Cui, Jigui Sun, Minghao Yin e Shuai Lu. Solving uncertain Markov decision problems: An interval-based method. Em Advances in Natural Computation, páginas 948-957, 2006. 2, 47

[DCDBL16] Gert De Cooman, Jasper De Bock e Stavros Lopatatzidis. Imprecise stochastic processes in discrete time: global models, imprecise markov chains, and ergodic theorems. International Journal of Approximate Reasoning, 76:18-46, 2016. 49

[DCHM94] Luis M De Campos, Juan F Huete e Serafín Moral. Probability intervals: a tool for uncertain reasoning. International Journal of Uncertainty, Fuzziness and KnowledgeBased Systems, 2(02):167-196, 1994. 48

[dCHQ09] Gert de Cooman, Filip Hermans e Erik Quaeghebeur. Imprecise Markov chains and their limit behavior. Probability in the Engineering and Informational Sciences, 23(4):597-635, 2009. 23, 49

[DdBCS09] Karina Valdivia Delgado, Leliane Nunes de Barros, Fabio G. Cozman e Ricardo Shirota. Representing and Solving Factored Markov Decision Processes with Imprecise Probabilities. Em Proceedings ISIPTA, Durham, United Kingdom, 2009. 18

[DdBCS11a] Karina V. Delgado, Leliane N. de Barros, Fabio G. Cozman e Scott Sanner. Using Mathematical Programming to Solve Factored Markov Decision Processes with Imprecise Probabilities. International Journal of Approximate Reasoning, 52(7):10001017, 2011. 18

[DdBCS11b] Karina Valdivia Delgado, Leliane Nunes de Barros, Fabio Gagliardi Cozman e Scott Sanner. Using mathematical programming to solve Factored Markov Decision Processes with Imprecise Probabilities. International Journal of Approximate Reasoning, 52(7):1000 - 1017, 2011. Selected Papers - Uncertain Reasoning Track - FLAIRS 2009. 26

[DdBDS16] Karina V Delgado, Leliane N de Barros, Daniel B Dias e Scott Sanner. Real-time dynamic programming for Markov Decision Processes with Imprecise Probabilities. Artificial Intelligence, 230:192-223, 2016. 1

[DG07] Peng Dai e Judy Goldsmith. Topological value iteration algorithm for Markov Decision Processes. Em Manuela M. Veloso, editor, Proceedings of International Joint Conferences on Artificial Intelligence, páginas 1860-1865, 2007. 6, 7

[DM10] Erick Delage e Shie Mannor. Percentile optimization for Markov decision processes with parameter uncertainty. Operations research, 58(1):203-213, 2010. 48

[DMWG11] Peng Dai, Mausam, Daniel S. Weld e Judy Goldsmith. Topological value iteration algorithms. Journal of Artificial Intelligence Research, 42:181-209, 2011. 10, 37, 39 
[DSdB11a] Karina Valdivia Delgado, Scott Sanner e Leliane Nunes de Barros. Efficient solutions to factored MDPs with imprecise transition Probabilities. Artificial Intelligence, 175(9-10):1498-1527, 2011. xv, 14, 17, 18

[DSdB11b] Karina Valdivia Delgado, Scott Sanner e Leliane Nunes de Barros. Efficient solutions to factored MDPs with imprecise transition Probabilities. Artificial Intelligence, 175(9-10):1498-1527, 2011. 26, 48

[DSdBC09] Karina Valdivia Delgado, Scott Sanner, Leliane Nunes de Barros e Fabio Gagliardi Cozman. Efficient solutions to factored MDPs with imprecise transition Probabilities. Em 19th ICAPS, Thessaloniki, Greece, 2009. 18

[FDdB14] Fernando L Fussuma, Karina Valdivia Delgado e Leliane Nunes de Barros. B ${ }^{2}$ RTDP: An efficient solution for Bounded-Parameter Markov Decision Process. Em Intelligent Systems (BRACIS), 2014 Brazilian Conference on, páginas 128-133. IEEE, 2014. 2, 47

[For10] Santo Fortunato. Community detection in graphs. Physics reports, 486(3):75-174, 2010. 52

[GLD00] Robert Givan, Sonia Leach e Thomas Dean. Bounded-parameter Markov decision processes. Artificial Intelligence, 122:71-109(39), 2000. xv, 1, 2, 18, 21, 22, 23, 24, $25,36,48$

[Har98] Darald J. Hartfiel. Markov set-chains. Lecture Notes in Mathematics; no 1695. Springer, 1998. 48

[How60] Ronald A. Howard. Dynamic Programming and Markov Process. The MIT Press, 1960. 6,16

[HSAHB99] Jesse Hoey, Robert St-Aubin, Alan Hu e Craig Boutilier. SPUDD: Stochastic planning using decision diagrams. Em Proceedings of the Fifteenth Conference on Uncertainty in Artificial Intelligence, páginas 279-288. Morgan Kaufmann, 1999. 12

[HZ01] Eric A. Hansen e Shlomo Zilberstein. LAO*: A heuristic search algorithm that finds solutions with loops. Artificial Intelligence, 129(1-2):35-62, 2001. 39, 40

[Iye05] Garud N Iyengar. Robust dynamic programming. Mathematics of Operations Research, 30(2):257-280, 2005. 48

[KCdC05] Daniel Kikuti, Fabio Gagliardi Cozman e Cassio Polpo de Campos. Partially ordered preferences in decision trees: computing strategies with imprecision in probabilities. Em IJCAI Workshop on Advances in Preference Handling, páginas 118-123, Edinburgh, United Kingdom, 2005. 15

[KMWG11] Andrey Kolobov, Mausam Mausam, Daniel Weld e Hector Geffner. Heuristic search for generalized stochastic shortest path mdps, 2011. 47

[KU02] Igor O Kozine e Lev V Utkin. Interval-valued finite markov chains. Reliable computing, 8(2):97-113, 2002. 48

[LDK95] Michael L. Littman, Thomas L. Dean e Leslie Pack Kaelbling. On the Complexity of Solving Markov Decision Problems. Em Proc. of the Eleventh International Conference on Uncertainty in Artificial Intelligence, páginas 394-402, 1995. 7

[MA93] Andrew W Moore e Christopher G Atkeson. Prioritized sweeping: Reinforcement learning with less data and less time. Machine learning, 13(1):103-130, 1993. 52 
[MDNdB16] Daniel A. M. Moreira, Karina Valdivia Delgado e Leliane Nunes de Barros. Robust probabilistic planning with ILAO. Applied Intelligence, páginas 1-11, 2016. 2, 47

[MK12a] Mausam e Andrey Kolobov. Planning with Markov Decision Processes: An AI Perspective. Synthesis Lectures on Artificial Intelligence and Machine Learning. Morgan \& Claypool Publishers, 2012. 3, 4, 6, 11

[MK12b] Mausam e Andrey Kolobov. Planning with Markov Decision Processes: An AI Perspective. Morgan \& Claypool Publishers, 2012. 6, 7, 47

[MMX12] Shie Mannor, Ofir Mebel e Huan Xu. Lightning does not strike twice: Robust MDPs with coupled uncertainty. Em ICML. icml.cc / Omnipress, 2012. 48

[NG05] Arnab Nilim e Laurent El Ghaoui. Robust control of Markov decision processes with uncertain transition matrices. Operations Research, 53(5):780-798, 2005. 48

[NL08] Yaodong Ni e Zhi-Qiang Liu. Bounded-parameter partially observable Markov decision processes. Em Proceedings of the Eighteenth International Conference on International Conference on Automated Planning and Scheduling, páginas 240-247, 2008. 49

[NL13] Yaodong Ni e Zhi-Qiang Liu. Policy iteration for bounded-parameter pomdps. Soft Computing, 17(4):537-548, 2013. 49

[PT87] Christos Papadimitriou e John N. Tsitsiklis. The complexity of markov decision processes. Math. Oper. Res., 12(3):441-450, 1987. 6, 10

[Put94a] Martin L. Puterman. Markov Decision Processes. Wiley Series in Probability and Mathematical Statistics. John Wiley and Sons, New York, 1994. 3, 6, 7

[Put94b] Martin L. Puterman. Markov Decision Processes: Discrete Stochastic Dynamic Programming. John Wiley \& Sons, Inc., New York, NY, USA, 1st edição, 1994. 1, 3, 5, 36

[RdBD19] Willy Arthur Silva Reis, Leliane Nunes de Barros e Karina Valdivia Delgado. Robust topological policy iteration for infinite horizon bounded markov decision processes. International Journal of Approximate Reasoning, 105:287 - 304, 2019. 34, 37, 39, 52

[RDdB16] Willy Arthur Silva Reis, Karina Valdivia Delgado e Leliane Nunes de Barros. Distributed and Asynchronous Policy Iteration for Bounded Parameter Markov Decision Processes. Em XIII Encontro Nacional de Inteligência Artificial e Computacional, 2016. $34,41,52$

[Sab01] Régis Sabbadin. Possibilistic markov decision processes. Engineering Applications of Artificial Intelligence, 14(3):287 - 300, 2001. Soft Computing for Planning and Scheduling. 49

[SBSR04] Andrew J. Schaefer, Matthew D. Bailey, Steven M. Shechter e Mark S. Roberts. Modeling Medical Treatment Using Markov Decision Processes, páginas 593-612. Springer US, Boston, MA, 2004. 41

[Sch15] Dimitri Scheftelowitsch. The complexity of uncertainty in markov decision processes. Em 2015 Proceedings of the Conference on Control and its Applications, páginas 303-310. SIAM, 2015. 16, 47

[SCT ${ }^{+}$07] Ricardo Shirota, Fabio Gagliardi Cozman, Felipe Werndl Trevizan, Cassio Polpo de Campos e Leliane Nunes de Barros. Multilinear and Integer Programming for Markov Decision Processes with Imprecise Probabilities. Em Proceedings ISIPTA, páginas 395-404, Prague,Czech Republic, 2007. 16, 17 
[SdBC09] Karina Valdivia Delgado Scott Sanner, Leliane Nunes de Barros e Fabio G Cozman. Efficient Solutions to Factored MDPs with Imprecise Transition Probabilities. Em 19th International Conference on Automated Planning and Schedulling - ICAPS 2009, páginas 98-105, 2009. 26

[SFL98] Régis Sabbadin, Hélène Fargier e Jérôme Lang. Towards qualitative approaches to multi-stage decision making. International Journal of Approximate Reasoning, 19(3):441 - 471, 1998. 49

[ک̌́ku06] Damjan Škulj. Finite discrete time markov chains with interval probabilities. Em Soft Methods for Integrated Uncertainty Modelling, páginas 299-306. Springer, 2006. 48

[SL70] Jay K. Satia e Roy E. Lave Jr. Markovian decision processes with uncertain transition probabilities. Operations Research, 21:728-740, 1970. 1, 13, 14, 16

[TB07] Ambuj Tewari e Peter L. Bartlett. Bounded parameter Markov decision processes with average reward criterion. Em Learning Theory, páginas 263-277, Berlin, Heidelberg, 2007. Springer Berlin Heidelberg. 47

[TCdB07a] Felipe W. Trevizan, Fabio G. Cozman e Leliane N. de Barros. Planning under risk and knightian uncertainty. Em Proceedings of International Joint Conferences on Artificial Intelligence, páginas 2023-2028, Hyderabad, India, 2007. 18, 19, 48

[TCdB07b] Felipe W. Trevizan, Fábio Gagliardi Cozman e Leliane Nunes de Barros. Planning under risk and knightian uncertainty. Em IJCAI 2007, Proceedings of the 20th International Joint Conference on Artificial Intelligence, Hyderabad, India, páginas 2023-2028, 2007. 41

[Tre06] Felipe Werndl Trevizan. Um modelo unificado para planejamento sob incerteza. Dissertação de Mestrado, IME-USP, 2006. Available in: http://www.teses.usp.br/teses/disponiveis/45/45134/tde-15022010-161012/ptbr.php. 48

[TT13] Vera Tilson e David A Tilson. Use of a Markov decision process model for treatment selection in an asymptomatic disease with consideration of risk sensitivity. SocioEconomic Planning Sciences, 47(3):172-182, 2013. 41

[Wal91] Peter Walley. Statistical reasoning with imprecise probabilities. Chapman and Hall, 1991. 49

[WB93] Ronald J Williams e L. C. Baird. Analysis of some incremental variants of policy iteration: First steps toward understanding actor-critic learning systems. Relatório técnico, NUCCS-93-11. College of Computer Science, Northeastern University, Boston, 1993. xv, 7, 8

[WED94] Chelsea C. White III e Hank K. El-Deib. Markov decision processes with imprecise transition probabilities. Operations Research, 42(4):739-749, 1994. 1, 13, 16

[WS05] David Wingate e Kevin D Seppi. Prioritization methods for accelerating MDP solvers. Journal of Machine Learning Research, 6(May):851-881, 2005. 7, 52

[XM12] Huan Xu e Shie Mannor. Distributionally robust Markov decision processes. Mathematics of Operations Research, 37(2):288-300, 2012. 48 